To appear in ApJ

\title{
The DiskMass Survey. II. Error Budget
}

\author{
Matthew A. Bershady, ${ }^{1}$ Marc A. W. Verheijen, ${ }^{2}$ Kyle B. Westfall,,${ }^{1,2,3}$ David R. Andersen, ${ }^{4}$ \\ Rob A. Swaters, ${ }^{5}$ and Thomas Martinsson ${ }^{2}$
}

\begin{abstract}
We present a performance analysis of the DiskMass Survey. The survey uses collisionless tracers in the form of disk stars to measure the surface-density of spiral disks, to provide an absolute calibration of the stellar mass-to-light ratio $\left(\Upsilon_{*}\right)$, and to yield robust estimates of the dark-matter halo density profile in the inner regions of galaxies. We find a disk inclination range of $25-35^{\circ}$ is optimal for our measurements, consistent with our survey design to select nearly face-on galaxies. Uncertainties in disk scale-heights are significant, but can be estimated from radial scale-lengths to $25 \%$ now, and more precisely in the future. We detail the spectroscopic analysis used to derive line-of-sight velocity dispersions, precise at low surface-brightness, and accurate in the presence of composite stellar populations. Our methods take full advantage of large-grasp integral-field spectroscopy and an extensive library of observed stars. We show that the baryon-to-total mass fraction $\left(\mathcal{F}_{\text {bar }}\right)$ is not a well-defined observational quantity because it is coupled to the halo mass model. This remains true even when the disk mass is known and spatially-extended rotation curves are available. In contrast, the fraction of the rotation speed supplied by the disk at 2.2 scale lengths (disk maximality) is a robust observational indicator of the baryonic disk contribution to the potential. We construct the error-budget for the key quantities: dynamical disk mass surface-density $\left(\Sigma_{\text {dyn }}\right)$, disk stellar mass-to-light
\end{abstract}

\footnotetext{
${ }^{1}$ University of Wisconsin, Department of Astronomy, 475 N. Charter St., Madison, WI 53706; mab@astro.wisc.edu

${ }^{2}$ University of Groningen, Kapteyn Astronomical Institute, Landleven 12, 9747 AD Groningen, Netherlands; verheyen@astro.rug.nl

${ }^{3}$ National Science Foundation (USA) International Research Fellow

${ }^{4}$ NRC Herzberg Institute of Astrophysics, 5071 W Saanich Road, Victoria, BC V9E 2E7

${ }^{5}$ University of Maryland, Dept. of Astronomy, College Park, MD 20742
} 
ratio $\left(\Upsilon_{*}^{\text {disk }}\right)$, and disk maximality $\left(\mathcal{F}_{*, \text { max }}^{\text {disk }} \equiv V_{*, \text { max }}^{\text {disk }} / V_{\mathrm{c}}\right)$. Random and systematic errors in these quantities for individual galaxies will be $\sim 25 \%$, while survey precision for sample quartiles are reduced to $10 \%$, largely devoid of systematic errors outside of distance uncertainties.

Subject headings: galaxies: kinematics and dynamics - galaxies: stellar content - galaxies: halos - galaxies: spiral - galaxies: formation - galaxies: evolution galaxies: structure - galaxies: fundamental parameters $(\mathrm{M} / \mathrm{L})$ - dark matter techniques: spectroscopic - methods: data analysis

\section{INTRODUCTION}

Paper I of this series (Bershady et al. 2010) presented the DiskMass Survey (DMS), a study designed to break the disk-halo degeneracy (van Albada et al. 1985), and bypass a major roadblock in testing galaxy formation models. Without an independent measurement

of the mass-to-light ratio of the stellar disk $\left(\Upsilon_{*}^{\text {disk }}\right)$, it is not possible to determine the structural properties of dark matter halos from rotation curve decompositions. The DMS is an effort to make a direct, and absolute kinematic measurement of the mass surface-density of intermediate-type spiral disks $\left(\Sigma_{\text {dyn }}\right)$, calibrate $\Upsilon_{*}$, and determine the density profiles of dark matter halos in these systems. In a nutshell, the question we aim to answer is this: How maximal are normal spiral disks? Specifically, we want to know how much of the observed disk rotation within the inner 2 to 2.5 disk radial scale-lengths is in response to the mass of the disk itself.

In this survey, the amplitude of the vertical motions of collisionless tracers in 46 galaxies are measured via integral-field spectroscopy (IFS) of the integrated star-light, in conjunction with a photometric estimate of the vertical scale height of these tracers. The former is measured as a velocity dispersion using absorption-lines in the stellar continuum sensitive to old but luminous disk stars, typically K giants. The latter is based on the correlation between disk oblateness and radial scale-length. An estimate of the disk-mass surface density then follows dimensionally from these length and velocity scales. Our program is inspired by the insights of van der Kruit \& Searle (1981) and Bahcall \& Casertano (1984), and the pioneering observations of van der Kruit \& Freeman (1984, 1986) and Bottema (1993). Similar surveys are also underway (Herrmann \& Ciardullo 2009) using different collisionless tracers, albeit with the same dynamical approach.

Galaxies in the DMS were selected, as described in Paper I, based on apparent size, inclination and visual (qualitative) morphology. Given these constraints, selection from 
the UGC (Nilson 1973) resulted in a sample with disk central surface-brightness in range $\mu_{0, R}=20.5 \pm 1.1 \mathrm{mag}$, comparable to Freeman's (1970) result (translated to the $\mathrm{R}$ band) of $\mu_{0, R}=20.65 \pm 0.3 \mathrm{mag}$ for what are commonly referred to as "normal" spiral disks. Galaxies in the DMS lie at distances of 15 to $200 \mathrm{Mpc}$, with morphological types mostly between $\mathrm{Sb}$ and Scd. The sample spans factors of 100 in $K$-band luminosity, 8 in blue-to-infrared color, and 10 in disk size and central surface-brightness. A posteriori, we find the sample almost entirely has rotation velocities $>120 \mathrm{~km} \mathrm{~s}^{-1}$. Several studies (e.g., Dalcanton et al. 2004; Yoachim \& Dalcanton 2006) have shown that disk properties above and below this rotation-speed have distinct structural properties, including different fractions of thick to thin disk components, as well as different dust-to-star vertical scale-heights. Faster rotators tend to have relatively smaller thick-to-thin disk luminosity ratios, and shorter dust scaleheights. While these and other differences within the spiral galaxy population preclude global dynamical conclusions about disks systems based on the DMS sample, this survey does target the heart of the spiral population: Galaxies in the DMS are typical of TullyFisher surveys, and well-sample the knee of the spiral luminosity function, in which most disk stars are contained. As such, the DMS provides a particularly relevant calibration of $\Upsilon_{*}$ in massive, star-forming systems in the nearby universe.

While computing $\Sigma_{\text {dyn }}$ from stellar velocity-dispersions and scale-heights is conceptually simple, the actual measurements are demanding and the analysis complex. To compute disk maximality (here, $\mathcal{F}_{* \text { max }}^{\text {disk }}$ ) we also need to measure the rotation speed $\left(V_{\text {rot }}\right)$, orthogonally projected to the vertical stellar motions $\left(\sigma_{z}\right)$ used to determine $\Sigma_{\text {dyn }}$. This orthogonality presents an observational quandry, requiring us to choose modest inclinations for measuring both $V_{\text {rot }}$ and $\sigma_{z}$. At such inclinations it is also a challenge to determine the inclination angle itself. Finally, to complete our scientific objectives, we also must determine $\Upsilon_{*}^{\text {disk }}$. This requires further measurement and analysis to correct $\Sigma_{\text {dyn }}$ for the atomic and molecular gas content of the disk, and to correct surface-photometry for dust extinction. Disk dark-matter, if it exists, we assume is distributed with a scale-height similar to old disk stars.

Accurate $\Upsilon_{*}$ values are critical for inferring the dark-halo profiles in the wide range of galactic systems required to trace the cosmic history of the stellar baryon fraction. Hence the calibration of $\Upsilon_{*}$ is of prime relevance for understanding galaxy structure and formation. Ideally $\Upsilon_{*}$ would be calibrated for stellar populations spanning as wide a range of properties (age, metallicity, abundance) as possible. While the DMS does not sample extreme populations likely found in giant ellipticals, low-mass dwarf irregulars and spheroidals, and low-surface-brightness disks, the DMS contains a well-defined sample spanning a wide range of properties. This is ample for testing the mass zero-point of stellar population synthesis models as well as trends with color and star-formation rate. We argued in Paper I that $\Upsilon_{*}$ should be known to $30 \%$ or better in order to make substantial progress in determining, e.g., 
the maximality of spiral disks.

The goals for this second paper in the DMS series are to estimate, and present an accounting of, the error-budget of the primary derived quantities: $\Sigma_{\text {dyn }}, \Upsilon_{*}^{\text {disk }}$ and $\mathcal{F}_{* \text { max }}^{\text {disk }}$. Specifically, we verify we can reach the stated goal of $30 \%$ uncertainty in $\Upsilon_{*}$. As a secondary goal we substantiate our assertion that low-inclination disks are optimal for this type of measurement. To achieve these goals we step through every major aspect of our measurement and analysis, starting with considerations that informed our survey strategy, namely, uncertainties in the disk stellar velocity ellipsoid (SVE; §2.1), disk oblateness (§2.2), and inclination (§2.3). Within the methodological framework established in Paper I, we detail all facets required to arrive at an accurate and reliable estimate for $\sigma_{z}(\S 3)$. We focus here on the challenging aspects of the spectral analysis unique and central to the survey. Distance errors are considered briefly in $\S 4$. The development in these preliminary sections allows us to establish our expected error budget for the primary derived quantities from our survey $(\S 5): \Sigma_{\text {dyn }}, \Upsilon_{*}^{\text {disk }}$, and $\mathcal{F}_{* \text { max }}^{\text {disk }}$. The error-budget analysis is summarized in $\S 6$. In Paper III (Westfall et al. 2010, in preparation) we present the cross-correlation technique used to derive the SVE from pilot observations of UGC 6918. The same galaxy is used here to illustrate central features of our analysis. All distant-dependent quantities are scaled to $\mathrm{H}_{0}$ $=73 \mathrm{~km} \mathrm{~s}^{-1} \mathrm{Mpc}^{-1}$. Logarithmic errors are specified as $\Delta \ln X \equiv \epsilon(X) / X$, where $\epsilon(X)$ is the error in quantity $X$.

\section{SURVEY DESIGN OPTIMIZATION}

We argued in Paper I that a nearly face-on approach to measuring both the disk mass surface-density and the total mass is optimal because of the quadratic dependence of $\Sigma_{\text {dyn }}$ on $\sigma_{z}$, compared to the linear dependence on the disk scale-height. We develop this argument by comparing ( $\S 2.1$ and $\S 2.2)$ the constraints available on the shape of the SVE compared to those on the disk oblateness. We quantify in $§ 2.2$ the contribution of disk-oblateness uncertainties to our error budget. Because of the uncertainties in the SVE there is a detailed balancing that can be done between the amount of projection of the vertical versus tangential motions of the stars into the observers line-of-sight. We tie together the uncertainties in the SVE and inclination in $\S 2.3$ to arrive at an optimized disk inclination range for the DMS. 


\subsection{Disk Stellar Velocity Ellipsoid}

The SVE is described by its radial, tangential and vertical components: $\sigma_{R}, \sigma_{\theta}$, and $\sigma_{z}$. Here we do not entertain the effects of a tilted ellipsoid. What is relevant for the DMS, in order to deproject $\sigma_{z}$ from the observed line-of-sight velocity dispersion, is the shape of the SVE. This shape is conveniently parameterized by the axial ratios $\alpha \equiv \sigma_{z} / \sigma_{R}$ and $\beta \equiv \sigma_{\theta} / \sigma_{R}$. Expectations from the Solar Neighborhood are that $\sigma_{R}>\sigma_{\theta}>\sigma_{z}$, specifically with $0.5<\alpha<0.6$ and $0.6<\beta<0.7$ for the thin disk, depending on what tracers are used (Binney \& Merrifield, 1998). However, little is actually known about these values in external galaxies, and no measurement exists to indicate if there is a radial dependence to these ratios. Radial dependencies are likely, based on dynamical arguments, including the simple observation that galaxies tend to be dynamically hotter in their interior.

Extant knowledge of $\alpha$ and $\beta$ for external spiral galaxies (summarized in Shapiro et al. 2003) consists of integrals over major- and minor-axis kinematic data within the inner 1-3 radial scale-lengths of 6 moderately inclined galaxies, requiring assumptions regarding the form and validity of the epicycle approximation and asymmetric drift equation; and 40 edge-on galaxies, requiring further dynamical assumptions and scaling arguments (van der Kruit \& de Grijs 1999). All measured values of $\alpha$ and $\beta$ for external galaxies are global quantities.

These external-galaxy studies have used the epicycle approximation to measure $\beta$, namely: $\beta=\frac{1}{2}\left[\partial\left(\ln V_{\theta}\right) / \partial(\ln R)+1\right]$, where $V_{\theta}$ is the tangential speed of the stars (see Westfall 2009). Data presented in Shapiro et al. (2003) imply $0.6<\beta<0.8$. Since $\beta$ depends on the derivative of the tangential speed, in general we do not expect $\beta$ to be constant with radius. To obtain global values for $\beta$ these same studies have parameterized the radial dependence of the tangential speed as a power-law. A parameterization serves to minimize errors associated with the derivative in the epicycle approximation. Adopting a more realistic functional form for the tangential speed (e.g., the "universal rotation curve" of Courteau, 1997) would be preferable. With a suitable model for the tangential speed, the uncertainty in the value of $\beta$ is not of concern because the very stellar measurements needed to determine the line-of-sight velocity dispersion $\left(\sigma_{\mathrm{LOS}}\right)$ can be used to estimate $\beta$.

To derive $\sigma_{z}$ from $\sigma_{\mathrm{LOS}}$ then is largely a matter of determining $\alpha$, and herein lies the

problem. From existing data there is some hint of a trend in the ellipsoid ratio $\alpha$ to larger values (0.8) for types earlier than $\mathrm{Sb}$, but this is based on a few points with substantial errors. It is conceivable this trend is due to increasing bulge contamination in earlier types. For later types, the mean ratio falls in the range $0.5<\alpha<0.7$, with less indication of trend, but a $50 \%$ spread $(1 \sigma)$, i.e., $0.3<\alpha<0.9$. For an edge-on approach to measuring disk mass, this translates into a $100 \%$ systematic error in $\Sigma_{\text {dyn }}$ from the velocity component alone. In 
short, the SVE in disk galaxies is not well-known.

This brief discussion concludes that the experimental design to measure disk mass via the stellar $\sigma_{\text {LOS }}$ must either determine the SVE shape very well, or choose an inclination range where $\sigma_{z}$ is favorably projected and uncertainties in the SVE lead to little error in the correction. It turns out that a nearly face-on inclination answers both desiderata. Future papers in this series will show the dependence on inclination for the SVE determination (see also Westfall 2009). Here we provide estimates for typical $\alpha$ and $\beta$ values and uncertainties in our survey in $\S 3.5 .4$.

\subsection{Disk Oblateness}

We show it is possible to estimate $z_{0}$, the vertical scale-height used to determine $\Sigma_{\text {dyn }}$ (equation 1 of Paper I), reliably from $h_{R}$, the exponential radial disk scale-length. Several independent, photometric surveys exist of edge-on galaxies, linking scale-height and scalelength to galaxy type, rotation speed, and other readily observable global properties. In contrast to the situation for the SVE, a relatively clear picture emerges.

\subsubsection{Disk vertical structure}

To place disk oblateness properly into the context of $\Sigma_{\text {dyn }}$ error budget, it is necessary to clarify the definition of scale-height in terms of the vertical structure of a disk. Anticipating the generalization in $\S 5$ we begin by noting the formulation for $\Sigma_{\text {dyn }}$ in equation (1) of Paper I assumes a locally isothermal disk with a vertical mass-density distribution function of $\operatorname{sech}^{2}\left(z / z_{0}\right)$. Other density distributions are also appropriate, including $\operatorname{sech}\left(z / z_{\text {sech }}\right)$ and $\exp \left(-z / h_{z}\right)$ (van der Kruit 1988). A more general expression of $\Sigma_{\text {dyn }}$ can be written to include the three vertical distribution functions: $\Sigma_{\text {dyn }}=\sigma_{z}^{2} / \pi k \mathrm{G} h_{z}$, where $k=3 / 2,1.7051$, 2 for exponential, sech, or $\operatorname{sech}^{2}$ vertical mass distributions. Since all three functions behave as exponential distributions at large scale-heights, we can relate $z_{0}=\sqrt{2} z_{\text {sech }}=2 h_{z}$ in our specific functional formulation. We use these equalities throughout the discussion here, and define oblateness as $q \equiv h_{R} / h_{z} \equiv 2 h_{R} / z_{0} \equiv \sqrt{2} h_{R} / z_{\text {sech }}$.

Unfortunately, the issue of what is the actual vertical mass-density distribution of disks remains outstanding. In the DMS, we parameterize this ignorance in the possible range of $k$. An isothermal distribution is conceptually preferable given a simple picture of a single, relaxed population of disk stars. However, the non-isothermal density distributions, which

have discontinuous potentials at the mid-plane, appear to be a suitable approximation to 
a combination of a thin and thick stellar disk plus a very thin mid-plane distribution of atomic and molecular gas and very young stars. This is a model qualitatively consistent with our picture of the Milky Way. Indeed, recent studies of resolved stellar populations in nearby, edge-on-galaxies also show such three-component stellar systems, with scale-heights increasing with population age (Seth et al. 2005). Observations in the near-infrared, capable of penetrating the disk mid-plane dust layer, indicate that an exponential vertical distribution is likely the best functional form (Wainscoat et al. 1989, Aoki et al. 1991, de Grijs \& van der Kruit 1997), although Aoki et al. point out that the steepness of the density distribution inferred from the $K$-band light near the mid-plane that they observed in NGC 891 could be due to an excess of red super-giants with low M/L. Yet even if the light near the galaxy mid-plane is dominated by massive stars with low $\Upsilon$, the additional gas components still make an exponential vertical mass-density distribution a plausible approximation.

Luckily, the uncertainties in the vertical mass-density distribution can be decoupled from uncertainties in estimating a characteristic scale-height, $h_{z}$, for the purposes of measuring $\Sigma_{\text {dyn. }}$. This is true so long as the light-weighting of the kinematic signal in face-on galaxies is the same as what defines the photometric vertical profile in edge-on samples. This is a reasonable assumption when coupling the near-infrared light distribution in edge-on systems to gravity-insensitive kinematic signal dominated by cool stars in face-on samples. (The lines in our spectroscopic regions of $\mathrm{Mg} \mathrm{Ib}$ and Ca II meet these desiderata.) In this situation the relative contribution of different disk components (e.g., thin and thick) is statistically self-consistent in photometric estimates of scale-heights (observed in edge-on samples) and in kinematic estimates of $\sigma_{\mathrm{LOS}}$ (observed in face-on galaxies); $k$ parameterizes the dynamical variation in the disk vertical mass-density to the estimate of $\Sigma_{\text {dyn }}$. Accordingly, we focus now on what we know about the correlation of the vertical to the radial scale-lengths of the disk light distribution, and then explore how the correlation might be biased by wavelength or the presence of multiple disk components.

\subsubsection{A fiducial relationship}

Figure 1 illustrates our compilation of four studies (Kregel et al. 2002; Pohlen et al. 2000; Schwarzkopf \& Dettmar 2000; and Xilouris et al. 1997, 1999) of the vertical to radial disk scale-length ( $h_{z}$ and $h_{R}$, respectively) based on photometry of edge-on galaxies. All absolute values have been rescaled consistently to our choice of $\mathrm{H}_{0}$. For contrast and application to the DMS, the two panels break out the spirals into intermediate-types (top) and early and late types (bottom). The majority of DMS galaxies in the Phase-B sample (Paper I), for which there are stellar spectroscopic observations, have morphological types 
consistent with those in the top panel.

For the Kregel. et al. (2002) sample, we have adopted their I-band results as they recommend, included 3 more-nearby galaxies from their later work (NGC 891, 5170, and 5529; Kregel et al. 2004), but excluded ESO555-G36 because of contamination due to a bright fore-ground star (de Grijs 1998).

Of the seven edge-on galaxies we include in Figure 1 from Xilouris et al. $(1997,1999)$, two overlap with the Kregel et al. sample; however, these galaxies have been observed independently and subjected to different modeling techniques. The Xilouris et al. observations include in $B, V, I, J$, and $K$ bands. Since we are interested primarily in the distribution of old, luminous stars also used as dynamical tracers, and to be consistent with the Kregel et al sample we therefore focus on the red and near-infrared bands (including $I$ ). Plotted are the $I$-band values, after adjusting their distance-estimates based on recession velocities corrected for Virgo in-fall, consistent with the procedures in Kregel et al. (2002).

Accordingly, for the Schwarzkopf \& Dettmar (2000) sample, for which they have observed in $r, R, H, K$ bands, we have restricted their sample to those 15 galaxies with nearinfrared $H$ - or $K$-band measurements. Similarly, for the Pohlen et al. (2000) sample, for which they have observed in $g, r / R, i$ bands, we have restricted their sample to only those 5 including $i$-band measurements. We find the scatter increases substantially if we include bluer measurements. These last two studies are particularly interesting because unlike the two previous studies which use only an exponential vertical distribution, they allow the vertical distribution to be characterized by either exponential, sech, or sech ${ }^{2}$ functions. Their tabulated values represent the corresponding effective exponential scale-heights regardless of the fitted functional form. By limiting their sample to just the near-infrared measurements, the trends of radial to vertical scale-lengths appear identical for all functional forms.

While the scatter in Figure 1 may at first glance look large, there are two significant degrees of coherence. First, disk oblateness for intermediate-types Sb-Scd correlates with the scale of the disk ( $h_{R}$; top panel). Second, there is an offset in the relation with galaxy types earlier than $\mathrm{Sb}$ or later than Scd (top versus bottom panels). The correlations are in the sense that later-type galaxies (less bulge-dominated systems) have thinner disks, and larger disks (at a given bulge-dominance) are thinner. This makes some astrophysical sense in that whatever produces a bulge or pseudo-bulge either represents a merging or disk instability process that would heat the disk. The fact that disk thickness does not scale linearly with disk scale-length must reflect a more complicated interplay between angular momentum and the mechanisms responsible for disk heating. While previous studies have noted the typedependence, the correlation with scale appears as, if not more, fundamental, and is likely associated with the general scale-dependence of galaxy properties (e.g., van den Bergh 1960). 
Key here for minimizing scatter is this at least bivariate correlation.

Our fit to the Sb-Scd galaxies typical of the DMS for the Kregel et al. subset (shown in Figure 1) statistically matches both the slope, zero-point and scatter of the other three samples; these four samples are statistically indistinguishable in this regard. The Kregel et al. sample is a good match to our own both in the distribution of physical size, surfacebrightness, rotation velocity, and morphological type (compare Table 1 of Kregel et al. [2002] with our Table 3 of Paper I.) Hence we adopt this fit as the operational relation for the DMS at this time:

$$
\log \left(q_{R}\right) \equiv \log \left(h_{R} / h_{z}\right)=0.367 \log \left(h_{R} / \mathrm{kpc}\right)+0.708 \pm 0.095
$$

This is consistent with $h_{z} \propto h_{R}^{2 / 3}$. We conclude that the effective oblateness of the disk can be reasonably estimated to about $25 \%$ ( $1 \sigma$ systematic error for any one galaxy) for face-on galaxies typical of the DMS sample, simply via measurement of $h_{R}$ in the $I$-band. We do not distinguish between sub-types for Sb-Scd since there is presently no solid statistical basis (e.g., K-S test) to do so. For error-budgeting purposes we adopt the logarithmic derivatives $\Delta \ln q_{R}=0.25$ and $\Delta \ln h_{R}=0.03$, the latter following the analysis of MacArthur et al. (2003). We believe $\Delta \ln q_{R}=0.25$ is likely an over-estimate for reasons given in $\S 2.2 .3$ and $\S 2.2 .5$. For the 3 galaxies in the DMS earlier than $\mathrm{Sb}$ and the 2 galaxies later than $\mathrm{Sd}$, we use the data points in the bottom panel of Figure 1 to estimate offsets from the above fiducial, assuming the same slope. This adds $15 \%$ additional uncertainty in $q_{R}$ (a total of $29 \%$ instead of $25 \%$ uncertainty in the oblateness correction), but only for $\sim 10 \%$ of the DMS sample. We expect a more comprehensive compilation of the literature or future analysis will add to, and improve, the calibration of $q_{R}$ for all types.

\subsubsection{Wavelength dependence}

While we have calibrated $q_{R}$ in the $I$-band, we also explored the wavelength dependence of this relationship. This is important because we know disks in external galaxies have radial color gradients presumably due to changes in mean age and metallicity with radius; expectations from the solar neighborhood are that vertical color gradients should exist as well due to increasing scale-heights with stellar population age. A priori, it is unclear if the vertical and radial gradients scale such that disk oblateness appears constant with wavelength. If not, in order to avoid systematics in deriving $\Sigma_{\text {dyn }}$ the oblateness-radial scale-length relation must be calibrated at the same wavelength used to measure radial scale-length, and at a wavelength appropriate to measure the scale-height of the kinematic tracers. 
Inspection of the sizes $\left(h_{R}\right)$ and oblateness $(q)$ as a function of band-pass in the Xilouris et al. sample shows clearly that size increases while oblateness increases significantly at shorter wavelengths. Larger radial scale-lengths in the blue are not unexpected: galaxy disks have color gradients in the sense that they become bluer with radius, e.g., see de Jong (1996a) in the face-on context. In fact, an inspection of the radial scale-lengths tabulated by de Jong (1996b) in $B$ and $K$ bands shows just this effect. Likewise a decrease of $q$ in blue light is consistent with expectations that star-formation is concentrated toward the mid-plane, despite the impact of extinction and scattering on the apparent light distribution. While the Xilouris et al. data shows an effect from $I$ to $B$ bands, there is also some hint of an effect between $I$ and $V$ as well, but there is insufficient data to probe if differences exist between $I$ and redder bands.

Yoachim \& Dalcanton (2006; hereafter YD) find a similar result in a larger sample of 34 edge-on galaxies observed in $B, R, K$ bands. However, they also see a decrease in $h_{z}$ in the $K$-band, which they tentatively interpret as a combination of extinction, observational depth, and stellar-population effects. This data set is rewarding to work with because, like the work of Kregel et al., the authors have published their uncertainties. We have plotted their measurements in Figure 2 (top panels) for all of their band-passes. The histogram to the right shows the residuals about the relationship given by equation (1). From these figures we draw two conclusions.

First, our calibration with independent data provides an excellent description of the data. The mean residuals in the $R$ and $K$ bands are under $4 \%$, and the scatter is somewhat lower $(0.07 \mathrm{dex}$, or $18 \%)$. If we assume their sample is similar to what we have compiled from the above studies, this indicates that our zero-point for $q_{R}$ is likely accurate to better than $7 \%$ in the red and near-infrared, and that our estimate of $\Delta \ln q_{R}$ can be reduced. Because we cannot verify this assumption, we retain $\Delta \ln q_{R}=0.25$.

Second, the mean residual in the $B$-band differ by at most $0.05 \operatorname{dex}(12 \%)$, and while the $R$ and $K$ band mean residuals differ formally by $5 \%$ they are indistinguishable on the basis of a K-S test. This result requires no assumptions because it is a differential comparison. In other words, our finding is that while oblateness and scale-length change with wavelength, to first order this simply shifts galaxies along the regression given by equation (1). This implies that $q_{R}$ can be readily estimated via any accurate scale-length measurement from the blue to near-infrared wavelengths. Since we are interested in the scale-height of the old stars, this still requires scale-length measurements in the red or near-infrared. 


\subsubsection{Thick disk component}

The YD data set can also be used to explore what impact a thick-disk component has on our relation for $q_{R}$. This is important because the presence of a significant thick disk, if hidden due to lack of projection in a face-on system, would systematically increase $\sigma_{\text {LOS }}$ relative to a system dominated by a thin disk. Unless the effective oblateness, $q_{R}$, accounts for this thick component, the result would be an overestimate of $\Sigma_{\text {dyn }}$.

Ever since the early work of van der Kruit \& Searle (1981), there have been on-going searches for thick disks around external spiral galaxies. For example, with the advent of deep CCD photometry, Morrison et al. (1994) found NGC 5097 was absent a Milky Way-like thick disk, with at most a $2 \%$ contribution (by light) from a thicker component. Fry et al. (1999) found no evidence for a thick disk in NGC 4244 also down to very faint light levels. However, even the earlier photographic work of van der Kruit \& Searle (1981) sometimes showed small departures from a single exponential vertical light distribution at very low light levels. The work of YD convincingly shows the need for more than a single component in a wide range of late-type spirals, at least qualitatively consistent with the work of Seth et al. (2005). The impact of the YD two-component disk fits in the $R$ band are shown in the bottom panel of Figure 2. Compared to the one-component fits, the oblateness of the two-component thin plus thick disk increases $36 \%$ for the thin disk and decreases $50 \%$ for the thick disk. If the kinematic signal in face-on systems could be uniquely identified with thin or thick components, then these data could be used to recalibrate equation (1). The scatter in the $q_{R}$ relation for the thin-disk component is comparable to that for the Kregel et al. sample $(25 \%)$, while for the thick-disk component the scatter is only slightly larger $(32 \%)$.

For the DMS, however, we expect both thin and thick disks to contribute to our kinematic signal. For example, YD find the ratio of thick to thin scale-heights is roughly a factor of 2.4. This is much larger than the value of 1.1 Seth et al. (2005) found for the ratio of scale-heights for red giant-branch (RGB) to asymptotic giant-branch (AGB) stars in similar galaxies. Hence YD's findings are likely not coupled to population-age effects, but some other mechanism. In other words, it is plausible to assume that RGB stars (our expected primary kinematic tracer) are well mixed in both thin and thick components as parameterized by YD. In this case, while superficially the changes in disk oblateness going from one to two-disk components appear alarmingly large, we show the single-disk fits are a suitable characterization of the vertical light-distribution for mass-modeling.

A rough assessment of the importance of the thick disk to the integrated light can be gleaned from YD's Figure 7: The observed excess light departing from a single-disk fit becomes appreciable above $\sim 3 h_{z}$. The excess accounts for no more than about $5 \%$ of the total light enclosed within their measuring window in scale-height (their fitting region is 
$R / h_{R}<4$ and $z / h_{z}=6$ to 8 ), but the excess is at large heights where the dynamical impact in terms of the effective scale-height is more significant. This percentage appears fairly independent of rotation velocity. On the other hand, YD show that the luminosity ratio of thick to thin disks decreases substantially at higher rotation speeds, while the ratio of vertical scale-heights is constant. (This requires the thick disk radial scale-length to increase at lower circular velocities relative to the thin disk, which is what they see.) For $V_{\text {rot }}>100 \mathrm{~km} \mathrm{~s}^{-1}$, the contribution of the thick-disk to the overall disk surface-brightness is below $10 \%$ in the $R$ band. This is consistent with previous studies finding little thick-disk contributions or evidence for disk-flaring within 4 radial scale-lengths (equivalent to larger thick-disk scalelengths). For example, NGC 5097 has a rotation speed of $220 \mathrm{~km} \mathrm{~s}^{-1}$ (Casertano 1983), while NGC 4244 has a rotation speed of $100 \mathrm{~km} \mathrm{~s}^{-1}$ (Olling 1996). For the DMS, $92 \%$ of the sample has $V_{\text {rot }}>120 \mathrm{~km} \mathrm{~s}^{-1}$. This is in a regime where YD estimate the thick-disk component is below $10 \%$ of the total disk light contribution and thick and thin disk radial scale-lengths are equivalent. (Only $4 \%$ of our sample would be expected to have $>20 \%$ thick-disk contributions based on their calibration.) Therefore we anticipate the single-disk fits provide an fairly accurate characterization of disk oblateness for the DMS.

We refine this initial assessment by calculating the first moment of the vertical light profile $1 z_{1}$, for the one- and two-disk model parameters in YD's Tables 3 and 4. This moment is a non-parametric proxy for $h_{z}$, sensitive to the shape and extent of the light distribution. For single-component $\operatorname{sech}^{2}$ vertical light distribution $z_{1} / h_{z}=2 \ln 2$. Because YD provide the median parameters over a set of different fitting schemes, where the median is taken for each parameter individually, these values do no make self-consistent sets. For example, if one integrates the thin and thick disk profiles specified in their Table 4, their tabulated ratio of thick to thin disk luminosity is not recovered. We proceed by adopting the median scale-lengths and scale-heights, but renormalize the central surface-brightness of the thin and thick disks to simultaneously yield (i) the tabulated thick-to-thin luminosity ratio and (ii) the same vertical surface-brightness profile in the mean for single and two-disk models in the region for $z<3 h_{z}$ and $R / h_{R}<4$. The latter essentially reproduces their Figure 7. We also implement their correction for internal extinction for the 2-disk model, which in their scheme is a correction to the thin-disk luminosity only, but still require condition (ii) to be met.

The difference between the face-on, radial surface-brightness profiles for the one- and

\footnotetext{
${ }^{1}$ This is the moment at a specific radial and azimuthal location $(R, \theta)$ in the disk, i.e., not the projected edge-on light distribution. In this case, $z_{1}(R, \theta)=\int_{0}^{\infty} I(R, z, \theta) z d z / \int_{0}^{\infty} I(R, z, \theta) d z$, where $I(R, z, \theta)=$ $\sum_{1, \ldots, j} I_{j}(R, z, \theta)$, and $I_{j}(R, z, \theta)=I_{0, j} e^{-R / h_{R, j}} \operatorname{sech}^{2}\left(z / z_{0, j}\right)$ is the light density for a single isothermal component, $j$.
} 
two-disk models, renormalized as outlined above, are shown in the top panel of Figure 3. Without extinction, the differences are of order 5\%, as anticipated, increasing at larger radii. The effect of extinction is to decrease the contribution from the thin-disk component; with our renormalization this brings the one- and two-disk face-on surface-brightness profiles into closer agreement. The ratio of vertical first moments in the bottom panels of Figure 3 show significant scatter consistent with measurement errors, and a trend to relatively smaller $z_{1}$ for the one-disk models at slower rotation speeds. In the regime of interest to the DMS between $1<R / h_{R}<3$ for the fast-rotating disks $\left(V_{\text {rot }}>120 \mathrm{~km} \mathrm{~s}^{-1}\right.$ ), the $z_{1}$ ratio is consistent with unity; with no extinction the weighted mean ratio is 0.94 , and with extinction this increases to 1.06. In the context of the concerns framed at the beginning of this subsection, it is clear that the presence of a thick-disk component has an insignificant impact on the effective disk scale-height relevant for disk-mass measurement.

In Figure 4 we show there is a correlation between offsets from the fiducial oblateness relation of the thick and thin components, as well as between the one- and two-disk models. For the later, the two-disk offsets are computed as the luminosity weighted mean offset of the thick and thin components. In other words, when the thick disk tends to be more or less oblate than what would be inferred by its radial scale-length, so too does the thin disk component; together these offsets are in lock-step with the oblateness variation of the one-disk model. Whether due to astrophysics or the fitting process, both models represent the same departures from the fiducial oblateness relation. This result combined with the insensitivity of $z_{1}$ to one- versus two-component disk fits leads us to conclude that the singledisk fits provide a statistically accurate characterization of disk oblateness for the DMS.

\subsubsection{Additional correlations and concerns}

It is reasonable to expect that the estimation of $h_{R} / h_{z}$ will improve in the near future. Measurement precision will inrease with deeper images, sample sizes will increase with the extensive imaging surveys now in hand, and sample homogeneity will increase with better classification. In this context, it is relevant to recall that the single-disk fits of YD yield $30 \%$ less scatter than our calibrators.

It is also likely that the estimation of $h_{R} / h_{z}$ will improve because of additional correlations between disk oblateness and other observables. For example, Dalcanton et al. (2004), Kregel et al. (2005) and YD show there is a correlation between disk rotation speed and $h_{R} / h_{z}$. Kregel et al.'s sample also shows a possible correlation of $h_{R} / h_{z}$ with H I mass. Size, rotation-speed, and mass are all proxies for galaxy scale (in the van den Bergh sense), so these correlations may not be independent. Indeed, we do not see any correlations in the 
residuals from $q_{R}$ with these other quantities. However, a principal-component analysis may further reduce the scatter in Figure 1. The significant residuals from the correlation of $h_{R} / h_{z}$ with $h_{R}$ (in excess of observational error) indicates a further reduction in scatter of $q_{R}$ may be possible.

One other relation that is being used in the literature to estimate $h_{z}$ (Herrmann \& Ciardullo 2009) is an apparent correlation of disk oblateness with surface-brightness (Bizyaev \& Mitronova 2002, 2009). Taken at face value, the formula provided by Bizyaev \& Mitronova (2002) produces estimates of $h_{z}$ based on measurements of $h_{R}$ and the disk central surfacebrightness (in the $K$ band) with $22 \%$ precision for their sample. Applying equation (1) to their sample we find $h_{z}$ is estimated only to 30 to $40 \%$ precision, depending on what subset of their data is used. The subset with larger scatter is their preferred subset of larger galaxies, for which they believe their measurements are more robust. Somewhat worrisome is the fact that this same sample has an offset of about $20 \%$ in the mean from equation (1) as well. Furthermore, the formulae relating $h_{R} / h_{z}$ to $K$-band central disk surface-brightness from their two studies (2002 versus 2009) do not give consistent results, with $h_{R} / h_{z}$ differing by over $60 \%$ near the Freeman value (assuming typical $B-K$ colors for disks). The sample and photometric data are similar between these two studies by the same authors, but there is insufficient information to determine why their derived relation has changed. More puzzling is the fact that we do not find a convincing correlation in the data sets we have analyzed here (see for example Kregel et al. 2005). For these reasons equation (1) remains our estimator for disk oblateness, and we would caution against using surface-brightness as a proxy for disk oblateness at this time.

\subsubsection{Contributions to the error budget}

The uncertainties in estimating $h_{z}$ from applying the calibration of $h_{R} / h_{z}$ in equation (1) have both systematic and random components. Random errors in $h_{z}$ arise from the propagation of random errors in measuring $h_{R}$ in an individual galaxy. There are also likely to be stochastic, astrophysical variations in disk oblateness from galaxy to galaxy of a given size and type. These variations contribute, no doubt, to some of the scatter about the mean relation seen in Figure 1 even for the restricted subset of Sb-Scd systems. However, while such variation leads to systematic errors in estimating $h_{z}$ for individual galaxies, the effect is random for the sample as a whole. Hence errors in $h_{z}$ that arise from real variation in disk oblateness will be substantially reduced in a statistical sense for the survey as a whole,

and will be random errors. The propagation of these errors for $\Sigma_{\mathrm{dyn}}, \Upsilon_{*}^{\text {disk }}$ and $\mathcal{M}_{\mathrm{dyn}}^{\text {halo }}$ are discussed in $\S 5$, where we also consider the impact of a thick disk. 


\subsection{Disk Inclination}

How close to a face-on orientation is best? Low inclinations minimize (a) line-of-sight contamination to $\sigma_{z}$ from $\sigma_{R}$ and $\sigma_{\theta}$, (b) beam-smearing from velocity-field shear, and (c) extinction effects on the derived surface-brightness, color and velocity dispersion of the disk. However, in order to obtain the full mass-budget of the galaxy, we must measure not only $\Sigma_{\text {dyn }}$ but the total mass $\left(\mathcal{M}_{\text {dyn }}^{\text {tot }}\right)$ via measurements of the projected circular rotation and disk inclination $(i)$.

Nominally, we would prefer galaxies which are minimally inclined to derive kinematic inclinations from their velocity fields, with sufficient accuracy such that errors on the derived total masses from rotation curves are comparable to those of the disk mass surface-density from $\sigma_{z}$. However, the detailed trade-off depends on the specific science goal. Within our survey there are two related ones, namely (i) measurement of $\Sigma_{\text {dyn }}$ and calibration of $\Upsilon_{*}^{\text {disk }}$, where low inclination is preferred $\left(<30^{\circ}\right)$ to minimize line-of-sight contamination to $\sigma_{z}$; and (ii) measurement of disk-to-total mass ratios (from rotation-curve decompositions), which favors modest inclination ( $25^{\circ}$ to $45^{\circ}$ ) to balance disk- and total-mass errors. The $\Sigma_{\text {dyn }}$ errorbudget depends on inclination primarily through the correction to $\sigma_{\mathrm{LOS}}$ for the projected $\sigma_{R}$ and $\sigma_{\theta}$ components of the SVE. The degradation in precision of measuring $\sigma_{z}$ is shallower than a simple $1 / \cos i$ function because the SVE shape can be measured directly from the data, and this measurement improves with increasing inclination (up to moderate inclinations). Westfall (2009) addresses the optimum inclination for SVE decomposition. The following discussion encapsulates the arguments used to arrive at these quantitative inclination ranges, and how we optimized our selection.

Our base-line approach has been to rely on kinematic estimates of inclination (Paper I), which we find to be superior at low inclination to photometric estimates based on apparent disk ellipticity (Andersen \& Bershady 2003). Indeed, one of the motivations of Andersen's (2001) survey was to establish the efficacy of using $\mathrm{H} \alpha$ velocity fields measured with coarselysampled IFUs to constrain disk inclination in preparation for the current DMS. Because of the high $\mathrm{S} / \mathrm{N}$ and spectral resolution of the $\mathrm{H} \alpha$ data, we are able to determine kinematic inclinations with a precision better than 2 degrees at the relatively low inclination of 28 degrees (see Appendix A).

As noted in Paper I, inclination can also be estimated by inverting the Tully-Fisher relation (TF; Tully \& Fisher 1977), with the advantage that random errors in so-called inverse-Tully-Fisher (iTF) inclinations do not blow up at low inclination, as do both photometric ellipticity and kinematic isovelocity methods. For nearly face-on galaxies the iTFinclination random errors in percentage terms are simply proportional to the quadrature sum of the projected velocity and luminosity errors (the latter including distance uncer- 
tainties), while the systematic errors are proportional to the scatter in the TF relationship. Because of the potential for large systematic errors (i.e., a priori: What is the TF scatter for the sample or source in hand, and how accurate are the non-Hubble flow corrections to the observed systemic velocity?), our preference is to work in a regime of inclination where kinematic inclinations can be measured with small enough errors to reduce the overall error budget (random and systematic), and where we can directly verify a galaxy's location on the Tully-Fisher relation. While our survey sample was selected accordingly, post-facto, highprecision kinematic inclinations are not obtainable for all survey galaxies. Consequently we have also utlized additional inclination constraints from iTF in some cases (Andersen et al. in preparation).

Figure 5 illustrates the trade-offs with inclination between logarthmic errors in totalmass and disk mass surface-density, accounting for errors in inclination only (formulae are given in the Appendices as noted). Left-hand panels show errors assuming inclination is measured from fitting the $\mathrm{H} \alpha$ velocity fields with a model of an inclined, rotating disk (Appendix A.1). In the top panel, data points represent a preliminary analysis of 70 galaxies in the DMS, yielding quantitative expectations for errors introduced by inclination uncertainties (Andersen 2001). Black points and curves (decreasing left to right) represent total-mass errors $\left(\Delta \ln \mathcal{M}_{\text {dyn }}^{\text {tot }}\right.$, Appendix B.3), with dashed lines enclosing the range of galaxies with good velocity fields and regular kinematics, and the solid curve representing the mid-point of this distribution. The dotted curve contains the most deviant points with the largest kinematic inclination errors. Open points and dark-gray curves in the top panel represent the disk mass surface-density errors $\left(\Delta \ln \Sigma_{\mathrm{dyn}}\right.$, Appendix B.1) assuming the SVE is known to $10 \%$ - an optimistic scenario - and is flattened with $\alpha=0.7$. Line-types have the same meaning as for total mass; the dark-shaded area shows the range of $\Delta \ln \Sigma_{\text {dyn }}$ for $0.4<\alpha<1.0$ and the mid-point of the inclination error distribution. The light-gray shaded area represents systematic errors due to deprojection of $\sigma_{\mathrm{LOS}}$, discussed in $\S 3.5 .4$. The bottom panel repeats this calculation for $\Delta \ln \Sigma_{\mathrm{dyn}}$ assuming the SVE uncertainty is $50 \%$ (§2.1.1). Total mass errors are the same in the top and bottom panels.

The right panels of Figure 5 repeat the logarthmic errors in total-mass and disk surfacedensity, but adopt inclination and inclination errors from inverting the Tully-Fisher relation (Appendix A.2). Logarithmic errors in total mass (black horizontal lines) are given for two assumptions of the observed TF scatter ( 0.1 and $0.3 \mathrm{mag}$, labeled) and a TF-slope of -7 , suitable in red bands. Black, dotted, horizontal lines give the fractional error for $0.3 \mathrm{mag}$ scatter and TF slopes of -5 and -9 . This range of slopes and scatter cover the banddependent results in the literature. Best results are for the $K$-band TF found by Verheijen (2001) with a -9 slope and a scatter of order 0.2 mag. Logarithmic errors in disk mass surfacedensity are indicated by gray, shaded regions for $10 \%$ (top) and $50 \%$ (bottom) uncertainty 
in the SVE for a range of ellipsoid ratios $0.4<\alpha<1.0$, a TF slope of -7 , and 0.3 mag scatter. The solid, bisecting line assumes $\alpha=0.7$. Dashed curves show the full range of TF scatter and slopes for $\alpha=1$, while the dotted curve does the same for $\alpha=0.4$. For both $\Delta \ln \mathcal{M}_{\text {dyn }}^{\text {tot }}$ and $\Delta \ln \Sigma_{\text {dyn }}$, the precision is more sensitive to the scatter than the slope of the TF relation.

Disk-mass errors are rather flat for $i>15^{\circ}$ for $10 \%$ ellipsoid errors. This would argue for going to inclinations larger than $40^{\circ}$ to reduce total-mass errors. However, working against this arguement is the fact that systematic errors in $\Sigma_{\text {dyn }}$ continue to increase rapidly with inclination. Hence we conclude that if we can determine the SVE to $10 \%$, we should select galaxies with $30^{\circ}<i<40^{\circ}$ to equalize total- and disk-mass errors each at about $13 \%$. A more reasonable estimate is that our SVE errors will be closer to 20-30\% (Westfall 2009). This would indicate lower inclinations of $25-35^{\circ}$ to match total- and disk-mass errors at about $1.5 \times$ higher levels $(20 \%)$.

Note, however, that errors in Figure 5 are lower limits. Although additional photometric and kinematic errors are unlikely to dominate the total-mass error budget for inclinationcontributed errors above $\sim 10 \%$, we know already that there is a $25 \%$ error contribution to disk-mass errors from estimating the vertical scale-height $z_{0}$ alone (§2.2). Using iTF inclinations looks very powerful for reducing errors in both disk-mass and total mass to levels well below other contributed errors (10\%).

For iTF inclinations to be effective (i) very low inclination galaxies are needed to reduce disk-mass errors, and (ii) a clean identification of what kind of galaxies lie on a low-dispersion TF relation is needed to reduce total-mass errors. The latter implies the shape of the outer rotation-curve needs to be well-defined, which means $\mathrm{H}$ I velocity map is required, and that occurring warps are well understood (see discussion in Verheijen 2001). With better SVE estimates, a wider range of inclinations can be used below a given error threshold in diskmass. For $10 \%$ errors in both mass quantities, inclinations below $15^{\circ}$ and $25^{\circ}$ are needed for SVE errors of $50 \%$ and $10 \%$, respectively. Targets cannot be so face-on that their projected velocity gradients are unobservable. For $V_{\text {flat }}=250 \mathrm{~km} \mathrm{~s}^{-1}$, inclinations have to be above $11^{\circ}$ to keep the projected velocity a factor of 2 above the turbulent motions $\left(\sim 25 \mathrm{~km} \mathrm{~s}^{-1}\right)$. However, since we are not observing a line-width but a velocity field, the averaging of many spatial position allows for a velocity-centroid to be determined in the flat part of the rotation curve well below the actual dispersion due to, e.g., turbulent motions, as illustrated in Figure 16 of Paper I. Whatever the true inclination and circular speed, clearly the galaxy in that figure has a very regular velocity field and a flat asymptotic rotation curve from which an iTF-based inclination can be precisely derived. Despite this promise, in the absence of (ii), i.e., known accuracy, using iTF remains a compelling yet potentially fatal path if used in 
isolation.

To summarize, an optimal compromise for our survey goals can be reached by targeting galaxies with kinematically determined inclinations between $25^{\circ}$ and $35^{\circ}$. We do, however, take advantage of the iTF method to reduce errors in some situations (Andersen et al. 2010, in preparation).

\section{THE BROADENING FUNCTION}

Since $\sigma_{z}$ is at the heart of our measure of $\Sigma_{\text {dyn }}$, its estimation is arguably where we can have the greatest impact on minimizing errors. In Paper I we outlined two complementary approaches to deriving $\sigma_{z}$, with the intent of applying both in an iterative fashion to (i) optimize the determination of the SVE (using $\sigma_{\mathrm{LOS}}$ on individual fibers), and (ii) minimize template mismatch (using stacks of fibers in radial bins). We focus here on the latter because this method ultimately allows us to probe $\sigma_{z}$ at the largest radii. However, most of what is discussed is generic to both, or a coupled approach to determining $\sigma_{z}$.

Following the order of analysis, we proceed with determining the impact on the error budget given our method of fiber stacking (§3.1), deriving $\sigma_{\mathrm{LOS}}$ from cross-correlation methods (§3.2), and estimating random errors on $\sigma_{\mathrm{LOS}}$ due to spectral errors (§3.3) and template mismatch (§3.4). Corrections to $\sigma_{\mathrm{LOS}}$ that enable us to arrive at a reliable $\sigma_{z}$ estimate are described in (§3.5). These corrections compensate for effects of beam smearing, instrumental resolution, projection, and internal extinction. As a tertiary topic we consider the uncertainties in the spatial registration (including effects due to seeing variations) in $§ 3.6$. While positional registration of the IFU pointings is actually relevant to the initial fiber stacking, the uncertainties are negligible. Nevertheless, the discussion is included for completeness.

\subsection{Fiber Averaging: Ring "Stacking"}

Two-dimensional spectroscopic data can be binned spatially in a variety of ways to maximize S/N. Even for an analysis of the ellipsoid ratio, for example, we can divide the fibers into quadrants along major and minor axes, and average spectra in bins of radius. For the purpose of estimating $\Sigma_{\text {dyn }}$, with an estimate of the SVE shape already in hand, we take advantage of the near face-on geometry by combining fibers in azimuthal rings to measure a single $\sigma_{\mathrm{LOS}}$ at a given radius. The detailed assignment of fibers to a radial bin depends on a galaxy's inclination and position angle (PA), which we determine kinematically. Nominally the binning is done by radius, but we have also found it useful to bin instead by 
surface-brightness as this can be useful in isolating arm and inter-arm regions.

In any binning scheme, spectra are registered to take out the effects of projected rotation before co-addition. This is achieved by an iterative cross-correlation of each fiber spectrum against a suitably-broadened template. (The cross-correlation technique is described in detail in Paper III, and summarized here in the next section.) We work progressively starting with bins at the highest $\mathrm{S} / \mathrm{N}$, e.g., as a function of radius. On the first pass, we adopt a template between G9 III and K1 III, broadened to the dispersion of the previous bin. After determining the best template and broadening function for the stack (below), we adopt these as input for the registration, and iterate to convergence. The advantage of fiber averaging is that we are only required to determe a velocity centroid for each fiber in the average, instead of both centroid and width.

The spectral stacking algorithm has these specific steps: (1) Determine the best-fitting velocity offset for each fiber within a radial ring for a fixed velocity dispersion and provided template spectrum; (2) Shift all ring spectra to rest-frame velocity and combine them using weights defined by $(S / N)^{2}$; (3) Using the combined ring spectrum and the same template, determine the best-fitting velocity dispersion after fixing the velocity offset to be in the rest frame; and (4) Repeat steps 1-3, restarting with the updated velocity dispersion. Convergence is reached when the difference in subsequent determinations of the velocity offsets is below a specified threshold; here we set a threshold of $1 \mathrm{~km} \mathrm{~s}^{-1}$ except for rings with substantially low-S/N components. Noordermeer et al. (2008) have presented an alternate algorithm that alters the individual velocity offsets in order to minimize the velocity dispersion of the stacked spectrum. This is in contrast to relying on the best estimates of the velocity centroid for each fiber as we have done here. While the algorithms should be roughly equivalent at high $\mathrm{S} / \mathrm{N}$, we prefer our approach for low $\mathrm{S} / \mathrm{N}$ applications because velocity centroids are more precise. We discuss the impact of errors in the velocity registration on the derived broadening function in $\S 3.3$.

One of the ways to aid in the convergence and make the stacking process more robust at lower $\mathrm{S} / \mathrm{N}$ is to provide a prior estimate of the velocity offsets. We have compared priors including (a) the measured velocities of gas determined from the same spectra containing the stellar absorption (e.g., [O III] $\lambda 5007$ in the Mg Ib-region); (b) model velocities of the gas, based on a projected rotation-curve fit; (c) or the same, except for the stellar data in the inner region, extrapolated to larger radii. In all cases, we find that the resulting stacked spectra are identical over the full range of $\mathrm{S} / \mathrm{N}$ and radial bins. This implies that even though the stellar and gas velocities systematically differ (i.e., due to asymmetric drift), the iterative, cross-correlation process is robust to tune up the spectral registration. Indeed, without such iterations, the stellar spectra broaden systematically at radii where asymmetric-drift is large 
or the $\mathrm{S} / \mathrm{N}$ is low. This is significant because it means we may apply such priors to aid in the convergence of the velocity-registration of, e.g., stellar spectra in the Ca II-triplet region stellar spectra where emission lines are observed.

Figure 4 of Paper I shows examples of azimuthal rings for SparsePak and PPak observations of UGC 6918. The table inset gives the number of fibers in each radial bin as a function of radius, scaled to the radial scale-length. Figures 6 and 7 of this paper contrast the "before- and after-registration" spectra and cross-correlations for the $\mathrm{Mg}$ Ib region in one radial bin ( $r i n g$ ) of UGC 6918. This ring is between $2.6<\mathrm{R} / \mathrm{h}_{R}<3.4$, at a mean $V$-band surface-brightness of $21.6 \mathrm{mag} \operatorname{arcsec}^{-2}$. The data represent a cumulation of 2.25 hours of exposure, achieving a mean $\mathrm{S} / \mathrm{N}$ of 21 pixel $^{-1}$ in the coadded spectrum at roughly 0.5 mag below the Freeman disk central surface-brightness (Freeman 1970; UGC 6918 has a high surface-brightness disk).

As expected, the averaged spectrum and its associated cross-correlation have substantially lower noise than their individual elements. Particularly impressive is that after velocity registration not only is the correlation tightened (lines narrowed) but the noise is further reduced. This "noise" is due to the unregistered superposition of absorption lines. Indeed, the registration reveals many, weaker lines in the spectrum, and produces a cross-correlation that better matches that of the broadened template even outside of the correlation-peak where the broadening-function is determined. This comparison demonstrates the power of cross-correlation to pull out signal from the multi-fiber data distributed over a range of projected velocities. For comparison, if we were to limit ourselves to only those fibers that could be fit individually, the $\mathrm{S} / \mathrm{N}$ would drop by $\sim 20 \%$ because $40-50 \%$ of the fibers could not be fit at the limiting surface brightness of our data.

A final advantage of fiber averaging is the ability to mask out sky-lines. While these foregrounds are nominally subtracted from the data, there are often residuals due to imperfect match of the spectrograph aberrations between object and sky spectra, detector under-sampling, or simply the enhanced root-mean-square (RMS) due to the large number of counts in the sky line. All of these 'features' lower the $\mathrm{S} / \mathrm{N}$ in the specific wavelength region of the line; however the feature shifts in the galaxy rest-frame in a spatially-dependent manner because of the galaxy internal motions. Therefore by masking out the sky-line regions from the stack, it is possible to recover a continuous, line-free spectrum, optimized for $\mathrm{S} / \mathrm{N}$. This is particularly important in the Ca II-triplet region where sky lines are strong and prevalent. Figure 8 illustrates the masking method.

In this example, we have chosen only to mask out the strongest lines, typically those with peak flux more than twice the level of the sky continuum. Mask widths are $0.3 \mathrm{~nm}$ (roughly three times the instrumental full-width at half-maximum, FWHM), except for closely-spaced 
lines where the mask-width is reduced to leave inter-line gaps. Mask-widths are never less than 3 pixels. These mask widths were found empirically to maximize the resultant $\mathrm{S} / \mathrm{N}$ and, based on simulations, to minimize systematics in the derived broadening. (The skylines are unresolved, and the internal velocity shifts of the galaxy are always larger than the instrumental resolution.) The only region which does not appear to benefit from masking is in the molecular-band region from 860 to $870 \mathrm{~nm}$. Masking requires $\geq 105 \mathrm{~km} \mathrm{~s}^{-1}$ velocity spread in the Ca II-triplet region between fibers in a ring, achieved except in the inner-most ring for $80 \%$ of our $\mathrm{Mg}$ Ib sample and all but one galaxy observed in the Ca II-triplet region. The velocity spread is set simply by the largest mask-wdith.

\subsection{Deriving the Broadening Function $\sigma_{\mathrm{LOS}}$}

We apply a cross-correlation technique, rather than direct-fitting of the spectral data in wavelength space, to determine the broadening function and random errors on this broadening. While the two methods are equivalent in principle, in practice the information is projected in different ways (Simkin 1974). For example, in the direct-fitting approach, an assessment of template (mis)match is grossly evident in the detailed depth of various lines. In contrast, assessment of the broadening (mis)match is more readily obtained via inspection of the cross-correlation function since all of the signal for line profile shape is consolidated. Because the broadening is the primary signal of interest, we prefer the cross-correlation method, particularly because information on template mismatch (relevant for systematic errors) is still available in the cross-correlation using information outside of the peak (§3.4).

We have developed a new cross-correlation method, optimizing several technical attributes relevant to the accuracy and precision of the broadening measurement in our program. The analysis code is very general in the sense that it allows for Gauss-Hermite series decomposition of the cross-correlation peak (van der Marel \& Frank 1993) and input of any spectral template. For clarity, we focus in this paper on results for simple Gaussian broadening and templates based on measurements of single, Galactic stars. A complete discussion of the method is presented in Westfall (2009) and Paper III. We summarize here the salient features germane to the error-budget.

First, we construct a differential formulation based on convolution (rather than deconvolution; see Franx \& Illingworth 1988) to derive the broadening in a way that treats the templates and galaxy data in an identical, and symmetric fashion. To describe this we adopt the nomenclature in Paper III where o denotes cross-correlation, $\otimes$ denotes convolution, $G$ is the galaxy spectrum, $T$ is the template spectrum, and $B$ is the broadening function. We compare the cross-correlation of a broadened and redshifted template with an un-broadend, 
un-shifted template $\left(\mathrm{BTXC} \equiv X_{T} \equiv(T \otimes B) \circ T\right)$ to the cross-correlation of the galaxy spectrum with the un-broadened, un-shifted template $(\mathrm{XC} \equiv X \equiv G \circ T)$. We compare, in a $\chi^{2}$ sense, only the core of the correlation peaks, finding a region of 1.7 times the FWHM of the XC peak to be optimal in terms of precision. Our approach contrasts with earlier applications (see Rix \& White 1992 and Statler 1995 for reviews) which compare the XC with the un-broadend, un-shifted template auto-correlation $(\mathrm{AC} \equiv A \equiv T \circ T)$, or even deconvolve the XC peak directly. While mathematically equivalent, our approach starts with broadening the template, as is done in nature to the ideal template of the galaxy spectrum. Moreover, our approach should be immune to systematic effects due to "detector-censored" data, i.e., where the observed band-pass is finite. What this means in practice is that we do not have information from our templates about flux contributions due to broadening from outside of observed spectral window. We find this is particularly important when strong features of interest (e.g., Mg Ib or Ca II) are near the edge of the detected band-pass in either the template or galaxy spectrum. Tests indicate the impact of detector-censoring can lead to systematics in $\sigma_{\mathrm{LOS}}$ of order 10\% (Westfall 2009 and Paper III). By using convolution rather than deconvolution, we avoid filtering problems associated with Fourier transforms of noisy data.

Second, the fitting procedure allows for the masking of source emission-lines and skylines, handled symmetrically for template and galaxy spectra. Emission-line masking is critical in the $\mathrm{Mg} \mathrm{Ib}$ region not only for the $[\mathrm{O}$ III $] \lambda \lambda 4959,5007$ doublet but also for the weaker $[\mathrm{N} \mathrm{I}] \lambda \lambda 5198,5200$ doublet (Figure 6). Sky-line masking is critical in the Ca II-triplet region (Figure 8). Masking also enable us to isolate spectral regions of interest, e.g., the Mg Ib-triplet versus weaker Fe features in this region, or Ca II-triplet versus Paschen-series lines in the near-infrared, as we discuss below. The inability to mask spectral features with previous versions of cross-correlation software has often been touted as a primary advantage of directing-fitting methods (e.g., Rix \& White 1992). However, in our cross-correlation formulation masking is neither conceptually difficult or computationally challenging.

A relevant detail for Fourier-transform cross-correlation techniques and masking concerns tapering (apodization) of the mask edges to avoid high-frequency ripples. When the number of masks is large relative to the number of spectral channels, the tapering function appreciably diminishes the cross-correlation signal. Because of the identical and differential way we treat the template and galaxy correlation functions, we expect that a ripple should not have an impact on the derived broadening parameters. Simulations bear out the expectation that changing the tapering function does not alter the accuracy of the recovered parameters, but precision is improved by eliminating tapering, or at most by applying a 2-pixel cosine taper. (Each pixel is between 7 and $12 \mathrm{~km} \mathrm{~s}^{-1}$.) 
Third, the fitting is iterative in two significant ways: (1) in the optimization of the broadening, velocity-shifting of the template and the mask placement in the two (template and galaxy) reference frames; and (2) in fitting a low-order spectral continuum to the residuals between the broadened, shifted template and the galaxy spectrum in wavelength space, and then removing this residual from the observed galaxy spectrum (mathematically equivalent to adding the residual to the broadened template, but computationally simpler). The latter accounts for any low-frequency spectral mismatch between the template and galaxy due to, e.g., in order of likely significance: stellar mix, illumination correction, color-terms in flux calibration, reddening, or nebular continuum emission. This continuum correction is important because while the low order spectral shape does not contribute directly to the width or shape of the cross-correlation peak, it does impact the cross-correlation function at lower frequencies, which in turn alters the amplitude of the correlation peak. This mismatch has impact on the differential comparison of the BTXC and XC even for the high spectralfrequency component (e.g., consider the effect of baseline variations near the correlation-peak core), which fundamentally is the only quantity of interest for $\sigma_{\text {LOS }}$. The mismatch also alters the goodness-of-fit assessment of the template (§3.4).

Finally, the fitting process includes evaluation of the error spectra, and the computation of a full covariance matrix for determining errors on the fitted parameters. These error estimates have been tested against simulations, and found to be accurate and robust. Comparison of the simulations with real data is described below.

\subsection{Random and Systematic Errors on $V_{\mathrm{sys}}$ and $\sigma_{\mathrm{LOS}}$ due to Spectral Noise}

We focus here and in the next subsection on simulations matched to the SparsePak observations of two galaxies from our pilot program, taken in both $\mathrm{Mg}$ Ib and Ca II-triplet regions: UGC 11356, a well-studied giant elliptical galaxy; and UGC 6918, a high surface-

brightness spiral galaxy in our sample. The former was observed for the purpose of comparing our measurements to those in the literature. It should also contain a relatively simple stellar population yielding similar kinematics in the two spectral regions - albeit with $\sigma_{\mathrm{LOS}}$ substantially larger than for our survey sources. UGC 6918 has $\sigma_{\text {LOS }}$ values typical of our survey sources, but potentially illustrates a composite stellar system where systematic differences arise in the derived $\sigma_{\text {LOS }}$ between the two wavelength regions. These difference might occur due to variations in the dynamics of disk stellar populations correlating with age, and hence color.

We have carried out a set of Monte Carlo simulations to determine the accuracy and reliability of extracting the centroid velocity $\left(V_{\text {sys }}\right)$ and line-wdith $\left(\sigma_{\text {LOS }}\right)$ using our cross- 
correlation technique. These simulations use our stellar templates, observed at high $\mathrm{S} / \mathrm{N}$. These spectra are velocity shifted, broadened and noise-aberrated to span a range of $\mathrm{S} / \mathrm{N}$, $\sigma_{\mathrm{LOS}}$, and $V_{\text {sys }}$ encompassed by our survey data. We extended the range of simulated $\sigma_{\mathrm{LOS}}$ to higher values typical of the cores of giant elliptical galaxies. Independent, and more detailed simulations in the $\mathrm{Mg}$ Ib region applicable to UGC 6918 are given in Paper III.

As part of our analysis, we divide the Mg Ib-region into two spectral subregions, and considered these in addition to the full $\mathrm{Mg}$ Ib and Ca II-triplet spectral regions. These two subregions contain, respectively, the $\mathrm{Mg} \mathrm{Ib}$ triplet, and everything but the $\mathrm{Mg} \mathrm{Ib}$ triplet, as indicated in the K1 III spectrum in Figure 9. As this figure shows, the latter subregion is dominated by signal from many weak lines of Fe I, Ti I, Cr I, Fe II, Ti II, and TiO, in decreasing importance, prevalent in the cool stars expected to dominate the detailed linesignature in the integrated light of galaxies. This division was motivated by the results of Barth, Ho, \& Sargent (2002) indicating the Mg Ib-triplet was problematic for $\sigma_{\text {LOS }}$ measurements - plausibly due to abundance variations between stellar templates and integrated galaxy spectra. Inspection of Figure 9 shows that our Mg Ib subregion still contains narrow, weaker lines of Fe I and Fe II, as well as the TiO band-head. In particular, the bluer two lines of the $\mathrm{Mg}$ Ib triplet are significantly contaminated, as seen in the real galaxy spectra of Figure 6. The bluest line of the triplet coincides nearly with the TiO molecular band-head which is strong in stars cooler than M0 (see Figure 15 in Paper I). In terms of random errors on $\sigma_{\mathrm{LOS}}$, we find from our simulations that the two subregions have comparable $\mathrm{S} / \mathrm{N}$ in a cross-correlation sense, or about $1 / \sqrt{2}$ that of the full $\mathrm{Mg}$ Ib region. Otherwise both subregions yield similar systematic trends with $\mathrm{S} / \mathrm{N}$. For this reason, we do not distinguish these subregions further in discussion of $\mathrm{S} / \mathrm{N}$.

While we have studied simulations using a large range of stellar templates, we illustrate results using K1 III and M3 III templates here for clarity. These two stars, respectively, appear to be the best, or close to the best single-star templates in the Mg Ib and Ca IItriplet regions. This holds for all of the galaxies in our sample analyzed to date, as well as the elliptical UGC 11356, as we demonstrate below. The results of these simulations are shown in Figure 10a (for the K1 III template) and Figure 10b (for the M3 III template). Measurements from galaxy observations are also shown for comparison. These include all individual fibers in the Mg Ib region in UGC 6918 for which cross-correlation yielded successful measurements; the inner 14 fibers for UGC 11356 in both Mg Ib and Ca II-triplet regions; the same set of fibers in UGC 6918 for the Ca II-triplet region; and the 5 rings defined in Figure 4 of Paper I for UGC 6918 in both spectral regions.

The accuracy of the derived velocity centroid and broadening based on the simulations is superb, and well below the random errors for $\mathrm{S} / \mathrm{N}>3 \mathrm{pix}^{-1}$ (refer to panels in first and 
third columns of Figures 10a and 10b). This result is independent of broadening and centroid velocity. Below this $\mathrm{S} / \mathrm{N}$ level there is a hint that systematics begin to become significant, with positive velocity and velocity-dispersion offsets for smaller line-widths, and negative offsets at larger line-widths. This is clearly demonstrated by the more detailed simulations presented in Paper III. We conclude that at $\mathrm{S} / \mathrm{N}>3 \mathrm{pix}^{-1}$ systematic errors in the derived velocities and widths are negligible. We defer discussion of the accuracy of $\sigma_{\text {LOS }}$ derived from the galaxy observations until after consideration of the effects of template mismatch.

In terms of precision, to first order we find that $V_{\text {sys }}$ and $\sigma_{\mathrm{LOS}}$ errors scale inversely with $\mathrm{S} / \mathrm{N}$ and $\sigma_{\mathrm{LOS}}$ as expected (wider profiles yield less precise measures at a given $\mathrm{S} / \mathrm{N}$; refer to panels in second and fourth columns of Figures 10a and 10b). At very large $\sigma_{\mathrm{LOS}}$ there is some indication that the dependence on $\mathrm{S} / \mathrm{N}$ is somewhat stronger, at least for $V_{\text {sys }}$. There is very little dependence of these results on the simulation template. However, the simulations were fit with the correct template, so the effects of template-mismatch are absent in these results.

In contrast, the templates used to derive centroid velocities and broadenings for the galaxy observations may be mismatched. Indeed, comparison of measurements of simulations to those of real galaxy spectra shows the latter have errors twice as large in the $\mathrm{Mg}$ Ib region, yet similar errors in the Ca II-triplet region. Interestingly, at a given $\mathrm{S} / \mathrm{N}$ the errors derived from the simulations are 2 to 2.5 times larger in the Ca II-triplet region than in the $\mathrm{Mg} \mathrm{Ib}$ region. Assuming no template mismatch, we conclude that the $\mathrm{Mg} \mathrm{Ib}$ region in principle yields more precise kinematic measurements than the Ca II-triplet region at a given spectral continuum $\mathrm{S} / \mathrm{N}$. However, in practice the two regions yield comparable precision. It is plausible that the additional contribution of random error to the kinematic measurements in the $\mathrm{Mg}$ Ib region is due to template mismatch. This conjecture has some basis in the fact that errors in $V_{\text {sys }}$ and $\sigma_{\mathrm{LOS}}$ in the galaxy spectral data in the $\mathrm{Mg}$ Ib region increase substantially when they are fit with a template (M3 III) that is clearly not a good representation of the spectrum in that region. In contrast, the errors derived in the Ca II-triplet region appear relatively immune to the template applied.

On the basis of these simulations we conclude that the typical random error in our survey for $\Delta \ln \sigma_{\mathrm{LOS}}$ is $3 \%$, given a typical spectral continuum $\mathrm{S} / \mathrm{N}$ in the $\mathrm{Mg} \mathrm{Ib}$ region of 40 for an azimuthally-averaged spectrum (see Paper I and Figure 4 therein). Since the measurement of the broadening function is not yet corrected for other effects $(\S 3.5)$, for the purpose of book-keeping we refer to this quantity as $\sigma_{\text {obs }}$, and hence $\Delta \ln \sigma_{\text {obs }}=0.03$.

One final consideration concerns the impact of velocity centroid erros on the registration precision of the azimuthal averaging. In general centroid errors will systematically broaden the stacked spectrum. Most of our data has spectral continuum $\mathrm{S} / \mathrm{N}>2$ in the individual 
fiber spectra. Since the errors on $V_{\text {sys }}$ remain well below $\sigma_{\text {LOS }}$ (i.e., $\epsilon\left(V_{\text {sys }}\right) / \sigma_{\text {LOS }}<0.3$, Figures 10a and 10b) in this $\mathrm{S} / \mathrm{N}$ regime, azimuthal averaging introduces less than a few percent increase to the derived broadening. This is negligible for our purposes in this paper. However we do note that in our outer-most radial rings, where the $\mathrm{S} / \mathrm{N}$ for individual fibers is 1 to 2, our simulations indicate there could be as much as a $20 \%$ increase in the measured $\sigma_{\text {LOS }}$ due to registration errors.

\subsection{Errors on $\sigma_{\mathrm{LOS}}$ due to Template Mismatch}

Perusal of the literature reveals that single stars typically have been used as templates for cross-correlation analysis to study the dynamics of disk and spheroidal stellar systems. One critical question for our analysis is whether $\sigma_{\text {LOS }}$ is sensitive to the specific choice of template. Substantial discussion of the issue of template-mismatch can be found in Rix \& White (1992), Statler (1995), and references therein. Late-G or early-K giants are usually adopted, with the (often unstated) assumption that these stars dominate the kinematic signal in the integrated light of early and intermediate-type galaxies. This is certainly reasonable given the luminosity of red-giant and horizontal branch stars, and their apparent dominance of the integrated light of old stellar populations. For later-type disks (especially near their outskirts, or in the cores of vigorously star-forming systems), the relative youth of their stellar populations may alter the picture, both due to the prevalence of luminous, hot, young stars on, or near the tip of the Main Sequence and cool, intermediate-age giants (e.g., the AGB). The concern regarding hot stars may be tempered by virtue of their decreasing line-strengths from metals. Nonetheless, the question remains whether there are substantial systematics in $\sigma_{\mathrm{LOS}}$ from template-mismatch. We define the template-mismatch error to be $\Delta \ln \sigma_{\mathrm{tpl}} \equiv \Delta \sigma_{\mathrm{tpl}} / \sigma_{\mathrm{LOS}}$, where $\Delta \sigma_{\mathrm{tpl}}$ is the half-width of the full range of $\sigma_{\mathrm{LOS}}$ for all viable templates.

To motivate the importance of answering this question we illustrate in Figure 11 the measured $\sigma_{\mathrm{LOS}}$ for fibers in the core of UGC 11356 and UGC 6918 using a range of template stars from F0 to M5, all luminosity-class II-III (giants). The specific stars and their spectra are illustrated in Figure 15 of Paper I. Values are means over the individual fiber measurements, with errors given as the standard deviation of these measurements. The errors are within a factor of two from the mean estimated errors from the cross-correlation analysis, indicating little intrinsic variance between the regions sampled by the individual fibers. The range of template spectral types was chosen on the basis of direct visual inspection of the template and galaxy spectra. In the $\mathrm{Mg}$ Ib region, types earlier than $\mathrm{F} 0$ have insufficient line-strengths in both $\mathrm{Mg} \mathrm{Ib}$ and Ca II-triplet regions. While types later than M0 have a 
strong molecular band-head in the $\mathrm{Mg}$ Ib region which is not observed, types as late as M5 are acceptable in the Ca II-triplet region. We extended our template range accordingly. We have limited templates here to luminosity class II-III stars, based on astrophysical prejudice for what stellar types with strong lines are most likely to dominate the integrated light of galaxies. Furthermore, we choose mostly solar metallicity stars given the reasonable assumption that the integrated light of disks is dominated by Population I stars. Nevertheless, we include one sub-solar metallicity star (HR 4695), at intermediate spectral type, to probe the validity of the latter assumption.

The left-hand column of Figure 11 demonstrates that template mismatch is very significant in spirals and ellipticals, and in both spectral regions. While trends of $\sigma_{\text {LOS }}$ with spectral type are different for the two spectral regions, they are qualitatively similar for both galaxies. Compared to the full $\mathrm{Mg}$ Ib region, we find the systematic trends in $\sigma_{\text {LOS }}$ with template are twice as large in the subregion isolating the Mg Ib-triplet, while the sub-region excluding the $\mathrm{Mg}$ Ib triplet has a smaller range. The two Mg Ib sub-regions also have different qualitative trends. These differences are largely due to the appearance of the TiO band-head in cool stars later than mid-K, located near the bluest of the Mg Ib triplet lines. Given the increased random errors (§3.3) in $\sigma_{\mathrm{LOS}}$ by limiting the spectral range to either of these subregions, we do not considered them further here. However, we note the added information by definning such subregions can be exploited to further optimize template-matching in the highest S/N regimes.

One method for limiting the impact of template mismatch on $\sigma_{\mathrm{LOS}}$ is to restrict the template spectral range with a notional argument, e.g., based on colors or stellar population synthesis (SPS) models. For example, were we to restrict the templates to F8-M2 or G8-K4 ranges, we would obtain $\Delta \ln \sigma_{\mathrm{tpl}}=0.12$ and 0.07 respectively. Indeed, more recent studies (e.g., Falcón-Barroso et al. 2006) use SPS models to directly fit the spectral continuum (effectively color) and line-strength. In principle this option is open to us, but until recently the stellar libraries have had insufficient spectral resolution for our purposes. The one exception is PEGASE-HR (Le Borgne et al. 2004), based on the ELODIE library observed at a resolution of $R=42,000$. Unfortunately, the models degrade the resolution to $R=10,000$ for a Gaussian instrumental profile; this is too low for many of our $\mathrm{Mg}$ Ib observations, which often have non-Gaussian instrumental profiles. Further, the library does not extend far enough to the red to reach the Ca II-triplet. Nonetheless, improved models like these are highly desirable in the future.

Even with suitable high-resolution SPS models, properly modified for our instrumental broadening, there remains the issue of degeneracy - in a photometric sense - between equally suitable models with a wide range of model parameters (e.g., age, metallicity, star-formation 
history). The problem here is that it has not yet been demonstrated that this photometric degeneracy has an equivalent kinematic degeneracy. Specifically, the amplitude of $\Delta \ln \sigma_{\text {tpl }}$ has not been quantified using the direct-fitting SPS approach in any study presented in the literature.

For the above reasons we proceed here with a simple analysis based on a set of single template stars. These are observed with the same instrument and same instrumental configuration (often observed on the same night) as used for our target galaxies. We define a set of indices that allow us to minimize the impact of template-mismatch on $\sigma_{\text {LOS }}$, quantify $\Delta \ln \sigma_{\mathrm{tpl}}$ in this context, and conclude with a brief discussion of how this approach can be further improved.

\subsubsection{Template mismatch indices}

The function $\chi_{\nu}^{2}(X C)$ which is minimized to determine the optimum broadening, $\sigma_{\mathrm{LOS}}$, is the error-normalized RMS between the XC and BTXC, taken in the usual $\chi^{2}$ sense, but measured only within a small fitting window of the cross-correlation (1.7 times the FWHM of the cross-correlation peak). We find that $\chi_{\nu}^{2}(X C)$ is highly insensitive to changes in the template, even though the derived $\sigma_{\text {LOS }}$ varies substantially. This appears a worrisome fact for the cross-correlation approach, but since the Fourier transform does not throw out intrinsic information, sensitivity to template mismatch must be present somewhere in the cross-correlation function outside the fitting window. Inspecting simulations, we concluded that (i) the relative heights of the $\mathrm{XC}$ and $\mathrm{BTXC}$ give information on the match of the average line-depth (equivalent width) in the template versus galaxy spectra; while (ii) the "RMS" amplitude and asymmetry of the cross-correlation outside the fitting window give information on the match of the relative line-depths between the two. Based on this, we developed two indicators based on the cross-correlation function, and a third based on the direct spectrum to compare direct-fitting vs cross-correlation approaches. All three of these indices are illustrated in Figure 11 for the optimum broadening for each template:

1. XC-rms is the RMS between the $\mathrm{XC}$ and BTXC. It is like $\chi_{\nu}^{2}(X C)$, except it's not error-normalized, i.e., it is not $\chi^{2}$, and is computed over the full correlation range. This range is nominally the same for all templates unless the fitted velocities are substantially different. However, XC-rms is normalized by the amplitude of the crosscorrelation peak to take into account the trend of stronger correlation peaks (and hence asymmetry with later spectral types).

2. $\mathrm{A}_{\mathrm{N}, \mathrm{c}}$ is the RMS asymmetry $(\mathrm{A})$ of the $\mathrm{XC}$ when mirrored about its fitted velocity 
centroid. It is an index of the "lopsidedness," or lack of mirror-symmetry of the cross-correlation, both at small and large lags. Like XC-rms, it is also normalized (N) by the amplitude of the cross-correlation peak. It is further corrected (c) for the asymmetry of the similarly-normalized cross-correlation of the broadened template with the un-broadened template (BTXC). This accounts for the non-intuitive (but mathematically correct) result that in the presence of detector censoring (i.e., any finite spectral window) the cross correlation of a template with its broadened counter-part has some intrinsic non-zero amount of asymmetry. The correction is small $(<10 \%)$. With the exception of the correction, $A_{N, c}$ is equivalent to the inverse of the term " $R$ " defined by Nelson \& Whittle (1995).

3. $\chi_{\nu}^{2}(\lambda)$ is the error-weighted RMS between the observed galaxy spectrum and the bestfitting broadened template, based on the cross-correlation and continuum fitting techniques, as described above. This index is independent of the fitting process that determines the velocity broadening, but is otherwise equivalent to what is used in direct-fitting methods.

Both XC-rms and $\mathrm{A}_{\mathrm{N}, \mathrm{c}}$ have larger dynamic range given their relative scatter than $\chi_{\nu}^{2}(\lambda)$. The insensitivity of $\chi_{\nu}^{2}(\lambda)$ to variations in template raises the possibility that templatemismatch errors in direct-fitting methods may be substantial. The indices XC-rms and $\mathrm{A}_{\mathrm{N}, \mathrm{c}}$ exhibit similar template resolution for both galaxies and for a given spectral region. Consequently, application of these indices (described below) to a large number of galaxy spectra yield quantitatively comparable results for $\Delta \ln \sigma_{\mathrm{tpl}}$. Because of the greater simplicity and intuitive nature of the XC-rms definition we adopt it in preference to $\mathrm{A}_{\mathrm{N}, \mathrm{c}}$.

\subsubsection{Index application and performance}

In practice, the above indices can be used to minimize $\Delta \ln \sigma_{\text {tpl }}$ by identifying the template with the minimum index value, defining a confidence interval based on the errors in that index, and then averaging $\sigma_{\mathrm{LOS}}$ for all templates with index values within this confidence interval. Variance in $\sigma_{\text {LOS }}$ can also be determined for this same template subset, and $\Delta \ln \sigma_{\text {tpl }}$ quantified in a well-defined manner. For the data in Figure 11, error bars on these indices are based on the measured variance between groups of fibers, and are likely over-estimates, for reasons given below. Nonetheless they are suitable for demonstrating the outlined technique to minimize the impact of template-mismatch. We adopt a " $1 \sigma$ " confidence interval in the sense that a template index must be no greater than the quadrature sum of the minimum index value and the $1 \sigma$-errors on both the minimum and template index values. 
As anticipated in the preceding discussion, $\mathrm{XC}-\mathrm{rms}$ and $\mathrm{A}_{\mathrm{N}, \mathrm{c}}$ are substantially superior to $\chi_{\nu}^{2}(\lambda)$ in terms of $\Delta \ln \sigma_{\text {tpl }}$. A feature which appears to be problematic for $\chi_{\nu}^{2}(\lambda)$ is the selection of templates at disparate temperatures in each spectral region. This not only increases $\Delta \ln \sigma_{\mathrm{tpl}}$, but without some deeper understanding of what causes these selection discontinuities in spectral type using $\chi_{\nu}^{2}(\lambda)$, it is hard to understand how to move forward to improve the situation; one is tempted to abandon $\chi_{\nu}^{2}(\lambda)$ and its associated direct-fitting approach as we have done here. One clue for future efforts may be that the low-metallicity star HR 4695 shows unusually low $\chi_{\nu}^{2}(\lambda)$. It is highly unlikely that massive and luminous galaxies have integrated light with sub-solar metallicity. Indeed, many galaxies in our sample have high values of $\mathrm{XC}-\mathrm{rms}$ and $\mathrm{A}_{\mathrm{N}, \mathrm{c}}$ for this same star, i.e., it provides a worse-fitting template, consistent with astrophysical expectations. Template-mismatch sensitivity to metallicity is definitely a desirable feature of any analysis attempting to understand the dynamics and stellar populations of galaxies.

Because we have a large, comprehensive template library, we can safely assume there exists a linear combination of templates that, once broadened, accurately represent any observed galaxy absorption-line spectrum. Here, we will make the further, simplifying, assumption that at least one of the templates alone is a suitable match to the observed galaxy spectrum. By this we mean that there exists one template which produces minimal systematic error in the derived broadening due to template-mismatch. In future papers in this series we substantiate that these are both good assumptions. In using an index-minimization approach, where the indices themselves are subject to random errors, the impact of templatemismatch on $\sigma_{\text {LOS }}$ uncertainties - what we have defined as $\Delta \ln \sigma_{\mathrm{tpl}}$ - is a random process. In other words, we would get a different value for $\sigma_{\mathrm{LOS}}$ for the same set of templates for repeat measurement of a galaxy spectrum. Hence for our application $\Delta \ln \sigma_{\text {tpl }}$ is a random error.

Using the individual fiber measurements for UGC 11356 and UGC 6918, we illustrate in Figure 12 the relative amplitudes of random errors due to shot noise in the spectra $\left(\Delta \ln \sigma_{\mathrm{LOS}}\right)$ and random errors due to template mismatch $\left(\Delta \ln \sigma_{\mathrm{tpl}}\right)$. As we found earlier, $\Delta \ln \sigma_{\mathrm{LOS}}$ is inversely proportional to $\mathrm{S} / \mathrm{N}$. By applying the XC-rms index, we dramatically lower the errors associated with template mismatch, essentially eliminating it for Ca II-triplet region measurements, and making these errors comparable to $\Delta \ln \sigma_{\mathrm{LOS}}$ in the $\mathrm{Mg} \mathrm{Ib}$ region. The correlation between the two sets of errors is related to the fact that errors on $\sigma_{\mathrm{LOS}}$ and $\mathrm{XC}-\mathrm{rms}$ for a single spectrum and template correlate in the same way with $\mathrm{S} / \mathrm{N}$.

As verification of our template-mismatch minimization method, we show in the right panel of Figure 12 the difference between individual fiber measurements made in the $\mathrm{Mg} \mathrm{Ib}$ and Ca II-triplet regions at similar physical locations for our two example galaxies. The 
velocity dispersion measurements are statistically identical as we would expect for the elliptical and also the spiral - if age-dynamical variations in disks are small. Figure 11 shows that this result need not necessarily be the case without some proper identification of suitable template. By extension, we may postulate this result as an initial confirmation that age-dynamical variations for spirals are in fact small, as observed in their integrated opticalnear-infrared light. It is also worth noting that our measurement of the absolute value of the velocity dispersion in UGC 11356 agrees within the errors with previous results in both the Mg Ib region (Bender et al. 1994, Fisher 19997, Gerhard et al. 1998) and Ca II-triplet region (Nelson \& Whittle 1995). This is significant since our best-fitting template in the $\mathrm{Mg} \mathrm{Ib}$ region is similar to what was used in previous studies. In the Ca II-triplet region our best-fitting template is considerably latter than those observed by Nelson \& Whittle (1995), but we find little change in the derived broadening when going to these earlier stellar types.

To apply our method in general we need to calibrate XC-rms errors as a function of $\mathrm{S} / \mathrm{N}$. Based on simulations, we illustrate in Figure 13 how the logarithmic errors on XC-rms depend on S/N. Errors for the other indices were computed in the same way. These errors are only due to random errors added to the simulated spectra, i.e., there is no template mismatch between the simulated BTXC and XC. We find logarithmic errors are only weakly dependent on line-width and $\mathrm{S} / \mathrm{N}$, which is convenient for the application we have described.

To determine if these simulations are realistic we took groups of fibers at similar radii and S/N (hence surface-brightness) in UGC 11356 and UGC 6918 separately, as illustrated in Figure 11. We computed the variance in the indices, and plotted them accordingly in Figure 13. We interpret these as upper limits to the index errors since real variations between the spectra may exist, e.g., stellar population non-uniformity. These "measurements" of $\Delta \ln$ $\mathrm{XC}-\mathrm{rms}$ are roughly twice as large as the values estimated from simulations. We adopt $\Delta \mathrm{ln}$ $\mathrm{XC}-\mathrm{rms}=0.06 \pm 0.02$ and $0.12 \pm 0.04$ based on simulations and measurements, respectively, over a $\mathrm{S} / \mathrm{N}$ range typical of observed, azimuthally-averaged spectra.

Results of applying this calibration of XC-rms errors to azimuthally-averaged spectra of 7 representative galaxies 2 from our survey are shown in Figure 14. The ring spectra have $\mathrm{S} / \mathrm{N}$ from 7 to 200 per pixel. The median random error on $\sigma_{\mathrm{LOS}}$ due to shot noise is $3 \%$, with $75 \%$ of the sample having errors $<5 \%$; random errors follow the trend and zero-point with $\mathrm{S} / \mathrm{N}$ as seen for the individual fibers. The median value is a restatement of our result in the previous section for $\Delta \ln \sigma_{\mathrm{LOS}}$. The median random errors due to template mismatch are $4 \%$, with $75 \%$ of the sample having errors $<5 \%$ assuming $\Delta \ln$ XC-rms is what is observed, i.e., about 0.12 , or a factor of two larger than the simulation value. We adopt the median

\footnotetext{
${ }^{2}$ UGC 463, 4555, 5180, 6128, 6869, 6918, and 10443.
} 
value, i.e., $\Delta \ln \sigma_{\mathrm{tpl}}=0.04$.

Were we to adopt a single template for all galaxies and all radial bins, e.g., a K1 III star for the $\mathrm{Mg}$ Ib region, our derived $\sigma_{\mathrm{LOS}}$ would be low, on average, by about $2 \%$, with a standard deviation of $8 \%$. There is a hint of a trend with radius such that a K1 III star is either too early or too late at the inner-most and outermost radii, respectively. Adopting a K1 III star is not a bad choice, but with the indices described here, we are able to largely eliminate systematic error due to template mismatch.

\subsubsection{Future improvements}

Our analysis can be improved in the following ways: (a) by including a wider range of metallicity for stars already in our existing spectral library; (b) by allowing for multiple templates in each radial bin, either single-star templates or multi-component SPS model templates based on our library; (c) by enabling each of these spectral components to have their own separate kinematic broadening (e.g., de Bruyne et al. 2004); and lastly (d) by treating the $\mathrm{Ca}$ II-triplet and $\mathrm{Mg}$ Ib regions simultaneously, in a self-consistent way such that the stellar population synthesis is consistent with the observed, de-reddened colors and spectra, while yielding an identical velocity dispersion for a given template component in both spectral regions (see $\S 4.3 .2$ and Figure 7 of Paper-1). These are refinements for future work.

\subsection{Corrections to $\sigma_{\mathrm{LOS}}$}

\subsubsection{Instrumental resolution}

The instrumental dispersion, $\sigma_{\text {inst, }}$ is due to a combination of the finite fiber aperture and spectrograph optical aberrations 3 We measure $\sigma_{\text {inst }}$ via Gaussian line-fitting to the line-lamp spectra. For any given observational configuration we typically identify 50 or more lines over the full spectral range which have adequate $\mathrm{S} / \mathrm{N}$ and are un-blended. The widths of these lines are determined to high precision (a few \%), and provide an exquisite map of the instrumental broadening as a function of spatial and spectral position (the former is equivalent to fiber \#). We find a mean broadening due to instrumental resolution of $\sigma_{\text {inst }}=10.8 \pm 1.5 \pm 2.2$ and $13.2 \pm 1.9 \pm 2.4 \mathrm{~km} \mathrm{~s}^{-1}$ in the $\mathrm{Mg} \mathrm{Ib}$ and $\mathrm{Ca}$ II-triplet regions

\footnotetext{
${ }^{3}$ The instrumental resolution defined as the FWHM, or $2.35 \sigma_{\text {inst }}$.
} 
respectively for SparsePak, and $\sigma_{\text {inst }}=18.8 \pm 1.7 \pm 1.4 \mathrm{~km} \mathrm{~s}^{-1}$ in the Mg Ib region for PPak, where the two sets of "errors" for each value are the characteristic range of broadening in the spatial and spectral dimensions. Subtle changes in spectrograph camera focus on different observing runs produced different patterns of instrumental resolution with wavelength and spatial position; these variations are within the quoted range.

Instrumental-broadening corrections for the ionized-gas line-widths are determined directly based on the widths from line-lamp spectra closest to the observed wavelength of the ionized-gas line in the galaxy spectrum. Since our measurement of line-width from the stellar spectroscopic data are achieved via cross-correlation against stellar templates observed with the same or similar instrumental configuration, to first order the instrumental broadening is taken into account. This accounting is imperfect due to redshift, the fact that stellar templates were not observed in every fiber, and because of focus variations between observing runs.

The first two of these effects produce very modest differences between the instrumental broadening of template and galaxy spectra: Galaxy redshifts are low $(z<0.042$; corresponding to shifts typically $25 \%$ of the observed wavelength range), the templates were observed with many fibers spanning the slit, and the instrumental broadening varies little over the spectral and spatial range of the data. Consequently, the mismatch in $\sigma_{\text {inst }}$ is $<1 \mathrm{~km} \mathrm{~s}^{-1}$ and varies from fiber to fiber. However, run-to-run changes in spectrograph focus have led to systematic differences between the template and galaxy instrumental resolutions, $\sigma_{\mathrm{T}}^{\text {inst }}$ and $\sigma_{\mathrm{G}}^{\text {inst }}$ respectively. We define $\delta \sigma_{\text {inst }}^{2} \equiv\left(\sigma_{\mathrm{G}}^{\text {inst }}\right)^{2}-\left(\sigma_{\mathrm{T}}^{\text {inst }}\right)^{2}$, and apply a correction of the form $\sigma_{\mathrm{LOS}}^{2}=\sigma_{\mathrm{obs}}^{2}-\delta \sigma_{\text {inst }}^{2}$. Note that $\delta \sigma_{\text {inst }}^{2}$ can be positive or negative.

For SparsePak, we estimate $\delta \sigma_{\text {inst }}^{2} \sim 0.0$, and so we do not apply a correction for instrumental mismatch. The remaining mismatch due to redshift and fiber sampling effects are less than a quarter of the range of observed instrumental broadening with wavelength and fiber, or $<0.5 \mathrm{~km} \mathrm{~s}^{-1}$, from which we arrive at $\Delta \ln \sigma_{\text {inst }}=0.04$ for SparsePak.

For PPak, $\delta \sigma_{\text {inst }}^{2}$ is often not zero. A typical value of $\sqrt{\left|\delta \sigma_{\text {inst }}^{2}\right|}$ is $6 \mathrm{~km} \mathrm{~s}^{-1}$. With the effort of measuring line-lamp spectra line-widths, we are able to determine this quantity to better than $0.4 \mathrm{~km} \mathrm{~s}^{-1}$. The uncertainty includes the remaining mismatch due to redshift and fiber sampling effects. Hence $\Delta \ln \sigma_{\text {inst }}=0.02$ for PPak.

What is relevant for the error budget of $\Sigma_{\text {dyn }}$ is the quantity $\left(\sigma_{\text {inst }} / \sigma_{\text {obs }}\right)^{2} \Delta \ln \sigma_{\text {inst }}$ (see $\S 3.5 .6$ and Appendix B). In this context, the uncertainties introduced by $\sigma_{\text {inst }}$ are the same for both instruments. We adopt an equivalent, median value of $\sigma_{\text {inst }}=15 \mathrm{~km} \mathrm{~s}^{-1}$ and $\Delta \ln \sigma_{\text {inst }}=0.03$ for calculations below. Overall, the effects are systematic for a single fiber for a given galaxy, but random when averaged over a stack of fibers. Similarly, the effects 
are random when averaging over observing runs for a single fiber and galaxy.

\subsubsection{Beam smearing}

Beam-smearing arises from the projected intensity, velocity and velocity-dispersion gradients across the SparsePak and PPak fiber faces, or "beams," suitably broadened for seeing. Features of fiber-optical spectroscopic data that differ from aperture-synthesis measurements at radio wavelengths and from direct-imaging spectroscopy include the discrete nature of the beams and the azimuthal scrambling properties of fibers. Compared to imaging-spectroscopy, the scrambling property of fibers ensures that the observed line-width is independent of the direction of the velocity gradient.

Begeman (1989) describes the general convolution process that relates an observed moment of the velocity distribution function (VDF) to the intrinsic distribution function, assuming the VDF is Gaussian at any given spatial position. This study arrives at a Taylor-series approximation to the convolution integral relating the observed and intrinsic velocity fields. While this can be generalized for non-Gaussian VDFs and higher-order moments, the validity of a Taylor-series approximation must be evaluated in each specific application. We follow the general convolution scheme of Begeman (1989) but depart from this formalism in several ways.

First, we employ an iterative scheme which starts with a smooth (polynomial model) characterization of the observed velocity and velocity dispersion fields. (In future analysis we extend this scheme to include higher-order moments, e.g., in the context of a Gauss-Hermite expansion of the VDF.) We adopt this characterization as the initial estimate of the intrinsic distribution, and as such the scheme is well-posed. We then synthetically "observe" it with the appropriate fiber foot-prints convolved with an estimate for the seeing and instrumental resolution. Initial correction factors are then estimated to be the ratio of the synthetic observations to the estimated intrinsic distributions. The data is corrected, fiber by fiber or ring by ring, based on this initial beam-smearing estimate, re-characterized, and then re-observed. The process is repeated until the corrections converge. The correction scheme requires no approximation.

Second, while our beam-smearing corrections are multiplicative, and whereas Begeman (1989) defined a linear (additive) correction, for our error analysis here we define an equivalent quadrature beam-smearing correction, $\sigma_{\text {beam }}^{2}$. This quantity is equal to the quadrature difference between the observed velocity dispersion $\left(\sigma_{\text {obs }}\right.$ corrected for instrumental broadening) and the corrected velocity dispersions $\left(\sigma_{\mathrm{LOS}}\right)$. We make this definition to parallel 
the corrections for instrumental resolution and to provide intuition in terms of a Gaussian convolution approach (Westfall 2009).

A description of the trends in $\sigma_{\text {beam }}^{2}$ with radius and azimuth serve to illustrate the amplitude of, and variation in, this correction. As a percentage effect, beam-smearing increases rapidly with radius and then declines after the roll-over from the steep inner rise of the rotation curve; the peak and roll-over occur typically within one disk scale-length. The corrections are typically under $4 \%$ for PPak data and under $7 \%$ for SparsePak data. In an absolute sense, we find that beam-smearing is strongly dependent on the amplitude and shape of the rotation curve in the centers of our pilot-survey sample galaxies. With SparsePak, beam-smearing produced by velocity gradients alone are $<5 \mathrm{~km} \mathrm{~s}^{-1}$ in the center and drops to $\sim 2 \mathrm{~km} \mathrm{~s}^{-1}$ on the minor axis at several scale-lengths, and below $<1 \mathrm{~km} \mathrm{~s}^{-1}$ on the major axis. While these numbers are representative of the survey as a whole, this is a conservative upper limit because the majority of observations were taken with PPak which has smaller fibers, and because it does not account for velocity dispersion gradients.

In general, beam-smearing corrections are determined by a complicated interplay between velocity and velocity dispersion gradients across the fiber faces. For example, at small radii where the rotation velocity is small but the velocity dispersion is large, beam-smearing corrections $\sigma_{\text {beam }}^{2}$ can even be negative. As another example, the crowding of isovels tends to make beam-smearing proportionally largest along the kinematic minor axis. However, the changing projection of the SVE dampens this effect for small values of $\alpha$ because the instrinsic azimuthal variation in $\sigma_{\mathrm{LOS}}$ is maximized.

Uncertainties in beam-smearing corrections arise from three sources of uncertainty: the shape of the SVE, seeing, and the flux distribution. The SVE shape, however, modulates the azimuthal dependence of the beam-smearing amplitude, not the mean value at a given radius. Consequently there is no impact of the a priori unknown SVE shape on the mean radial trend of $\sigma_{\mathrm{LOS}}$, estimated either from stacked rings or individual fibers. In contrast, when measuring the SVE shape itself, the azimuthal modulation in beam-smearing is crucial to determine; we will explore this in later papers in this series. The seeing dependence of $\sigma_{\text {beam }}^{2}$ is quite modest for SparsePak because of its large fibers and the good delivered image quality of the WIYN Telescope. For PPak observations the seeing values are well known.

Finally, uncertainties in the beam-smearing corrections also arise if the validity of our assumptions of the flux distribution across a fiber "beam" are suspect. While the stellar distribution in space and velocity is certainly smoother than that of the gas, the fibers subtend large physical scales. Considering the distances to our targets, the distributions of physical beam sizes have median, upper and lower-quartile values of $1.45_{-0.5}^{+0.65} \mathrm{kpc}$ for SparsePak and $0.83 \pm 0.3 \mathrm{kpc}$ for PPak, respectively. Beam sizes are under 2.9 and $1.7 \mathrm{kpc}$, 
respectively, for SparsePak and PPak for $90 \%$ of the sample. For the galaxies in the sample, there are typically between 2-3 and 4-5 fibers per radial disk scale-length for SparsePak and PPak respectively. Nonetheless, we find no significant difference between $\sigma_{\text {beam }}^{2}$ calculated assuming a uniform flux distribution and calculated assuming an exponential gradient in the flux distribution equivalent to the broad-band surface-brightness profile.

As an estimate for our error budget we take half the beam-smearing correction to be the uncertainty in the correction, noting that (a) this will tend to be an over-estimate; and (b) the correction and therefore the uncertainty are small. Beam-smearing corrections at $2.2 \mathrm{~h}_{R}$ are typically $4 \%$ for SparsePak and $1 \%$ for PPak. We adopt the larger value, namely $\sqrt{\left|\sigma_{\text {beam }}^{2}\right|} / \sigma_{\text {obs }}=0.04$, and $\Delta \ln \sigma_{\text {beam }}=0.5$ for the calculations below. Errors in the beamsmearing correction are systematic per fiber, but random over the average of a stack of fibers, therefore representing a random error in terms of budgeting.

\subsubsection{Line-of-sight integration}

There is also a broadening of $\sigma_{\text {LOS }}$ due to the line-of-sight integration through a differentially rotating disk of finite thickness. This occurs even for an infinitely small beam. While the cause is different than spatial beam-smearing, the effect is similar. For low-inclination disks, we might expect this effect to be negligible. The typical radial range, $\delta R$, of the lineof-sight through a disk in the DMS can be estimated based on the typical disk inclination $\left(\mathrm{i}=28^{\circ}\right)$ and disk radial scale length $\left(h_{R}=3.6 \mathrm{kpc}\right.$, or 12.6 arcsec $)$ of the Phase-B sample (Paper I). From this the disk oblateness can be estimated $\left(q_{R} \equiv h_{R} / h_{z} \sim 8 ; \S 2.2\right)$. The radial range can be approximated as $\delta R \sim 2 h_{z} \tan i=2 q_{R} h_{R} \tan i$. Hence $\delta R$ is typically 1.6 arcsec - substantially smaller than either the SparsePak or PPak fiber "beams." Since beam-smearing is a small effect in the DMS, we consider the effect of line-of-sight integration to be negligible.

\subsubsection{Line-of-sight projection}

The corrected velocity dispersion, $\sigma_{\mathrm{LOS}}$, is a projection, along the line-of-sight, of the SVE:

$$
\sigma_{\mathrm{LOS}}^{2}=\left(\sigma_{R}^{2} \sin ^{2} \theta+\sigma_{\theta}^{2} \cos ^{2} \theta\right) \sin ^{2} i+\sigma_{z}^{2} \cos ^{2} i
$$

where $i$ is the galaxy inclination relative to the observer, and $\theta$ is the azimuthal angle from the kinematic major axis in the plane of the galaxy. With definitions for the principal SVE 
ratios $\alpha$ and $\beta(\S 2.1)$, we further define

$$
\gamma \equiv 1+\frac{\tan ^{2} i}{\alpha^{2}}\left(\sin ^{2} \theta+\beta^{2} \cos ^{2} \theta\right)
$$

to write

$$
\sigma_{z}^{2}=\frac{\sigma_{\mathrm{LOS}}^{2}}{\gamma \cos ^{2} i}
$$

To apply the correction to the co-added spectra in each radial bin, operationally we take an azimuthal average of $\sigma_{\mathrm{LOS}}$ and not $\sigma_{\mathrm{LOS}}^{2}$. Hence equation (4) requires solving an elliptic integral of the second kind for $\sqrt{\gamma}$. However, at low inclination we adopt an excellent

approximation $\sqrt{4}$ such that $\sigma_{z} \approx \bar{\sigma}_{\mathrm{LOS}} / \sqrt{\bar{\gamma}} \cos i$, where $\bar{\sigma}_{\mathrm{LOS}}$ is the azimuthally-averaged $\sigma_{\mathrm{LOS}}$ and

$$
\bar{\gamma} \equiv \frac{1}{2 \pi} \int_{0}^{2 \pi} \gamma d \theta=1+\frac{\tan ^{2} i}{2 \alpha^{2}}\left(1+\beta^{2}\right) .
$$

Anticipating the development in $\S 5$ for $\Sigma_{\text {dyn }}$, we estimate the uncertainties in $\sigma_{z}^{2}$. From the discussion in $\S 2.1$, we adopt expected typical values and uncertainties of $\alpha=0.6 \pm 0.15$ (25\%), $\beta=0.7 \pm 0.04$ (5\%), and $i=30 \pm 3.5^{\circ}(12 \%)$. We find $\bar{\gamma} \cos ^{2} i=1.32 \pm 0.29(22 \%)$ and $\sigma_{z}^{2}=(0.76 \pm 0.17) \bar{\sigma}_{\text {LOS }}^{2}$ (see Appendix B). Uncertainties in $\bar{\sigma}_{\mathrm{LOS}}(\S 3.3,3.4)$, inclination and $\bar{\gamma}$ all enter into the error budget discussion in $\S 5$. Here, however we see that despite the uncertainties in $\alpha$ and $\beta$, in general the correction is small because the inclination is small and $\gamma$ is near unity. This means that uncertainties introduced by this correction are modest, which is a specific reason why we have chosen the nearly face-on regime. The variation in $\sqrt{\bar{\gamma}}-1=\sigma_{\mathrm{LOS}} / \sigma_{z} \cos i-1$ is illustrated in Figure 5 as a function of inclination and SVE shape $(0.4<\alpha<1.0, \alpha=\beta)$; systematic errors in disk mass due to deprojection should scale with this function.

\subsubsection{Extinction}

With the exception of the isothermal case, where $\sigma_{z}$ is independent of scale-height, dust extinction in the disk can modulate the observed value of $\sigma_{\mathrm{LOS}}$. Considering the face-on case, an exponential vertical mass distribution is the most extreme situation in the sense that the range of $\sigma_{z}$ from mid-plane to infinite height above the disk changes by a factor of $\sqrt{2}$, i.e.,

\footnotetext{
${ }^{4}$ The exact relation is given by $\sigma_{z}=\bar{\sigma}_{\operatorname{LOS}} / \frac{\cos i}{2 \pi} \int_{0}^{2 \pi} \gamma^{1 / 2} d \theta$. However, $\left(\int_{0}^{2 \pi} \sqrt{\gamma} d \theta\right)^{2} \approx \int_{0}^{2 \pi} \gamma d \theta$ is an approximation good to $<0.2 \%$ for $i<45^{\circ}$ and $\alpha=\beta=0.7$, and $<0.5 \%$ in the same inclination range for plausible values of $0.3<\alpha<1$ and $0.7<\beta<1$.
} 
about 41\%, increasing with height (see van der Kruit 1988, whose general development in the absence of extinction we elaborate on here). The effect of a dust layer, then, will be to censor the mid-plane region and raise the observed $\sigma_{z}$. The impact will depend on the total optical depth and the relative scale-height of dust and stars. For example, an infinitely thin dust layer at the mid-plane will have no effect on $\sigma_{z}$, regardless of optical depth. In the absence of dust, or in the infinitely-thin dust-layer case, the integrated $\sigma_{z}$ is $\sqrt{3 / 2}$ times the mid-plane value for an exponential vertical mass-density distribution of constant $\Upsilon$. In general, the observed $\sigma_{z}$ will increase in either of two situations: (1) for a finite-thickness dust-layer as the optical depth increases at a given dust-layer thickness relative to the stellar distribution; or (2) as the thickness of the dust layer increases at a fixed total optical depth through the disk.

A reasonable assumption is that the dust layer is comparable to the gas layer, and much smaller than the stellar scale-height for the old stars we are using as dynamical tracers. This is consistent with the observations of edge-on systems by Dalcanton et al. (2004) for fast-rotating disks like those in the DMS. Even in slow-rotating disks, which have thicker dust distributions, Seth et al. (2005) find the RGB population have scale-heights 1.5 to 3 times larger than the dust. While Howk \& Savage (1999) have shown, via unsharp imagemasking, that there are pronounced extra-planar dust structures in fast-rotating disks seen edge-on, there is good reason to believe these structures are associated with the energetics of star-formation in spiral arms (e.g., Kamphuis et al. 2007). The use of unsharp-masking, however, removes the median extinction level from the image so that the visual impression over-emphasizes the relative strength of the extra-planar versus the near-planar extinction. Inspection of the un-filtered images is qualitatively consistent with the multi-band radiativetransfer analysis (e.g., Xilouris et al. 1998): The overall extinction at large scale-heights is substantially lower than that near the mid-plane.

More debatable is the total, face-on optical depth. Work by Domingue, Keel \& White (2000), Boissier et al. (2004), Holwerda et al. (2005), and Tamura et al. (2009) all point to the clumpy nature of dust extinction in disks seen at low inclination. The different methods used in these studies each have their own substantial uncertainties, but there appears a consensus that dust is heavily concentrated toward spiral arms, with patchy effective extinction in the range $0.3<\mathrm{A}_{B}^{\mathrm{i}}<2.5 \mathrm{mag}$; the inter-arm regions are relatively thin $\left(\mathrm{A}_{B}^{\mathrm{i}}<0.1 \mathrm{mag}\right)$ and become thinner with increasing radius. Keel \& White (2001) estimate that for the disks in their sample of spirals "half the dust mass [is] contained in only $20 \%$ of the projected area and $95 \%-98 \%$ of the dust mass contained in half the area."

An essential problem with all of these observational studies is their inability to access the full 3D geometry of the stellar and dust distribution. This geometry, as shown by Calzetti 
et al. (1994), is necessary for modeling accurately the extinction as it pertains to different layers of the disk. Radiative-transfer modeling using clumpy stellar and dust distributions (e.g., Matthews \& Wood, 2001, for the low surface-brightness galaxy UGC 7321) are the path forward, but such models have not yet been fully developed for a wide range of galaxy types. However, some superb, multi-wavelength modeling using smooth dust distributions have been carried out by Xilouris et al. (1997, 1999). Their models have been applied to edge-on, intermediate-type spirals, otherwise typical of DMS galaxies. They find that the face-on optical depth is less than one, implying galaxies seen face-on would be nearly transparent. The extinction values are comparable to those found suitable for moderateinclination galaxies using a simpler, but physically similar model (Verheijen 2001).

To reconcile these results with the observational studies of clumpy dust distributions, in a follow-up study Misiriotis et al. (2000) have shown that from an edge-on perspective putting dust smoothly in an exponential disk is indistinguishable from placing the dust in a logarithmic spiral pattern of similar scale-height. Further, Popescu et al. (2000) and Misiriotis et al. (2001) examined whether the smooth dust-models of Xilouris et al. were consistent not only with the apparent extinction of star-light, but also the far-infrared (FIR) emission from the dust itself. Based on an FIR excess, they found evidence for an additional dust component, plausibly from a very thin layer associated with mid-plane star-formation.

The coherent picture emerging from all of the above discussion is one where the patchy regions of high extinction in spiral arms are associated with this nearly mid-plane dust responsible for the FIR excess, whereas the remainder of the disk is characterized better by the log-spiral dust-model of Misiriotis et al. (2000). Because regions of high extinction near the mid-plane do not effect $\sigma_{\mathrm{LOS}}$, we therefore adopt the smooth dust-distribution models of Xilouris et al. as a suitable mean for the arm-interarm disk extinction modulating $\sigma_{\mathrm{LOS}}$. In $\S 3.7$ we test our hypothetical picture by exploring if azimuthal variations in $\sigma_{\mathrm{LOS}}$ might be due to clumpy dust distributions associated with spiral structure.

Here, then, we characterize the dust to be in a smooth exponential distribution in radius and height. We adopt the full range of values from the work of Xilouris et al. (1997, $1998,1999)$ such that the dust has a scale-height of roughly $0.6 \pm 0.3$ that of the stars, an exponential radial scale-length of roughly $1.4 \pm 0.2$ that of the stars, and a face-on optical depths of $\tau=0.35 \pm 0.15$ and $0.55 \pm 0.20$ in the $I$ and $V$ bands, respectively, i.e., matching the Ca II-triplet and $\mathrm{Mg}$ Ib regions. We have computed the impact on the observed $\sigma_{z}$ in the worst-case scenario where $\tau=0.9$ (at the galaxy center), and the dust-to-star scale-height ratio is 0.9: This is the worst case scenario in the sense that both optical depth and dustto-star thickness are one standard deviation off their nominal values such that the impact on $\sigma_{z}$ is maximized. The change in $\sigma_{z}$ is only $1.3 \%$ higher from the dustless case. Since 
our measurements are actually made at around 1 dust scale-length, with typically smaller optical depths, the impact of extinction on the observed $\sigma_{z}$ is completely negligible. For this reason, we ignore extinction effects on $\sigma_{\mathrm{LOS}}$. The impact of extinction on the photometric aspect of $\Upsilon_{*}^{\text {disk }}$ is discussed below.

\subsubsection{Summary of corrections and uncertainties}

The final expression for the corrected vertical velocity dispersion of a stacked ring of fibers is given by

$$
\sigma_{z}^{2}=\bar{\sigma}_{\mathrm{LOS}}^{2} / \bar{\gamma} \cos ^{2} i
$$

where

$$
\bar{\sigma}_{\text {LOS }}^{2}=\bar{\sigma}_{\text {obs }}^{2}-\delta \bar{\sigma}_{\text {inst }}^{2}-\bar{\sigma}_{\text {beam }}^{2}
$$

$\bar{\sigma}_{\text {obs }}$ is the observed velocity dispersion from an averaged set of velocity-registered fibers in a ring; $\delta \bar{\sigma}_{\text {inst }}$ is the average correction for instrumental broadening not naturally taken out by the cross-correlation process $(\S 3.5 .1) ; \bar{\sigma}_{\text {beam }}$ is the average correction for beam smearing due to the finite size of our sampling aperture (§3.5.2); and $\bar{\gamma}$ is the correction for line-of-sight projection (§3.5.4). We have ignored corrections for line-of-sight integration (§3.5.3) and extinction (§3.5.5), and likewise the uncertainties in the error budget for $\sigma_{z}$ and quantities dependent upon $\sigma_{z}$.

The accounted uncertainties in $\sigma_{z}$ from measurements and corrections are listed in Table 1 in order: random measurement errors in $\sigma_{\mathrm{LOS}}$ from cross-correlation $\left(\Delta \ln \sigma_{\mathrm{obs}}, \S 3.3\right)$; random errors from template mismatch $\left(\Delta \ln \sigma_{\mathrm{tpl}}, \S 3.4\right)$; instrumental broadening mismatch between template and galaxy spectra $\left(\Delta \ln \sigma_{\text {inst }}, \S 3.5 .1\right)$; uncertainties in the beam-smearing correction $\left(\Delta \ln \sigma_{\text {beam }}, \S 3.5 .2\right)$; and errors in deprojection due to uncertainties in $\alpha, \beta$, and $i$ $(\S 3.5 .4)$.

\subsection{Other Considerations}

\subsubsection{Spatial registration}

IFU centering on a target galaxy can vary over a period of many consecutive hours of observation due to guiding errors or flexure. Such variations are small ( $<1$ arcsec), and

negligible over the course of a single exposure of 30-60 minutes. Larger centering variations can occur between observations of the same target taken on different nights or runs due to errors in offsets during target acquisition. We estimate the repeatability of target acquisition 
with the WIYN 3.5m telescope 5 at about 1 arcsec (RMS) in either RA or DEC. Offsets of $20-30 \%$ of a SparsePak fiber diameter are therefore typical of a set of observations of a single galaxy. For PPak observations, the position of a guide star on the guide CCD of the spectrograph is repeatable to 0.2 arcsec, i.e., the pixel size of the guide CCD, or $8 \%$ of the PPak fiber diameter.

Precise and accurate spatial registration of the IFU spectroscopy must be made both internally to the different spectroscopic pointings as well as externally to the imaging photometry. A robust determination of the relative spatial registration of the spectral data is critical for kinematic measurements. Uncorrected drifts, offsets, and mis-alignment of the pointing lead to an effective beam-smearing when fiber spectra are coadded, and to a mismatch when modeling the data or combining data across configurations (e.g., $\mathrm{H} \alpha$ with $\mathrm{Mg} \mathrm{Ib}$ and Ca II-triplet region data). Registration of the IFU spectroscopic data to the imaging photometry is required to match the kinematic measurements of enclosed mass and mass surface-density to enclosed luminosity and surface-brightness. Since the IFUs sparsely sample the spatial dimensions, it is all the more important that both the kinematic and photometric "footprints" are aligned in order to minimize random errors in matching mass to light. To prevent a substantial contribution to our error budget, we want the spatial mis-registration to be smaller than the seeing. We set a conservative upper-limit for spatial mis-registration such that it increases the effective smearing due to seeing by no more than $\sim 20 \%$ in a quadrature sense. This results in a mis-registration requirement for SparsePak of $\leq 0.5$ arcsec for the median seeing conditions ( 0.8 arcsec FWHM; $\$ 3.6 .2$ ), or about $10 \%$ of the SparsePak fiber diameter. For PPak, with median seeing of 1.4 arcsec, the mis-registration requirement is $\leq 0.8$ arcsec, or $30 \%$ of the fiber diameter.

We use two independent methods to determine the relative spatial offsets of our spectroscopic data from SparsePak. Both take full advantage of the IFS spatial coverage. The first method uses the velocity-field fitting technique described in Paper I which simultaneously solves for the barycenter, position angle, inclination, rotation curve, and the relative offsets of the individual spectroscopic pointings for a given source (Andersen 2001; Andersen \& Bershady in preparation). The registration is strictly relative, but with the assumption that the galaxy barycenter is co-spatial with the optical center of the galaxy, this method also provides an absolute registration. As we note below, this assumption can be checked by comparing the kinematic center to the center derived from a comparison of the spectral continuum distribution to broad-band images.

\footnotetext{
${ }^{5}$ The WIYN Observatory, a joint facility of the University of Wisconsin-Madison, Indiana University, Yale University, and the National Optical Astronomy Observatories.
} 
Application of this kinematic technique to the SparsePak data in the $\mathrm{H} \alpha$ and $\mathrm{Mg} \mathrm{Ib}$ regions for the 7 galaxies noted in $§ 3.4 .2$ (Westfall 2009) yields median centering errors of 0.2 arcsec in the $\mathrm{H} \alpha$ region and 0.5 arcsec in the $\mathrm{Mg}$ Ib region. (Exposure times were 15 minutes in $\mathrm{H} \alpha$ and 45 minutes in $\mathrm{Mg} \mathrm{Ib}$, typical for our survey.) The amplitude of these random errors scale directly with the product of the number of lines fit in each region (we simultaneously fit [N II] and [S II] lines in addition to $\mathrm{H} \alpha$, whereas in the $\mathrm{Mg}$ Ib-region we only fit the $[\mathrm{O}$ III $] \lambda 5007$ line) and the total number of fibers per pointing for which lineemission is detected.6 There is some indication that the registration using [O III] $] 5007$ is somewhat (up to 15\%) noisier than expected from this scaling, consistent with a velocity field that qualitatively looks more chaotic than that measured in the $\mathrm{H} \alpha$ region. Systematic errors can also be estimated by examining differences in the registration when fitting a range of rotation curve models, parameterized with two to three variables that control the steepness of the rise, peak velocity, and asymptotic behavior at large radius. For a set of models yielding comparable minimum $\chi^{2}$ values, we find that these systematic errors are 2 to 2.5 times smaller than the random errors. There is a hint that systematic errors become proportionately smaller as random errors become larger, suggesting that we are overestimating our systematic errors. This sample of 7 galaxies, six of which range from $24^{\circ}<i<38^{\circ}$, are typical of our Phase B sample in terms of quality of the velocity-field fit, inclination, and velocity-field regularity. We conclude that relative centering errors of 5-10\% (random) and $2-4 \%$ (systematic) of a fiber diameter are representative of this kinematic method, provided good $\mathrm{S} / \mathrm{N}$ data and a smooth velocity field.

The second registration method aims at matching the spectral continuum in the IFS data-cubes, in a least-squares sense, with photometry from broad-band images in a similar wavelength region. One challenge is to define a meaningful $\chi^{2}$ statistic, requiring a good definition of detector gain and error propagation when deriving the extracted spectral continuum signal. Seeing variations between image and spectra can also be problematic (§3.6.2). In some early data, there were also uncertainties in the IFU position-angle, which complicated the $\chi^{2}$ mapping. However, by comparing the kinematic position angle from the velocity-field fits to the photometric position angles ameliorates even these uncertainties.

Two approaches have been taken to this continuum-fitting method. The first method fits the spectral continuum data directly to aperture photometry of the broad-band images using the same relative two-dimensional foot-print pattern as the IFU, and apertures that match the fiber diameter. Using this approach, Swaters et al. (2003) were able to register the spectral continuum from SparsePak $\mathrm{H} \alpha$ observations of a low surface-brightness galaxy

\footnotetext{
${ }^{6}$ This product is typically 245 for an $\mathrm{H} \alpha$ region pointing and 40 for the $\mathrm{Mg} \mathrm{Ib}$ region.
} 
(DDO 39) to an $R$-band image with a centroid precision of 0.5 arcsec or better. This source is substantially irregular and has considerable foreground contamination from Galactic stars. Application of this method to regular galaxies of normal surface-brightness at higher Galactic latitude should be more precise. The second method matches the spectral continuum to a one-dimensional light-profile. Bershady et al. (2005) report continuum-fitting in the Ca IItriplet region using SparsePak to the $I$-band surface-brightness profile of UGC 6918 with a centroid precision of 0.25 arcsec or better. This precision is comparable to the velocity-field fitting method, and allows us to place the IFS observations on an astrometric scale without making any assumptions about how light traces mass.

In a separate paper (Andersen\& Bershady, in preparation) we have used DensePak H $\alpha$ data of a similar sample of 39 nearly face-on galaxies from Andersen et al. (2006) to directly compare centers derived with the kinematic and photometric methods described here. As noted in Paper I, 14 of these galaxies are in the DMS. The distribution of offset differences (RMS) based on this comparison is characterized by a mode of 0.35 arcsec, but a mean of 0.7 arcsec due to a tail that extends to 2 arcsec and contains $10 \%$ of the sample. This tail is likely due to the failure of one of the centering methods. For example, the distribution extremum is UGC 4614, a galaxy not in the DMS but with a $\mathrm{H} \alpha$ velocity field showing strong twisting associated with spiral arms. In this case the kinematic method is likely more suspect because it is based on a fit to an axisymmetric model. In general, galaxies with large asymmetries in their rotation curves ( $>10 \%$ in velocity) show systematically larger offset differences. By using both methods we are able to identify sources with discrepant centering and then, by inspection, determine which method is likely most problematic, thereby minimizing centering errors. The above mentioned mode and mean of the offset-difference distribution are equivalent, respectively, to centroiding uncertainties of 0.25 and 0.5 arcsec in both methods, consistent with our earlier estimates. Hence the registration precision we are able to achieve with either kinematic- or continuum-fitting methods is good enough that it does not enter the error budget. The centering uncertainties for PPak are expected to be significantly smaller given its 331 fibers, each with smaller diameter than SparsePak.

\subsubsection{Seeing variations}

Although the impact of seeing-mismatch between IFU and broad-band data on their spatial registration appears to be small for the large-fiber IFUs used in the DMS, the commensurate impact on mismatching "mass to light" is a different issue. With changes in seeing, the light sampled by a single fiber may not represent the same effective physical region as that of an identical photometric aperture, even if it is correctly placed on a broad- 
band image. The issue is comparable to deriving colors for sources in imaging data with variable seeing. For measuring colors, the recourse is either to determine the seeing in each image and degrade all images to the worst-case seeing (if the source profile is not known a priori), or if the profile is known (e.g., from stars), to carry out profile fitting on each source in each image, and take a comparable fraction of the total light from the derived profile fit to each image. Unfortunately, we do not know the intrinsic light profile sampled by each fiber, and for SparsePak we do not have estimates of the seeing conditions during the IFU observations. For PPak observations, the CCD image of each guide star exposure is stored and at our disposal. From each series of guide star images, we have reconstructed the effective seeing during each of the 60 minute PPak exposures (Martinsson et al., in preparation). The distribution of PPak seeing conditions (FWHM), suitably averaged over the full exposure times for each galaxy, has a mean and standard deviation of $1.4 \pm 0.4$ arcsec; $80 \%$ of the data was taken in conditions between 0.9 and 2.0 arcsec; and all of the data was taken in conditions between 0.8 and 2.1 arcsec.

There are several factors which mitigate the impact of seeing mismatch. First, SparsePak fiber diameters are 6 times larger than the FWHM of the point-spread-function (PSF) in median conditions at WIYN (0.8 arcsec), so that $70 \%$ of the light collecting area of a fiber is more than the FWHM away from the fiber edge. This diminishes the impact of any unresolved sources in or near the edges of a fiber on the encircled energy as the PSF varies. Second, by employing the spectral-continuum registration method using an image with sufficiently good seeing, we can degrade the image quality until the best match is found between

spectral and imaging data in a $\chi^{2}$ sense (e.g., Andersen et al. 2008). Finally in the case of fiber averaging, either in spatially-adjacent regions or in an azimuthal ring, the impact of seeing variations will be averaged out. These mitigating factors, while only qualitatively described here, are sufficient to ignore seeing mismatch in further discussion of our error budget.

\subsection{Assessment of Uncorrected Systematics}

The accuracy of any measurement ultimately depends on the completeness of the assessment of, and correction for, systematic errors. The presence of overlooked or mis-estimated systematic effects abound in astronomy because of the complexity of astrophysical systems and the lack of direct laboratory verification. The troubled history of the extragalactic distance-scale measurement is but one notorious example. Since galaxies are complex systems, and the measurement of disk-mass involves a delicate confluence of photometric and kinematic scales, it would not be surprising to find that in ten years time our knowledge has 
increased sufficiently to revise the correction estimates presented in this paper. Nonetheless, at this time we do have sufficient information to surmise that any such revisions will likely be modest. Of the two primary components of the disk-mass measurement, $h_{z}$ and $\sigma_{z}$, we we have explored potential systematics associated with the vertical scale height extensively in $§ 2.2$. Here we present a simple inquiry on the likely amplitude of systematics in the stellar velocity dispersion.

A primary concern we have is that our approach relies on the assumption that to a high degree disks are axisymmetric and their mass-density distribution is smooth. Yet we know non-axisymmetric structure in the form of spiral arms, lopsidedness or elongation (bi-symmetry) are also salient features of disk systems. Specific concerns include: patchy extinction of unknown scale-height (raising $\sigma_{\mathrm{LOS}}$ due to mid-plane censoring of an exponential vertical mass-distribution); population gradients (modulating $\sigma_{\text {LOS }}$ due to template mismatch and $\sigma-h_{z}$ trends with age); and velocity structure (enhancing $\sigma_{\mathrm{LOS}}$ due to beam smearing from, e.g., streaming motions in spiral arms). To check for these effects we have picked UGC 6918 as a test case because it has the highest surface-brightness disk in our sample (and hence is most likely to have significant extinction at least in its inner regions); it has among the most asymmetric rotation curves in our sample (Andersen et al. in preparation); and it contains a weak AGN - activity perhaps resulting from the perturbed kinematics and also plausibly modulating stellar-population gradients.

In Figure 15 we illustrate residuals in the spectral continuum, stellar velocity and stellar velocity dispersion from a smooth, axisymmetric model. Kinematic measurements have been made for individual fibers using a single (K1 III) stellar template so that we may maximize our sensitivity to both radial and azimuthal variations. We have constructed the residuals from an axusymmetric model in the simplest and least parametric way possible. The model consists of independent Legendre polynomial fits of low order to the radial distributions of each of these quantities. For purposes of illustration the velocity data is deprojected for azimuthal location but not inclination, and we exclude from the fit point that are more than $60^{\circ}$ from the kinematic major axis. However, the velocity residuals are calculated from the derived rotation curve projected in azimuth. Similarly, the model fit to the radial trend of the stellar velocity dispersion is modulated in azimuth, when calculating residuals, by an SVE with $\alpha=0.6$ (expected to be typical of late-type disks) and $\beta$ set by the epicycle approximation and the model rotation curve.

By construction the residuals in radius have no structure, although there is scatter at smaller radii substantially in excess of the small estimated measurement errors. The scatter is below $12 \% \mathrm{rms}$ in surface-brightness and $\sigma_{\mathrm{LOS}}$, and roughly $3 \mathrm{~km} \mathrm{~s}^{-1} \mathrm{rms}$ in velocity. In azimuth, however there is clearly correlated structure, most evident in the surface-brightness 
and $\sigma_{\mathrm{LOS}}$ residuals. The amplitude of this structure is below $15 \%$. One half of the galaxy also appears to be rotating $10 \mathrm{~km}^{-1}$ faster than the other (in projection).

The bottom panels of Figure 15 explore whether variations in $\sigma_{\mathrm{LOS}}$ are correlated with variations in surface-brightness (as might be expected if there is patchy extinction associated with spiral structure) or deviations from smooth rotation. There is no evident correlation between any of these quantities.

From this analysis we conclude that in the worst possible case (a high surface-brightness, kinematically asymmetric disk) systematics are below $15 \%$, and even these amplitude are only apparent at the innermost radii where extinction is likely greatest. Our expectations are that for the remainder of our survey sample, such systematics will be smaller still, and hence negligible to our overall error budget.

\section{DISTANCE ERRORS}

The flow-corrected distances, $D$, given in Table 2 of Paper I have $7 \pm 0.5 \%$ formal errors, which are the quadrature sum of the estimated uncertainty in $H_{0}$ (NED7 quotes $73 \pm 5$, or $\Delta \ln D_{\mathrm{H} 0}=0.07$; cf. Mould et al. (2000), who estimate 9\%) and the heliocentric velocity measurements. The latter are insignificant. Yet flow corrections are large, and their precision and accuracy should be somewhat suspect at least to the extent that there are no errors associated with these corrections attributed to the distance-estimates. Uncertainties arise both from the flow-model as well as peculiar velocities on top of any bulk flow. For example, for both Phase-A and Phase-B samples, the median flow-correction $\Delta V_{\text {flow }} \equiv V_{\text {hel }}-73 D$ is $\sim-300 \mathrm{~km} \mathrm{~s}^{-1}$. Flow corrections range from $-811 \mathrm{~km} \mathrm{~s}^{-1}$ to $+1301 \mathrm{~km} \mathrm{~s}^{-1} ; 90 \%$ of the sample is contained within $-620<\Delta V_{\text {flow }}<+230 \mathrm{~km} \mathrm{~s}^{-1}$. These values are fairly independent of heliocentric velocity, which means the corrections become very large at small recession velocities. The largest corrections are in the sense of lowering distances and related distance-dependent quantities of luminosity and size, but raising $\Sigma_{\text {dyn }}$ and $\Upsilon$. However the median correction is to increase distances, and hence the predominate effect of flowcorrections is to raise luminosity and size estimates, but diminish $\Sigma_{\text {dyn }}$ and $\Upsilon$.

Taking the quantity $\Delta \ln D_{\text {flow }} \equiv\left|0.5\left[1-V_{\text {hel }} /(73 \times D)\right]\right|$ as a conservative distance-error statistic associated with flow-corrections and peculiar velocities, we find $90 \%$ of the Phase-A and Phase-B samples have distances errors $<11 \%$ and $<8 \%$ respectively; $75 \%$ of the samples have distances errors $<6 \%$ and $<4 \%$; and $50 \%$ of the samples have distances errors $<3 \%$

\footnotetext{
${ }^{7}$ NASA/IPAC Extragalactic Database: http://nedwww.ipac.caltech.edu/
} 
and $<2 \%$. Similarly, $\Delta \ln D_{\text {flow }}$ is less than $10 \%$ for $V_{\text {hel }}>2100 \mathrm{~km} \mathrm{~s}^{-1}$ and less than $5 \%$ for $V_{\text {hel }}>5000 \mathrm{~km} \mathrm{~s}^{-1}$ for the Phase-A sample, and $\Delta \ln D_{\text {flow }}$ is less than $10 \%$ for $V_{\text {hel }}>1450$ $\mathrm{km} \mathrm{s}^{-1}$ and less than $5 \%$ for $V_{\text {hel }}>3350 \mathrm{~km} \mathrm{~s}^{-1}$ for the Phase-B sample. Adopting the 90th percentile errors for $\Delta \ln D_{\text {flow }}$ for the Phase-B sample in quadrature with uncertainties in $H_{0}$, we arrive at a total systematic error of $11 \%$ for distance. This is applicable to individual galaxies. Since sources cover a range of distances and directions in the sky, velocity-flow corrections to their distances introduce random errors in the ensemble measurements. For the survey as a whole, the systematics are diminished to the uncertainty in $\mathrm{H}_{0}$ only, or $7 \%$.

In future papers we discuss some individual cases, e.g., UGC 6918, where other indicators such as super-novae can be used to better-calibrate distances. Of course, TF can be used too, but since its use inherently biases the luminosity estimate on a case-by-case basis (although not necessarily in the mean), wherever possible we prefer to avoid this approach. This is necessarily the case when using iTF to infer inclinations.

\section{SURVEY ERROR BUDGET}

The formulae for the dynamical disk mass, $\Sigma_{\text {dyn }}$, the disk stellar mass-to-light ratio, $\Upsilon_{*}^{\text {disk }}$, the disk fraction, $\mathcal{F}_{* \text {,max }}^{\text {disk }}$, and the baryon fraction, $\mathcal{F}_{\text {bar }}$ are presented in terms of our observables. These formulae are used to derive our error budget for each quantity, and to identify which portions of this budget lead to systematic or random uncertainties for individual galaxies and for the survey as a whole. For illustration purposes, we compute the errors on the disk-averaged values $\left\langle\Sigma_{\text {dyn }}\right\rangle$ and $\left\langle\Upsilon_{*}^{\text {disk }}\right\rangle$. We make the canonical distinction between "random" and "systematic" error components to mean that if the observation were repeated under identical conditions, random errors lead to a variance in the derived value. Systematic errors in individual galaxy measurements which scatter symmetrically around an accurate value of the mean for the sample ensemble, are categorized as random uncertainties for the survey.

\subsection{Disk Dynamical Mass, $\Sigma_{\text {dyn }}$}

As a nominal model we will adopt an exponential vertical distribution function $(k=3 / 2)$ of constant scale-height and oblateness as parameterized in $\S 2.2$ by equation (1). Scaled to our nominal model, physical units and characteristic values expected for the DMS, the radial 
expression for dynamical mass surface-density becomes

$$
\Sigma_{\mathrm{dyn}}(R)\left[\mathcal{M}_{\odot} \mathrm{pc}^{-2}\right]=100.0\left(\frac{k}{3 / 2}\right)^{-1}\left(\frac{h_{z}}{444 \mathrm{pc}}\right)^{-1}\left(\frac{\sigma_{z}(R)}{30 \mathrm{~km} \mathrm{~s}^{-1}}\right)^{2}
$$

Substitute $h_{R}$ for $h_{z}, \Sigma_{\text {dyn }}$ can be written as an explicit function of our observables:

$$
\begin{aligned}
& \Sigma_{\mathrm{dyn}}(R)\left[\mathcal{M}_{\odot} \mathrm{pc}^{-2}\right]= \\
& 100\left(\frac{k}{3 / 2}\right)^{-1}\left(\frac{q}{q_{R}}\right)\left[\left(\frac{D}{60.1 \mathrm{Mpc}}\right)\left(\frac{h_{R}}{12 .{ }^{\prime \prime} 6}\right)\right]^{-0.63}\left(\frac{\sigma_{z}(R)}{30 \mathrm{~km} \mathrm{~s}^{-1}}\right)^{2},
\end{aligned}
$$

where $q$ is the true axis ratio (oblateness) $h_{R} / h_{z}$, and $q_{R}$ is the estimate given in equation 1 .

Equation (9) becomes our operating formula for estimating errors. The first two quantities $(k$ and $q)$ are unity for the nominal model, and have (known) uncertainties $(\Delta \ln k=0.14$ and $\left.\Delta \ln q=\Delta \ln q_{R}=0.25\right)$. They lead to systematic errors for individual galaxies, but random errors over the survey as a whole. For our calculation here, we assume that galaxies do not deviate systematically from our adopted oblateness relation and our assumed form for the vertical density distribution. The last three parenthetical quantities in equation (9) are our observables. In order: Distance uncertainties, discussed in $\S 4$ above, are systematic for individual galaxies, and contain systematic $\left(\mathrm{H}_{0}\right)$ and random (flow) errors for the survey as a whole. Random errors in the measured recession velocities are negligible. Measurement uncertainties in $h_{R}$ are random. We note the reduced distance- and size-dependence due to the correlation of oblateness with galaxy scale. Errors in $\sigma_{z}$ are random, including the observed line-of-sight dispersion, beam-smearing and instrumental corrections, inclination, template-mismatch, and the SVE shape. We assume in our model that estimates for the SVE shape and its uncertainty are made on a galaxy-by-galaxy basis. Survey systematic errors for $\Sigma_{\text {dyn }}$, then, include only distance errors from $\mathrm{H}_{0}$.

Several caveats are worth mentioning regarding the impact on our $\Sigma_{\text {dyn }}$ estimate due to the presence of (i) a radial gradient in the halo potential, (ii) a thick disk, and (iii) a thin gas-layer. First, the impact of the halo potential on $\sigma_{z}$ should be small because the halo density gradient is locally negligible compared to the radial density gradient of the disk, particularly in the radial region of interest in our survey. Both (ii) and (iii) alter the effective $k$ value that should be used with the associated $h_{z}$ and $\sigma_{z}$, and also the effective value of $h_{z}$. Since the effect of a thick disk is to make $h_{z}$ larger and $k$ smaller (a two-component monotonic profile is always cuspier than a one-component monotonic profile), the effects of a thick disk on our estimates of $\Sigma_{\text {dyn }}$ tend to cancel. We also demonstrated in $\S 2.2$ that the impact of a thick disk on the effective value of $h_{z}$ is smaller than the plausible range of $k$. Hence we include the estimate of $\Delta \ln k$ alone as a parenthetical entry in Table 1 to indicate 
an upper limit to the potential systematics associated with variations in the effective vertical density-distribution.

Appendix B.1 has the formulation of the random and systematic errors for $\left\langle\Sigma_{\mathrm{dyn}}\right\rangle$ under the plausible model that the observables are independent. Table 1 collects the terms in three categories: (i) measurement errors on $\sigma_{\text {obs }}$ (§3.3) and $h_{R}(\S 2.2)$; (ii) uncertainties from correcting for systematic effects, including instrumental resolution (§3.5.1), beam smearing (§3.5.2), line-of-sight projection (§3.5.4), template-mismatch (§3.4), and oblateness (§2.2); and (iii) uncorrected systematic effects, which include the vertical distribution function and distance $(\S 4)$. We have excluded the effects on $\sigma_{\text {LOS }}$ from line-of-sight integration (§3.5.3) and dust extinction (§3.5.5) as negligible contributions to the error budget. A typical survey value of $\sigma_{z}=30 \mathrm{~km} \mathrm{~s}^{-1}$ and $i=30^{\circ}$ are adopted to set the error scale. Results depend weakly on the choice of $\sigma_{z}$ because the dominant errors (deprojection and template-mismatch) scale. However, there is a significant dependence to the error in $\Sigma_{\text {dyn }}$ on inclination, illustrated in Figure 16.

Typical errors for individual galaxies in our survey will be $28 \%$ (ran) and $26 \%$ (sys) for $\Sigma_{\text {dyn }}$. The dominant error terms for $\Sigma_{\text {dyn }}$ are (i) the conversion of scale-length to scaleheight (25\% systematic error); and (ii) the deprojection of $\sigma_{\mathrm{LOS}}$ into $\sigma_{z}$ (27\% random error). Contributions to the latter come nearly equally from uncertainties in the ellipsoid ratio, $\alpha$, and the inclination. Dividing the sample into quartiles (e.g., 10 galaxies binned by color, luminosity, or surface-brightness) will yield errors of $12 \%$ (ran) and $4 \%$ (sys) for $\Sigma_{\text {dyn }}$.

\subsection{Disk Stellar Mass, $\Sigma_{*}$, and Mass-to-Light Ratio, $\Upsilon_{*}^{\text {disk }}$}

The total dynamical disk M/L can be written as $\Upsilon_{\text {dyn }}^{\text {disk }}=\Sigma_{\text {dyn }} / I$, where the surfaceintensity $I$ has units of $L_{\odot} \mathrm{pc}^{-2}$, and it is implicit that $I$ is associated with the disk. This is an empirical definition based on the apparent luminosity distribution. It is useful for mass-decompositions when information on other constituents of the disk (gas and dust) is

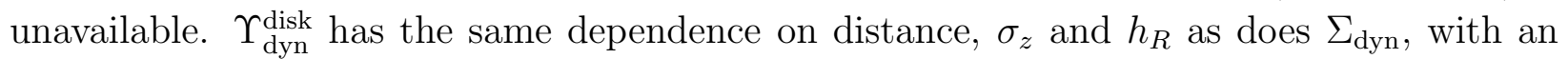
added dependence on the surface-photometry. The latter in general is not a limiting factor in terms of random error.

To obtain $\Upsilon_{*}^{\text {disk}}$, however, requires an estimate of the stellar mass surface-density, $\Sigma_{*}$, and the extinction-corrected flux surface-intensity, $I^{c}$ : $\Upsilon_{*}^{\text {disk }}=\Sigma_{*} / I^{c}$. We express the flux surface-intensity in wavelength-specific form where $I_{\lambda}=I_{\lambda}^{c} \exp \left(-\tau_{\lambda}\right)$, and the extinction in magnitudes, $\mathrm{A}_{\lambda}=1.086 \tau_{\lambda}=-2.5 \log \left(I_{\lambda} / I_{\lambda}^{c}\right)=\mu_{\lambda}-\mu_{\lambda}^{c} ; \mu$ is equivalent to $I$, except expressed in mag $\operatorname{arcsec}^{-2}$. The extinction correction is the sum of the Galactic foreground 
extinction $A_{\lambda}^{g}$ and effective internal extinction $A_{\lambda}^{i}$ within the galaxy, given the complexities of the dust geometry, scattering, and (at long wavelengths) emissivity. Internal extinction is modest in the outer parts of late-type disks (§3.5.5), and for our reddest near-infrared bands ( $K$ and $3.6 \mu \mathrm{m}$ Spitzer bands) extinction corrections are small, as are the uncertainties in these corrections. There is also a $\sim 3 \%$ zero-point uncertainty inherent to any magnitude system (e.g., the uncertainties in the $A B_{95}$ calibration; Fukugita et al. 1996).

To arrive at $\Sigma_{*}$ corrections must be made for the gas mass surface-density $\left(\Sigma_{\text {gas }}\right)$, comprised of atomic $\left(\Sigma_{\text {atom }}\right)$ and molecular $\left(\Sigma_{\text {mol }}\right)$ components, and for non-baryonic disk dark matter $\left(\Sigma_{\text {dark }}\right)$. We ignore ionized gas and dust contributions to $\Sigma_{\text {dyn }}$, assuming they are negligible. While we mention $\Sigma_{\text {dark }}$ here for completeness, we drop it from subsequent consideration; henceforth any non-baryonic disk component is subsumed in the stellar component, and we write: $\Sigma_{*}=\Sigma_{\text {dyn }}-\Sigma_{\text {atom }}-\Sigma_{\text {mol }}$.

Measurements of the neutral hydrogen gas mass surface-density $\left(\Sigma_{\mathrm{HI}}\right)$ is straightforward and part of our program. We scale $\Sigma_{\mathrm{HI}}$ by a factor of 1.4 to arrive at the total atomic gasdensity. This scaling takes nucleosynthesis products into account (a factor of 1.32 corresponds to a $24 \%$ primordial helium mass-fraction). Estimating $\Sigma_{\text {mol }}$ is more indirect and uncertain, although Spitzer images at 8, 24, and $70 \mu \mathrm{m}$ are at our disposal; flux-ratios from these bands imply dust of various grain sizes, and indirectly molecular gas phase.

First, we adopt $\eta \equiv \mathcal{M}_{\text {mol }} / \mathcal{M}_{\text {atom }}$ as the parameterization the total mass ratio of molecular to atomic gas, and we assume the atomic and molecular phases have comparable abundances. Early studies by Young \& Knezek (1989) based on measurements of CO(1-0) lineemission indicated $\eta$ was of order unity, but varied strongly with Hubble type between 1.8 to 0.3 going from $\mathrm{Sb}$ to Scd. They found the range for a given type comparable to the mean. More recent work by Casoli et al. (1998) shows $\eta<1$ for all types decreasing from roughly 0.35 to 0.1 between $\mathrm{Sb}$ and $\mathrm{Sd}$, although this trend is reduced if only the most dynamically massive systems are considered. This result is consistent with the most recent literature-compilation studies, e.g., Obreschkow \& Rawlings (2009). We adopt a values of $\eta=0.25 \pm 0.1(\Delta \ln \eta=0.4)$ as typical for galaxies in our survey. Since the atomic mass density contributes roughly $11 \pm 6 \%$ of the total dynamical mass within the luminous portion of a typical intermediate-type disk (e.g., Verheijen 1997; Hoekstra et al. 2001), the molecular component is of order $3 \pm 2 \%$. Uncertainty in the mass-contribution of the molecular component can be further reduced by accounting for the observed correlations of $\eta$ with farinfrared luminosity and dynamical mass, as noted by Casoli et al. (1998). More recent work by Leroy et al. (2008) indicates even more precise estimates of the distribution of molecular gas mass can be made via $24 \mu \mathrm{m}$ flux maps, which we have for our entire sample.

Second, to arrive at $\Sigma_{\text {mol }}$ we adopt a second parameterization, namely $\xi \equiv \Sigma_{\mathrm{mol}} /\left(\Sigma_{*}+\right.$ 
$\Sigma_{\text {mol }}$ ), and make the reasonable approximation that while the atomic and molecular total masses are correlated, the mass surface-density of the molecular component is better correlated with the stellar mass surface-density (Regan et al. 2001). This approximation allows us to substitute masses for mass surface-densities in the definition of $\xi$. Taking the sum of atomic and molecular gas along with stars as the total dynamical disk mass, $\mathcal{M}_{\text {dyn }}^{\text {disk }}$, we can then rewrite $\xi=\eta /\left(\mathcal{M}_{\mathrm{dyn}}^{\text {disk }} / 1.4 \mathcal{M}_{\mathrm{HI}}-1\right)$, which is a constant estimated for each galaxy. From this it follows $\Sigma_{*}=(1-\xi)\left(\Sigma_{\mathrm{dyn}}-1.4 \Sigma_{\mathrm{HI}}\right)$. Finally, the expression for the stellar M/L at a given wavelength and radius in the disk,

$$
\Upsilon_{*, \lambda}^{\text {disk }}(R)=\frac{(1-\xi)\left[\Sigma_{\mathrm{dyn}}(R)-1.4 \Sigma_{\mathrm{HI}}(R)\right]}{\operatorname{dexp}\left[-0.4\left(\mu_{\lambda}(R)-\mathrm{A}_{\lambda}(R)-M_{\odot, \lambda}-21.57\right)\right]},
$$

becomes a function of our observables and parameterization of the molecular mass component. In this equation, $\Upsilon_{*, \lambda}^{\text {disk }}$ has solar units when the $\Sigma$ 's are expressed in $\mathcal{M}_{\odot} \mathrm{pc}^{-2}$. The luminosity scale is set by $M_{\odot, \lambda}-21.57 \equiv \mu_{\odot, \lambda}$, where $M_{\odot, \lambda}$ is the Solar absolute magnitude in the relevant band and magnitude system. Appendix B.2 contains the formulation of the random and systematic errors for $\Sigma_{*}$ and $\Upsilon_{*, \lambda}^{\text {disk }}$.

To set the error-scale, a number of values need to be defined. In terms of masses, first we assign a $3 \%$ random measurement error to $\Sigma_{\mathrm{HI}}$ in a given radial ring $(2.5 \%$ flux-calibration error, $2 \%$ random error given $\mathrm{S} / \mathrm{N} \sim 15$ per beam and typically 10 beams per ring). Second, we assign typical values of $N_{\text {disk }}=5$ and $N_{\text {atom }}=10$ as the number of radial bins in the stellar $\sigma_{\mathrm{LOS}}$ and H I maps, respectively, to relate errors in total disk and H I mass to their respective surface-density errors. Third, we estimate a characteristic ratio of the stellar to H I disks masses, $\mathcal{M}_{*}^{\text {disk }} / \mathcal{M}_{\mathrm{HI}}$, to be $7.2 \pm 3.8$ for a maximum-disk, or $3.5 \pm 1.9$ for a socalled "Bottema-disk" (Bottema 1997) 8 based on the 13 Sb-Scd galaxies from the study by Hoekstra et al. (2001); we adopt the mean Bottema-disk value here. From these values and given $\eta$ above, it follows that $\xi=0.09$. The uncertainty in $\xi$ is large $(40 \%$ random error and $53 \%$ systematic error at $i=30^{\circ}$ ) due to a combination of the uncertainty in the moleculargas mass and the measurement errors in the atomic and total disk masses. However, what is relevant to the error in $\Sigma_{*}$ and $\Upsilon_{*}^{\text {disk }}$ is the quantity $[\xi /(1-\xi)] \Delta \ln \eta$, which is only about $4 \%$. Finally, we adopt the mean value $\Sigma_{*} / \Sigma_{\mathrm{HI}}=9.0 \pm 5.4$ for a Bottema-disk, which follows from $\mathcal{M}_{*}^{\text {disk }} / \mathcal{M}_{\mathrm{HI}}$ above, and characteristic radii $R_{\mathrm{HI}} / R_{*} \sim 1.6 \pm 0.3$ based on Verheijen \& Sancisi (2001). The variances quoted here for $\mathcal{M}_{*}^{\text {disk }} / \mathcal{M}_{\mathrm{HI}}$ and $R_{\mathrm{HI}} / R_{*}$ are astrophysical and do not enter into our error estimates because they are measurable quantities in our survey. For estimating characteristic errors of individual galaxies or ensemble averages we adopt the mean values for $M_{*} / \mathcal{M}_{\mathrm{HI}}$ and $\Sigma_{*} / \Sigma_{\mathrm{HI}}$. Note that $\Sigma_{*}$ and $\Sigma_{\mathrm{HI}}$ have very different radial dependencies.

\footnotetext{
${ }^{8} \mathrm{~A}$ so-called Bottema-disk has $\mathcal{F}_{*, \text { max }}^{\text {disk }}=0.63$, whereas a maximum disk has $0.85<\mathcal{F}_{*, \text { max }}^{\text {disk }}<0.90$.
} 
For photometric errors we adopt $\Delta \mu_{\lambda}=0.05 \mathrm{mag} \operatorname{arcsec}^{-2}$ as a conservative upper-limit to both photon shot-noise and calibration uncertainty. Adopting the model in Verheijen (2001), we find $\mathrm{A}_{\lambda}^{\mathrm{i}}=0.45,0.15,0.02 \mathrm{mag}$ in the $B, I, K$ bands, respectively. As an upper limit, we take $\Delta \mathrm{A}_{\lambda}=0.4 \mathrm{~A}_{\lambda}^{\mathrm{i}}$ based on the variance in the Xilouris et al. studies, and assume that this uncertainty dominates over uncertainties in correction for foreground extinction (we selected galaxies with $\mathrm{A}_{B}^{\mathrm{g}}<0.25 \mathrm{mag}$; Paper I). Treated in this way, correction for extinction introduces a random error assuming extinction variations across a disk. In practice, measurement of the broad-band spectral energy distributions (SEDs), the Balmer decrement, or the $\mathrm{H} \alpha$ to $24 \mu \mathrm{m}$ flux ratio will allow us to estimate the extinction on a spatially resolved scale, thereby reducing this uncertainty. Possible exceptions are regions of high-extinction in spiral arms, which we will be able to identify via Spitzer 8, 24, and $70 \mu$ maps.

Appendix B.2 has the formulation of the random and systematic errors for $\Sigma_{*}$ and $\Upsilon_{*}^{\text {disk }}$ under the plausible model that the observables are independent. Table 2 collects the terms including (i) measurement errors on $\mu_{\lambda}$ and $\Sigma_{\mathrm{HI}}$, and (ii) uncertainties from correcting for systematic effects, which include extinction and the molecular mass-fraction. The inclination dependence of errors in $\Upsilon_{*, K}^{\text {disk }}$ are illustrated in Figure 15. We have excluded spatial registration errors (§3.6.1) and seeing variations (§3.6.2) as negligible contributions to the error budget. The error on $\Sigma_{\text {dyn }}$ is brought forward from Table 1. There are no additional uncorrected systematic effects. With the exception of the molecular gas-mass fraction, parameterized by $\eta$, and systematic effects in $\Sigma_{\text {dyn }}$, the remaining uncertainties introduce random errors on individual $\Upsilon_{*}^{\text {disk }}$ measurements in any one galaxy. For example, an error in $\mu_{\lambda}^{c}$ is a random error for $\Upsilon_{*}^{\text {disk }}$ because the $\mu_{\lambda}$ measurement-error is random, and $\Delta A_{\lambda}$ is treatable as a random error (extinction variations from galaxy to galaxy and within a galaxy are stochastic but estimable). Ensemble estimates of $\Upsilon_{*}^{\text {disk }}$ for the survey as a whole suffer only from systematic errors as described for $\Sigma_{\text {dyn }}$. Typical errors in $\Upsilon_{*}^{\text {disk }}$ for individual galaxies in our survey will be $32 \%$ (ran) and 30\% (sys), weakly dependent on band-pass. Dividing the sample into quartiles will lower $\Upsilon_{*}^{\text {disk }}$ errors to $14 \%$ (ran) and $6 \%$ (sys).

\subsection{Disk fraction, $\mathcal{F}_{*, \max }^{\text {disk }}$}

The disk mass fraction within some radius, $R$, is defined as

$$
\left.\mathcal{F}_{* \text { max }}^{\text {disk }}(R) \equiv \frac{V_{*}^{\text {disk }}}{V_{\mathrm{c}}}\right|_{R}
$$

where $V_{*}^{\text {disk }}$ and $V_{\mathrm{c}}$ are the circular rotation speeds associated with the disk stars and entire potential, respectively. Operationally, we substitute $V_{*}^{\text {disk }}=\sqrt{f_{*}^{\text {disk}} \mathcal{M}_{*}^{\text {disk }} \mathrm{G} / R}$, and adopt the disk gas tangential speed for $V_{\mathrm{c}} ; V_{\mathrm{c}}, V_{*}^{\text {disk }}$ and $\mathcal{M}_{*}^{\text {disk }}$ are all functions of $R$. The factor $f_{*}^{\text {disk }}$ 
accounts for the non-sphericity of the mass-distribution in the potential symmetry-plane. In general, $f_{i}$ for the $i^{\text {th }}$ mass-component can be a function of radius, but for purposes here we assume the disk oblateness, and hence $f_{*}^{\text {disk }}$, is constant with radius.

$\mathcal{F}_{* \text {,disk }}$ takes on the value $\mathcal{F}_{* \text {,max }}^{\text {disk }}$ at $R=R_{* \text {,disk }}^{\max }$ where the rotation curve from the stellar disk component reaches a maximum, (i.e., $\left.V_{*}^{\text {disk }}=V_{*, \max }^{\text {disk }}\right)$. Using the numerical integration code of Casertano (1983), we find that $R_{* \text {,disk }}^{\max } \sim(2.2+1 / q) h_{R}$ for an oblate, constant $\Upsilon$ disk with $q>4$. Based on equation (1), $90 \%$ of our sample with stellar kinematic measurements has $0.09<1 / q_{R}<0.18$. We continue to assume that $q=q_{R}$ is a good statistical approximation.

We arrive at an estimate for $f_{*}^{\text {disk }}$ using our knowledge of the likely range of disk oblateness. Binney \& Tremaine (1987) illustrate that an infinitely thin $\left(h_{z} / h_{R}=0\right)$ exponential disk has a $15 \%$ higher peak velocity than its spherical counterpart $\left(V_{\text {disk }} / V_{\text {sphere }} \sim 1.15\right)$. Using numerical integration, we find $V_{\text {disk }} / V_{\text {sphere }} \sim 1.16-0.3 / q$ for $q>4$, illustrated in Figure 17. The mean disk oblateness of our sample is 0.14 . With a $25 \%$ uncertainty in disk oblateness for any individual galaxy, $f_{*}^{\text {disk }}=1.242 \pm 0.023$. This is less than a $1 \%$ contribution to the error budget of $f_{*}^{\text {disk }}$.

Appendix B.3 contains the error formulation for $\mathcal{F}_{* \text { max }}^{\text {disk }}$ in terms of the observational uncertainties associated with $V_{\mathrm{c}}$ and $\mathcal{M}_{*}^{\text {disk }}$ (which, as argued in the previous section is proportional to $\Delta \ln \Sigma_{\text {dyn }}$ ), and $f_{*}^{\text {disk }}$. In estimating errors on $V_{\mathrm{c}}$ we make the reasonable assumptions that velocity measurements of the gas are made; asymmetric drift is negligible such that $V_{\mathrm{c}}=V_{\text {rot }}$ is an excellent approximation; $V_{\text {rot }}=\left(V_{\text {obs }}-V_{\text {sys }}\right) / \sin i$ along the major axis, namely corrections for line-of-sight integration are negligible in nearly face-on disks; and errors on $V_{\text {sys }}$ are negligible. The terms are collected in Table 3. The inclination dependence of errors in $\mathcal{F}_{* \text {,max }}^{\text {disk }}$ are shown in Figure 16. Even though $f_{*}^{\text {disk }}$ is fundamentally related to $h_{z} / h_{R}$, it enters independently in $\mathcal{F}_{*, \max }^{\text {disk }}$ in addition to the impact of $h_{z} / h_{R}$ directly on $\Sigma_{\text {dyn }}$; uncertainty in $f_{*}^{\text {disk }}$ introduces systematic error for and individual galaxy. We exclude errors on $R_{* \text {,disk }}^{\max }$ because both the uncertainty in $h_{R}$ and range of $R_{* \text {,disk }}^{\max } / h_{R}$ are small, and $\mathcal{F}_{* \text { max }}^{\text {disk }}$ is relatively insensitive to radius at $R=R_{*, \text { disk }}^{\max }$ due both to the flatness of $V_{\mathrm{c}}$ and $V_{*}^{\text {disk }}$ near this radius. Finally, because there is just a single $\mathcal{F}_{* \text { max }}^{\text {disk }}$ measure per galaxy, we only consider sample errors on this quantity. In this context, all error terms are random with the exception of systematic errors in $H_{0}$ entering through dependence on $\Sigma_{\text {dyn }}$. Typical $\mathcal{F}_{* \text { max }}^{\text {disk }}$ errors for individual galaxies will be $19 \%$ (ran) and 15\% (sys), reducing to $8 \%$ (ran) and $3 \%$ (sys) for averages over 10 galaxies. 


\subsection{Discussion: Baryon fraction, $\mathcal{F}_{\text {bar }}$}

The baryon fraction is given by the ratio of the baryon mass to the total dynamical mass within some radius: $\mathcal{F}_{\text {bar }} \equiv \mathcal{M}_{\text {bar }}^{\text {tot }} / \mathcal{M}_{\text {dyn }}^{\text {tot }}$. The total baryon mass, $\mathcal{M}_{\text {bar }}^{\text {tot }}$, is straightforward to define given our estimate of the stellar disk mass, the atomic and molecular mass, and reasonable estimates for the bulge mass. The latter can be parameterized by the bulge-todisk ratio, e.g., in the $K$-band, with a correction for systematically different mass-to-light ratios as determined by the disk and bulge colors and SPS models calibrated directly by the survey. For galaxies in our survey the dominant term in $\mathcal{M}_{\text {bar }}^{\text {tot }}$ is the stellar disk mass, so in essence the uncertainties in $\mathcal{M}_{\text {bar }}^{\text {tot }}$ are driven by uncertainties in $\Upsilon_{*, \lambda}^{\text {disk }}$.

However, the total mass requires reasonable estimates of the total halo mass, which are difficult to make because of its extended nature and the radial limits of our kinematic data. While H I measurements dramatically improve halo mass estimates, they are still insufficient to constrain the total halo mass with any confidence. This is poignantly illustrated by the analysis in Verheijen (1997) of the Ursa Major galaxy sample. Even ignoring an isothermal halo (which has infinite mass), and considering only a single, mathematical form for the halo density profile which yields a finite and well-defined total mass 9 the estimated fraction of luminous to dark matter $\left(\mathcal{F}_{\text {bar }}\right)$ varies significantly for individual galaxies depending on how the dark-matter profile is constrained. Were we to consider variants of the density profile form, the uncertainty on the total mass for a single galaxy would increase further still.

To bring this point home more clearly, we have examined the logarithmic derivative of changes in the estimated halo-mass $\left(\mathcal{M}_{\text {dyn }}^{\text {halo }}\right)$ with changes in the baryon mass for a given galaxy. We use the Ursa Major sample and fitting done by Verheijen (1997), parameterizing the baryon mass with the $K$-band mass-to-light ratio. Fits to the halo mass and scale were constrained by the observed H I rotation curve, the observed H I mass distribution, and a stellar mass distribution based on the $K$-band luminosity profile and a choice for $\Upsilon_{*, K}$ (constant with radius). Three choices of $\Upsilon_{*, K}$ were used, corresponding to the so-called "maximum disk," "Bottema disk," and an intermediate value. Figure 17 shows the distribution of $\Delta \ln \mathcal{M}_{\text {dyn }}^{\text {halo }} / \Delta \ln \Upsilon_{*, K}$ versus $\Delta \ln \Upsilon_{*, K}$ for both high- and low-surface-brightness subsets of the Ursa Major sample. While there is no clear differences between these subsets, a trend of larger scatter with smaller $\Delta \ln \Upsilon_{*, K}$ is evident. It is reasonable to surmise that there are uncertainties associated with the logarithmic derivative directly related to $\Delta \ln \Upsilon_{*, K}$, i.e., the $(\log )$ size of the interval in $\Upsilon_{*, K}$ used to measure the derivative. The dotted lines in the plot show this error model, which looks plausible. On this basis we calculate

\footnotetext{
${ }^{9}$ Here we consider a Hernquist (1990) profile.
} 
the mean and error in the mean for the appropriately weighted distribution to find:

$$
\Delta \ln \mathcal{M}_{\text {dyn }}^{\text {halo }} / \Delta \ln \Upsilon_{*, K}=1.32 \pm 0.32
$$

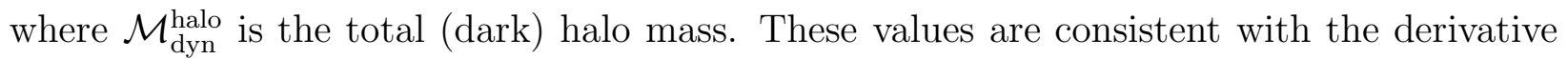
being equal to unity. In other words, $\Upsilon_{*, K}$ and total halo mass $\left(\mathcal{M}_{\text {dyn }}^{\text {halo }}\right)$ are close to 1:1 covariant. What is happening physically with the model is that as $\Upsilon_{*, K}$ increases the halo scale-radius is forced to increase. The mathematical nature of the model is such that $\mathcal{M}_{\text {dyn }}^{\text {halo }}$ also increases with increasing scale-size to produce a 'best fit.' This is not unlike the conspiracy first noted by Bosma (1981) between the mass of the H I disk and that required to make the observed rotation curve flat (explored more recently by, e.g., Hoekstra et al. 2001, and references therein). Here, however, the conspiracy is between the stellar and halo masses, and purely a result of the fitting degeneracy.

This result emphasizes that whatever errors there are in $\Upsilon_{*, K}, \mathcal{F}_{\text {bar }}$ will remain constant in a statistical sense. The nominal value of $\mathcal{F}_{\text {bar }}$, however, will be set by the nature of the rotation curve shape, the halo model, and the way it is fit. All of these differences will lead to a wide range of $\mathcal{F}_{\text {bar }}$ which are dominated by systematic effects. Of course, with a different halo model, we may find a different result, but so too is the value of $\mathcal{F}_{\text {bar }}$ likely to change. The primary point is that $\mathcal{F}_{\text {bar }}$ is conceptually unsatisfactory given what can be observed. While future lensing estimates may make it possible to make a statistical determination of total mass, for the moment a more robust observational quantity is $\mathcal{F}_{* \text { max }}^{\text {disk }}$.

\section{SUMMARY AND CONCLUSIONS}

Our entire error-budget analysis of the DMS precision and accuracy is summarized graphically in Figure 16, illustrating the trends of random and systematic errors on $\Sigma_{\text {dyn }}$, $\Upsilon_{*, K}^{\text {disk }}$, and $\mathcal{F}_{*, \max }^{\text {disk }}$ with disk inclination. We show results adopting the nominal parameters given in the previous section, but differentiate results for kinematic inclinations and inclinations inferred from $K$-band iTF.

The break-down of the errors, adopting kinematic inclinations at $i=30^{\circ}$, are given in Tables 1-3. We have divided the inventory into three groups of random (ran) measurement errors, systematic errors for which we correct (and in this case we are interested in the estimated residual error to this correction, including it in our random error budget), and finally the systematic (sys) errors for which we cannot correct. The latter are an important delineation because they represent systematic errors beyond the control of this and other current astronomical experiments. These systematic errors are comparable to our random errors for individual galaxies, but are five times lower than the random errors when we 
average results over a subset of the survey. The reason for this is simply because many of the systematics for individual galaxies are not systematic for the sample as a whole.

Note that the dominant errors for all quantities of interest are the SVE axial ratio $\alpha$ and inclination (random errors), and the disk oblateness $q$ (systematic errors); they dominate the error on $\Sigma_{\text {dyn }}$, and propagate to dominate the errors on $\Sigma_{*}, \Upsilon_{*}^{\text {disk }}$, and even $\mathcal{F}_{*, \text { max }}^{\text {disk }}$. Random errors in $\alpha$ and inclination would need to be lowered (each) by a factor of 4.5 for other terms to become appreciable (notably $\sigma_{\text {obs }}$ ). Random errors in $\Sigma_{\text {dyn }}$ would have to decreased four-fold for extinction uncertainties to dominate the random errors in $\Upsilon_{*}^{\text {disk }}$ in the $B$ band. Even if our extinction uncertainties are optimistic, they are inconsequential for the error budget for our reddest bands sampling the stellar continuum. For $\mathcal{F}_{* \text {,max }}^{\text {disk }}$, the inclination errors associated with the deprojection of the rotation speed are about 1.7 times lower than the contributions from errors in $\Sigma_{\text {dyn }}$.

Systematic errors in all cases are independent of inclination. Random errors, however, are strongly inclination dependent, and in general increase with inclination. The one exception is the disk fraction $\mathcal{F}_{* \text {,max }}^{\text {disk }}$, which rises steeply at smaller inclinations below $30^{\circ}$ if kinematic inclinations are adopted. In general, adopting iTF inclinations leads to smaller random errors below $i=28^{\circ}$, but the gains are only significant for individual galaxies, except for $\mathcal{F}_{*, \text { max }}^{\text {disk }}$. The reason for the reduced error sensitivity of ensemble $\Sigma_{\text {dyn }}$ and $\Upsilon_{*, K}^{\text {disk }}$ measurements to the choice of inclination estimator is due to the additional errors terms which, for individual galaxies are systematic, but for the ensemble become random, and are independent of inclination. Because of this modest improvement using iTF for galaxy ensemble estimates of $\Sigma_{\text {dyn }}$ and $\Upsilon_{*, K}^{\text {disk }}$, and because $\mathcal{F}_{*, \max }^{\text {disk }}$ is most relevant in the context of knowing independently where a galaxy lies on the TF relation, we prefer kinematic inclinations. This, however, does not preclude the interesting use of iTF for calibrating $\Sigma_{\text {dyn }}$ and $\Upsilon_{*}^{\text {disk }}$ at very low inclination.

Our findings show that typical errors for individual galaxies in our survey will be $28 \%$ (ran) and $26 \%$ (sys) for $\Sigma_{\text {dyn }} ; 32 \%$ (ran) and $30 \%$ (sys) for $\Upsilon_{*}^{\text {disk }}$, weakly dependent on band-pass; and $19 \%$ (ran) and $15 \%$ (sys) for $\mathcal{F}_{*, \max }^{\text {disk }}$. Dividing the sample into quartiles (e.g., 10 galaxies binned by color, luminosity, or surface-brightness) will yield errors of $12 \%$ (ran) and $4 \%$ (sys) for $\Sigma_{\mathrm{dyn}} ; 14 \%$ (ran) and $6 \%$ (sys) for $\Upsilon_{*}^{\text {disk}}$; and $8 \%$ (ran) and $3 \%$ (sys) for $\mathcal{F}_{*, \max }^{\text {disk }}$. These numbers compare favorably to the DMS goal of achieving $30 \%$ errors in $\Upsilon_{*}^{\text {disk }}$. Hence the DiskMass Survey will be able to break the disk-halo degeneracy for individual intermediate-type spiral galaxies in the survey, and calibrate $\Upsilon_{*}^{\text {disk }}$ to sufficient accuracy and precision so that SPS models can be used to break the disk-halo degeneracy in other rotation-curve samples in the nearby universe and at high redshift. This will open the door to measuring the detailed shape of dark-matter halos and understanding the structure and 
formation of galaxies.

Research was supported under NSF/AST-9618849, NSF/AST-997078, NSF/AST-0307417, AST-0607516 (M.A.B.); Spitzer GO-30894 (RAS and MAB); NSF/OISE-0754437 (K.B.W.). M.A.W.V. and T.M. acknowledge the Leids Kerkhoven-Bosscha Fonds for travel support. M.A.B. acknowledges support from the Wisconsin Alumni Research Foundation Vilas Fellowship and the University of Wisconsin College of Letters \& Science Ciriacks Faculty Fellowship.

\section{A. Errors on Inclination}

\section{A.1. Velocity-field Fitting Estimates}

Based on a preliminary analysis of roughly half the Phase- $\mathrm{A} \mathrm{H} \alpha$ sample, we parameterize the errors from inclined-disk model fits to the kinematic data, described in Paper I and Andersen (2001). We find the error distribution is roughly log-linear:

$$
\log d i=a+b \times i
$$

with the slopes and intercepts given in Table A1. These values form a lower envelope (best, or smallest inclination errors at a given inclination); a mid-point to the main grouping of points (mid); and upper envelope to this main grouping (worst); an outside envelope to the very worst cases (extreme); and are rationalized to cross at $82 \mathrm{deg}$ at a value $d i=0.2 \mathrm{deg}$. These values for $d i$ can then be used directly with the formulae below for the logarithmic errors in $\mathcal{M}_{\text {dyn }}^{\text {tot }}$ and $\Sigma_{\text {dyn }}$.

\section{A.2. Inverse Tully-Fisher Estimates}

The Tully-Fisher relation is parameterized as

$$
M_{j}=c_{1, j}+c_{2, j} \log \left(W_{R} / \sin i\right)
$$

where $M_{j}$ is the absolute magnitude in the $j^{\text {th }}$ band, $W_{R}$ is the line-width, equivalent roughly to $2 V_{\text {obs }}$, and $c_{2, j}$ is the TF slope. With inversion and differentiation, the inclination error in radians is given by

$$
\frac{d i}{\tan i}=\sqrt{\left(\Delta \ln V_{\text {obs }}\right)^{2}+\left(\frac{\ln 10}{c_{2, j}} d M_{j}\right)^{2}}
$$


where $d M_{j}$ is the scatter in the TF relationship (magnitudes). This quantity is constant with inclination. Expression for the total mass and disk-mass error come from inserting equation A1 into equations B1 and B11 respectively.

For the data in this survey, the dominant contributor to the iTF inclination error comes from the scatter in the TF relation itself. The example in Figure 16 in Paper I is typical of the $\mathrm{H} \alpha$ data quality, but extreme in the sense that the galaxy is very nearly face-one, i.e., under $3.5 \mathrm{deg}$ inclination assuming an intrinsic rotation velocity of $200 \mathrm{~km} \mathrm{~s}^{-1}$ or greater. The velocity error is about $2 \%$, a little less than the contribution from 0.1 mag scatter and a -9 slope for the TF relation. In general, galaxies in our sample are at higher inclination and the TF scatter dominates equation B11. In this case, for large ellipsoid errors (e.g., $50 \%$ ), differences in $\Delta \ln \Sigma_{\text {dyn }}$ are dominated by the SVE shape; TF slope and scatter are almost inconsequential in the range 0.1 and $0.5 \mathrm{mag}$ and slopes between -5 and -9 , typical of what is observed in the optical through near-infrared bands. For smaller ellipsoid errors (e.g., 10\%), TF scatter dominates the variation in $\Delta \ln \Sigma_{\text {dyn }}$ with $i$ for $\Delta \ln \Sigma_{\text {dyn }}>0.1$ (or $i>30 \mathrm{deg})$. At smaller values, TF scatter and SVE shape lead to comparable variations in $\Delta \ln \Sigma_{\text {dyn }}$ at a given $i$. TF slope has a much smaller effect.

\section{B. Error Formulae}

This Appendix serves three agendas: (1) to provide analytic expressions for random (indicated as $[\mathrm{ran}]$ ) and systematic (indicated as $[$ sys]) errors of key survey quantities for individual survey galaxies; (2) in so doing to identify which terms contribute to these two types of errors, and how this changes when considering results for individual survey galaxies versus the survey as a whole; and (3) to isolate the contribution from uncertainties in disk inclination and the SVE. The latter serves to focus on quantities central to the rationale behind a near face-on strategy for the DMS. Error-functions are given as logarithmic derivatives for disk-mass surface density $\left(\Sigma_{\mathrm{dyn}}\right)$, the disk stellar mass-to-light ratio $\left(\Upsilon_{*}^{\text {disk }}\right)$, the disk mass-fraction $\left(\mathcal{F}_{*, \text { max }}^{\text {disk }}\right)$, and total mass $\left(\mathcal{M}_{\text {dyn }}^{\text {tot }}\right)$.

\section{B.1. Errors on $\Sigma_{\mathrm{dyn}}$}

$\Sigma_{\text {dyn }}$ (equation 9) is estimated via line-of-sight velocity dispersion, corrected for instrumental effects and SVE projection (equation 6), estimated disk oblateness (equation 1), apparent disk scale-length, and distance. Deprojection requires information on inclination. With distinctions made in $\S 5$ and $\S 5.1$, the logarithmic derivative of $\left\langle\Sigma_{\text {dyn }}\right\rangle$ for disk-averaged 
measurements of an individual galaxy can be separated into a random term,

$$
\begin{aligned}
& \left(\Delta \ln \left\langle\Sigma_{\text {dyn }}\right\rangle\right)^{2}[\text { ran }]=0.4\left(\Delta \ln h_{R}\right)^{2}+ \\
& \frac{4}{N_{\text {disk }}}\left\{\left[\left(\frac{\sigma_{\text {obs }}}{\sigma_{\text {LOS }}}\right)^{2} \Delta \ln \sigma_{\text {obs }}\right]^{2}+\left[\left(\frac{\sigma_{\text {beam }}}{\sigma_{\text {LOS }}}\right)^{2} \Delta \ln \sigma_{\text {beam }}\right]^{2}+\right. \\
& \left.\quad\left[\left(\frac{\sigma_{\text {inst }}}{\sigma_{\text {LOS }}}\right)^{2} \Delta \ln \sigma_{\text {inst }}\right]^{2}+\left(\Delta \ln \sigma_{\text {tpl }}\right)^{2}\right\}+ \\
& \frac{\tan ^{4} i^{2}}{\alpha^{4} \gamma}\left(\left\{\left[2 \alpha^{2} \gamma+\left(1+\beta^{2}\right) \sec ^{2} i\right] \frac{d i}{\tan i}\right\}^{2}+\left[\left(1+\beta^{2}\right) \Delta \ln \alpha\right]^{2}+\left(\beta^{2} \Delta \ln \beta\right)^{2}\right)
\end{aligned}
$$

and a systematic term,

$$
\left(\Delta \ln \left\langle\Sigma_{\text {dyn }}\right\rangle\right)^{2}[\text { sys }]=(\Delta \ln k)^{2}+(\Delta \ln q)^{2}+0.4\left[\left(\Delta \ln D_{\mathrm{H} 0}\right)^{2}+\left(\Delta \ln D_{\text {flow }}\right)^{2}\right],
$$

where for the simplicity of notation, the quantities $\gamma, \sigma_{\mathrm{LOS}}, \sigma_{\mathrm{obs}}, \sigma_{\text {beam }}, \sigma_{\text {inst }}$, and $\sigma_{\mathrm{tpl}}$ are all averages over azimuth and radius (e.g., $\gamma$ in equation B1 represents $\langle\bar{\gamma}\rangle$, where $\bar{\gamma}$ is the azimuthally-averaged value defined by equation 5). Here, $d i$ is expressed in radians, and the logarithmic derivatives of individual terms are characteristic values for an azimuthal ring at a single radius. The errors on $\Sigma_{\text {dyn }}$ at a single radius for an individual galaxy only differ by

the removal of the $N_{\text {disk }}^{-1}$ factor in the middle set of terms in equation B1; $N_{\text {disk }}^{-1}$ specifies the number of radial rings of averaged (stacked) fibers. These terms have different dependence on $\sigma_{\mathrm{LOS}}$ because of the way they enter into the calculation of $\sigma_{\mathrm{LOS}}$. Suitable values for each of the above terms are itemized in Table 1. Survey systematic errors for $\Sigma_{\text {dyn }}$ include only uncertainty distances due to $\mathrm{H}_{0}$.

Error-dependence on inclination and SVE include only the last set of terms in equation B1, which follow from setting $\Delta \ln \left\langle\Sigma_{\text {dyn }}\right\rangle=\Delta \ln \left(\bar{\gamma} \cos ^{2} i\right)$. Neither $\gamma \cos ^{2} i$ or $\bar{\gamma} \cos ^{2} i$ are rapidly varying functions of $i ; \bar{\gamma} \cos ^{2} i$ is unity for all $i$ for $\alpha=\beta=1$, varies by less than a factor of 2 for $\alpha=\beta=0.7$, and by less than a factor of 4 for $\alpha=\beta=0.4$. This fact, strangely at odds with the geometric projection of $\sigma_{z}$, arises because we are relying on the SVE shape to derive $\sigma_{z}$ from the line-of-sight measurement. In contrast, the geometric projection of $\sigma_{z}$ can be written logarithmically as $\sigma_{z} \cos i / \sigma_{\mathrm{LOS}}=1 / \sqrt{\bar{\gamma}}$. The systematic error on deriving $\sigma_{z}$ from $\sigma_{\mathrm{LOS}}$ (expressed as a fraction) then must be proportional to $\sigma_{\mathrm{LOS}} / \sigma_{z} \cos i-1=\sqrt{\bar{\gamma}}-1$.

\section{B.2. Errors on $\Upsilon_{*, \lambda}^{\text {disk }}$}

$\Upsilon_{*, \lambda}^{\text {disk }}$ (equation 10, $§ 5.2$ ) is estimated via the stellar disk surface-density and the extinctioncorrected flux density. The former is a function of $\Sigma_{\mathrm{dyn}}, \Sigma_{\mathrm{HI}}$, and a parameterization of the 
molecular gas component. The logarithmic derivative of $\left\langle\Upsilon_{*, \lambda}^{\text {disk }}\right\rangle$ can be written as

$$
\left(\Delta \ln \left\langle\Upsilon_{*, \lambda}^{\text {disk }}\right\rangle\right)^{2}=\left(\Delta \ln \left\langle I_{\lambda}^{c}\right\rangle\right)^{2}+\left(\Delta \ln \left\langle\Sigma_{*}\right\rangle\right)^{2} .
$$

The expression for the first term is

$$
\left(\Delta \ln \left\langle I_{\lambda}^{c}\right\rangle\right)^{2}=\frac{0.85}{N_{\text {disk }}}\left[\left\langle\Delta \mu_{\lambda}\right\rangle^{2}+0.85\left\langle\Delta \mathrm{A}_{\lambda}\right\rangle^{2}\right]
$$

where $\left\langle\Delta \mu_{\lambda}\right\rangle$ and $\left\langle\Delta \mathrm{A}_{\lambda}\right\rangle$ are the characteristic errors in a single ring for the surface-brightness (mag $\operatorname{arcsec}^{-2}$ ) and extinction correction (mag), respectively. This is a suitable approximation for magnitude errors below 1. The second term can be written as:

$$
\begin{aligned}
&\left(\Delta \ln \left\langle\Sigma_{*}\right\rangle\right)^{2}=\left(\frac{\xi \Delta \ln \xi}{1-\xi}\right)^{2}+ \\
&(1-\xi)^{2}\left[\left(\frac{\Sigma_{\mathrm{dyn}}}{\Sigma_{*}} \Delta \ln \left\langle\Sigma_{\mathrm{dyn}}\right\rangle\right)^{2}+\left(\frac{1.4 \Sigma_{\mathrm{HI}}}{\sqrt{N_{\mathrm{disk}}} \Sigma_{*}}\left\langle\Delta \ln \Sigma_{\mathrm{HI}}\right\rangle\right)^{2}\right]
\end{aligned}
$$

and

$$
(\Delta \ln \xi)^{2}=(\Delta \ln \eta)^{2}+\left(\frac{\xi}{1.4 \eta} \frac{\mathcal{M}_{\mathrm{dyn}}^{\text {disk }}}{\mathcal{M}_{\mathrm{HI}}}\right)^{2}\left[\left(\Delta \ln \mathcal{M}_{\mathrm{dyn}}^{\text {disk }}\right)^{2}+\left(\Delta \ln \mathcal{M}_{\mathrm{HI}}\right)^{2}\right]
$$

where $\mathcal{M}_{\mathrm{HI}}, \mathcal{M}_{*}^{\text {disk }}$, and $\mathcal{M}_{\text {dyn }}^{\text {disk }}$ are the H I, stellar, and total disk-mass components. Approximating $\Delta \ln \mathcal{M}_{i}=N_{i}^{-1 / 2}\left\langle\Delta \ln \Sigma_{i}\right\rangle=\Delta \ln \left\langle\Sigma_{i}\right\rangle$ for the $i^{\text {th }}$ mass-component measured in $N_{i}$ radial rings, where $\left\langle\Delta \ln \Sigma_{i}\right\rangle$ is the characteristic error in $\Sigma_{i}$ for a single ring, and $\Delta \ln \left\langle\Sigma_{i}\right\rangle$ is the error in the mean value, we then collect random and systematic error terms to obtain:

$$
\begin{aligned}
\left(\Delta \ln \left\langle\Sigma_{*}\right\rangle\right)^{2}[\text { ran }]= & {\left[\frac{1}{N_{\text {disk }}}\left(1.4(1-\xi) \frac{\Sigma_{\mathrm{HI}}}{\Sigma_{*}}\right)^{2}+\frac{1}{N_{\text {atom }}}\left(\frac{\xi^{2}}{1.4 \eta(1-\xi)} \frac{\mathcal{M}_{\mathrm{dyn}}^{\text {disk }}}{\mathcal{M}_{\mathrm{HI}}}\right)^{2}\right]\left\langle\Delta \ln \Sigma_{\mathrm{HI}}\right\rangle^{2}+} \\
& {\left[\left((1-\xi) \frac{\Sigma_{\mathrm{dyn}}}{\Sigma_{*}}\right)^{2}+\left(\frac{\xi^{2}}{1.4 \eta(1-\xi)} \frac{\mathcal{M}_{\mathrm{dyn}}^{\text {disk }}}{\mathcal{M}_{\mathrm{HI}}}\right)^{2}\right]\left(\Delta \ln \left\langle\Sigma_{\mathrm{dyn}}\right\rangle\right)^{2}[\text { ran }] } \\
\left(\Delta \ln \left\langle\Sigma_{*}\right\rangle\right)^{2}[\text { sys }]= & \left(\frac{\xi}{1-\xi}\right)^{2}(\Delta \ln \eta)^{2}+ \\
& {\left[\left((1-\xi) \frac{\Sigma_{\mathrm{dyn}}}{\Sigma_{*}}\right)^{2}+\left(\frac{\xi^{2}}{1.4 \eta(1-\xi)} \frac{\mathcal{M}_{\mathrm{dyn}}^{\text {disk }}}{\mathcal{M}_{\mathrm{HI}}}\right)^{2}\right]\left(\Delta \ln \left\langle\Sigma_{\mathrm{dyn}}\right\rangle\right)^{2}[\text { sys }] \quad(\mathrm{B} 8) }
\end{aligned}
$$

We relate all ratios involving mass or surface-density to $\mathcal{M}_{*}^{\text {disk }} / \mathcal{M}_{\mathrm{HI}}$ with the identities of $\mathcal{M}_{\text {dyn }}^{\text {disk }} / \mathcal{M}_{\mathrm{HI}}=\mathcal{M}_{*}^{\text {disk }} / \mathcal{M}_{\mathrm{HI}}+1.4(1+\eta), \Sigma_{\mathrm{dyn}} / \Sigma_{*}=(1-\xi)^{-1}+1.4\left(\Sigma_{\mathrm{HI}} / \Sigma_{*}\right)$, and $\Sigma_{\mathrm{HI}} / \Sigma_{*} \sim$ 
$\left(\mathcal{M}_{\mathrm{HI}} R_{*}^{2} / \mathcal{M}_{*}^{\text {disk }} R_{\mathrm{HI}}^{2}\right)$, where $R_{*}$ and $R_{\mathrm{HI}}$ are the characteristic length-scales of the stellar and $\mathrm{H}$ I distribution. Suitable values for each of the above terms are discussed in $\S 5.2$, and itemized in Table 2.

Random errors in $\Upsilon_{*, \lambda}^{\text {disk }}$ include the sum of equations (B4) and (B7); systematic errors are equal to equation (B8). Survey systematic errors and inclination dependence for $\Upsilon_{*, \lambda}^{\text {disk }}$, come from terms in equation (B8) with $\Sigma_{\mathrm{dyn}}[s y s]$, as noted respectively in the previous section for equation (B2).

\section{B.3. Errors on $\mathcal{F}_{* \text { max }}^{\text {disk }}$ and $\mathcal{M}_{\text {dyn }}^{\text {tot }}$}

$\mathcal{F}_{*, \text { max }}^{\text {disk }}$ is estimated via the mass of the stellar disk, it's oblateness, and the projected total circular rotation speed $\left(V_{\text {obs }}-V_{\text {sys }}\right.$; see $\left.\S 5.3\right)$, corrected for inclination. Using the results in the previous section we find:

$$
\begin{gathered}
\left(\Delta \ln \mathcal{F}_{*, \text { max }}^{\text {disk }}\right)^{2}[\text { ran }]=\frac{1}{4}\left(\Delta \ln \left\langle\Sigma_{*}\right\rangle\right)^{2}[\text { ran }]+\left(\Delta \ln V_{\text {obs }}\right)^{2}+(\text { di } / \tan i)^{2} \\
\left(\Delta \ln \mathcal{F}_{*, \text { max }}^{\text {disk }}\right)^{2}[\text { sys }]=\frac{1}{4}\left[\left(\Delta \ln f_{*}^{\text {disk }}\right)^{2}+\left(\Delta \ln \left\langle\Sigma_{*}\right\rangle\right)^{2}[\text { sys }]\right]
\end{gathered}
$$

We subsume the errors in the kinematic center and position angle of a galaxy in the errors for $V_{\text {obs }}$ and inclination, and ignore the negligible errors on $V_{\text {sys }}$. The last term in (B9) is roughly $2 d i / i$ for small $i$, where $d i$ is expressed in units of radians. The only systematic errors in this quantity for the survey ensemble stem from distance, coupled to $\Sigma_{*}$ via $\Sigma_{\text {dyn }}$ $(\mathrm{B} 2, \mathrm{~B} 8)$. Inclination-dependence to the random errors are from the last term in equation B9 and terms in B1 related to $\Sigma_{*}$.

Errors in the total potential mass at a given radius are:

$$
\begin{gathered}
\left.\left(\Delta \ln \mathcal{M}_{\mathrm{dyn}}^{\mathrm{tot}}\right)^{2}[\text { ran }]=4\left(\Delta \ln V_{\mathrm{obs}}\right)^{2}+(d i / \tan i)^{2}\right) \\
\left(\Delta \ln \mathcal{M}_{\mathrm{dyn}}^{\mathrm{tot}}\right)^{2}[\text { sys }]=\left(\Delta \ln f_{\mathrm{tot}}\right)^{2}
\end{gathered}
$$

where $f_{\text {tot }}$ is a measure of the flattening of the potential. Inclination-dependence only arises from the deprojections of the observed tangential velocity $V_{\text {obs }}$.

\section{REFERENCES}

Akritas, M. G., Bershady, M. A. 1996, ApJ, 470, 706

Aoki, T. E., Hiromoto, N., Takami, H., Okamura, S. 1991, PASJ, 43, 755 
Andersen, D. R. 2001, Ph.D. Thesis, Penn State University

Andersen, D. R., \& Bershady, M. A. 2003, ApJ, 599, L79

Andersen, D. R., Bershady, M. A., Sparke, L. S., Gallagher, J. S., Wilcots, E. M., van Driel, W., Monnier-Ragaigne, D. 2006, ApJS, 166,505

Andersen, D. R., Walcher, C. J., Boker, T., Ho, Luis C., van der Marel, R. P., Rix, H.-W., Shields, J. C. 2008, ApJ, 688, 990

Bahcall, J, \& Casertano, S. 1984, ApJ, 284, L35

Barth, A., Ho, L. C., Sargent, W. L. W. 2002, AJ, 124, 2607

Begeman, K. G. 1989, A\&A, 223, 47

Bender, R., Saglia, R. P., Gerhard, O. E. 1994, MNRAS, 269, 785

Bershady, M. A., Andersen, D. R., Verheijen, M. A. W., Westfall, K. M., Crawford, S. M., Swaters, R. A. 2005, ApJS, 156, 311

Bershady, M. A., Verheijen, M. A. W., Swaters, R. A., Andersen, D. R., Westfall, K. M., Martinsson, T. 2009, ApJ, submitted (Paper I)

Binney, J., \& Merrifield, M. 1998, "Galactic Astronomy," Princeton University Press

Binney, J., \& Tremaine, S. 1987, "Galaxy Dynamics", Princeton University Press

Bizyaev, D. \& Mitronova, S. 2002, A\&A, 389, 795

Bizyaev, D. \& Mitronova, S. 2009, ApJ, 702, 1567

Boissier, S., Boselli, A., Buat, V., Donas, J., Milliard, B. 2004, A\&A, 424, 465

Bosma, A. 1981, AJ, 86, 1825

Bottema, R. 1993, A\&A, 275, 16

Bottema, R. 1997, A\&A, 328, 517

Calzetti, D., Kinney, A., L., \& Storchi-Bergmann, T. 1994, ApJ, 429, 582

Casertano, S. 1983, MNRAS, 203, 735

Casoli, E., et al. 1998, A\&A, 331, 451

Coluzzi, R. 1993, Bull. Inf. Centre Donnees Stellaires, 43, 7

Courteau, S. 1997, AJ, 114, 2402

Dalcanton, J. J., Yoachim, P. \& Bernstein, R. A. 2004, ApJ, 608, 189

de Grijs, R., van der Kruit, P. C. 1997, A\&AS, 327, 966

de Grijs, R. 1998, MNRAS, 299, 595 
de Jong, R. 1996a, A\&A, 313, 377

de Jong, R. 1996b, A\&AS, 118, 557

de Bruyne, V., De Rijcke, S., Dejonghe, H., Zeilinger, W. W. 2004, MNRAS, 349, 461

Domingue, D. L., Keel, W. C., White, R. E. 2000, ApJ, 545, 171

Falcón-Barroso, J. et al. 2006, MNRAS, 369, 529

Fisher, D. 1997, AJ, 113, 950

Fisher, D. 1997, AJ, 113, 950

Franx, M. \& Illingworth, G. 1988, ApJ, 327, L55

Freeman, K. C. 1970, ApJ, 160, 811

Fry, A. M., Morrison, H. L., Harding, P., Boroson, T. A. 1999, AJ, 118, 1209

Fukugita, M., Ichikawa, T., Gunn, J. E., Doi, M., Shimasaky, K., Schneider, D. P. 1996, AJ, 111,1748

Gerhard, O., Jeske, G., Saglia, R. P., Bender, R. 1998, MNRAS, 295, 197

Herrmann, K. A., and Ciardullo, R. 2009, ApJ, accepted (arXiv:0910.0266v1)

Hernquist, L. 1990, ApJ, 356, 359

Hoekstra, H., van Albada, T. S., Sancisi, R. 2001, MNRAS, 323, 453

Holwerda, B. W., Gonzalez, R. A., Allen, R. J., van der Kruit, P. C. 2005, AJ, 129, 1396

Howk, J. C., \& Savage, B. D. 1999, AJ, 117, 2077

Kamphuis, P., Holwerda, B. W., Allen, R. J., Peletier, \& van der Kruit, P. C. 2007, A\&A, 471, L1

reference Keel, W. C., \& White, R. E. 2001, AJ, 121, 1442

Kregel, M., van der Kruit, P. C., De Grijs, R. 2002, MNRAS, 334, 646

Kregel, M., van der Kruit, P. C., Freeman, K. C. 2004, MNRAS, 351, 1247

Kregel, M., van der Kruit, P. C., Freeman, K. C. 2005, MNRAS, 3581, 503

Le Borgne, D., Rocca-Volmerange, B., Prugniel, P., Lançon, A., Fioc, M., and Soubiran, C. 2004, A\&A, 425, 881

Leroy, A. K., Walter, F., Brinks, E., Bigiel, F., de Blok, W. J. G., Madore, B., Thornley, M. D. 2008, AJ, 136, 2782

MacArthur, L, Courteau, S., Holtzman, J. 2003, ApJ, 582, 689

Misiriotis, A., Kylafis, N. D., Papamastorakis, J., Xilouris, E. M. 2000, A\&A, 353, 117 
Misiriotis, A., Popescu, C. C., Tuffs, R., Kylafis, N. D. 2001, A\&A, 372, 775

Mould, J. et al. 2000, ApJ, 529, 786

Morrison, H. L., Boroson, T. A., Harding, P. 1994, AJ, 108, 1191

Nelson, C. H., Whittle, M. 1995, ApJS, 99, 67

Obreschkow, D., \& Rawlings, S. 2009, MNRAS, 394, 1857

Olling, R. P. 1996, AJ, 112

Pohlen, M., Dettmar, R.-J., Lutticke, R., and Schwarzkopf, U. 2000, A\&AS, 144, 405

Popescu, C. C., Misiriotis, A., Kylafis, N. D., Tuffs, R. J., Fischera, J. 2000, A\&A, 362, 138

Regan, M. W., et al. 2001, ApJ, 561, 218

Rix, H.-W., White, S. D. M. 1992, MNRAS, 254, 389

Schwarzkopf, U., Dettmar, R.-J. 2000, A\&A, 361, 451

Seth, A. C., Dalcanton, J. J., and De Jong, R. S. 2005, AJ, 130, 1574

Shapiro, K., Gerssen, J., van der Marel, R.P. 2003, AJ, 126, 2707

Simkin, S. 1974, A\&A, 31, 129

Statler, T. S., 1995, AJ, 109, 1371

Swaters, R. A., Verheijen, M. A. W., Bershady, M. A., Andersen, D. R. 2003, ApJ, 587, L19

Tamura, K., Jansen, R. A., \& Windhorst, R. A. 2009, AJ, 138, 1634

Tully, R. B., \& Fisher, J. R. 1977, A\&A, 54, 661

van Albada, T. S., Bahcall, J. N., Begeman, K., Sancisi, R. 1985, ApJ, 295, 305

van den Bergh, S., 1960, ApJ, 131, 215

van der Kruit, P. C., \& Searle, L. 1981, A\&A, 95, 105

van der Kruit, P. C., \& Freeman, K. C. 1984, ApJ, 278, 81

van der Kruit, P. C., \& Freeman, K. C. 1986, ApJ, 303, 556

van der Kruit, P. C. 1988, A\&A, 192, 117

van der Kruit, P. C., \& de Grijs, R. 1999, A\&A, 352, 129

van der Marel, R. P., \& Franx, M. 1993, 407, 525

Verheijen, M. A. W. 1997, Ph.D. thesis, University of Groningen

Verheijen, M. A. W. 2001, ApJ, 563, 694

Verheijen, M. A. W., \& Sancisi, R. 2001, A\&A, 370, 765 
Wainscoat, R. J, Freeman, K. C., Hyland, A.R. 1989, ApJ, 337, 163

Westfall, K. B. 2009, Ph.D. Thesis, University of Wisconsin

Xilouris, E. M., Kylafis, N. D., Papamastorakis, J., Paleologou, E. G., Haerendel, G. 1997, A\&A, 325, 135

Xilouris, E. M., Alton, P. B., Davies, J. I., Kylafis, N. D., Papamastorakis, J., Trewhella, M. 1998, A\&A, 331, 894

Xilouris, E. M., Byun, Y. I., Kylafis, N. D., Papamastorakis 1999, A\&A, 344, 868

Yoachim, P. \& Dalcanton, J. J. 2006, AJ, 131, 226

Young, J. D., Knezek, P. M. 1989, ApJ, 347, L55

This preprint was prepared with the AAS LATEX macros v5.2. 
Table 1. $\Sigma_{\mathrm{dyn}}$ Error Budget for Individual Galaxies at $i=30^{\circ}$

\begin{tabular}{|c|c|c|c|c|c|}
\hline \multirow[t]{2}{*}{ Quantity } & \multirow[t]{2}{*}{ Type } & \multirow[t]{2}{*}{ Section } & \multirow[t]{2}{*}{ Equation } & \multicolumn{2}{|c|}{ Log Error } \\
\hline & & & & Quantity & $\Sigma_{\text {dyn }}$ \\
\hline \multicolumn{6}{|c|}{ random } \\
\hline 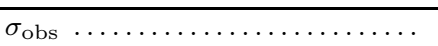 & measurement & 3.3 & $\mathrm{~B} 1$ & 0.030 & 0.027 \\
\hline template mismatch ..... & measured correction & 3.4 & B1 & 0.040 & 0.036 \\
\hline instrumental resolution...$\ldots$. & measured correction & 3.5 .1 & B1 & 0.030 & 0.013 \\
\hline beam smearing $\ldots \ldots \ldots \ldots \ldots$ & measured correction & 3.5 .2 & B1 & 0.500 & 0.001 \\
\hline SVE deprojection, $\alpha \ldots \ldots \ldots$ & measured correction & 3.5 .4 & B1 & 0.250 & 0.204 \\
\hline SVE deprojection, $\beta \ldots \ldots \ldots \ldots$ & measured correction & 3.5 .4 & B1 & 0.050 & 0.013 \\
\hline SVE deprojection, $i \ldots \ldots \ldots$ & measured correction & 3.5 .4 & B1 & 0.116 & 0.184 \\
\hline total SVE deprojection, $\sqrt{\bar{\gamma}} \cos i$ & measured correction & 3.5 .3 & B1 & 0.138 & 0.275 \\
\hline$h_{R} \ldots \ldots \ldots \ldots \ldots \ldots \ldots \ldots$ & measurement & 2.2 & B1 & 0.030 & 0.019 \\
\hline$\Sigma_{\text {dyn }} \ldots \ldots \ldots \ldots \ldots \ldots \ldots$ & total random error & 5.1 & B1 & $\cdots$ & 0.280 \\
\hline \multicolumn{6}{|c|}{ systematic } \\
\hline$h_{R}: h_{z}$ conversion, $q_{R} \ldots \ldots \ldots$ & estimated correction & 2.2 & $\mathrm{~B} 2$ & 0.250 & 0.250 \\
\hline distance, $D_{\text {flow }} \ldots \ldots \ldots \ldots$ & uncorrected systematic & 4 & $\mathrm{~B} 2$ & 0.080 & 0.051 \\
\hline distance, $H_{0} \quad \ldots \ldots \ldots \ldots \ldots$ & uncorrected systematic & 4 & $\mathrm{~B} 2$ & 0.070 & 0.044 \\
\hline vertical distribution, $k \ldots \ldots \ldots$ & uncorrected systematic & 5.1 & $\mathrm{~B} 2$ & $(0.140)$ & $(0.140)$ \\
\hline$\Sigma_{\text {dyn }} \ldots \ldots \ldots \ldots \ldots \ldots \ldots$ & total systematic error & 5.1 & $\mathrm{~B} 2$ & $\ldots$ & 0.259 \\
\hline
\end{tabular}

Note. -()$=$ excluded from final tally. 
Table 2. $\quad \Sigma_{*}$ and $\Upsilon_{*}^{\text {disk }}$ Error Budget for Individual Galaxies at $i=30^{\circ}$

\begin{tabular}{|c|c|c|c|c|c|}
\hline \multirow[t]{2}{*}{ Quantity } & \multirow[t]{2}{*}{ Type } & \multirow[t]{2}{*}{ Section } & \multirow[t]{2}{*}{ Equation } & \multicolumn{2}{|c|}{ Log Error } \\
\hline & & & & Quantity & $\Sigma_{*}, \Upsilon_{*}^{\text {disk }}$ \\
\hline \multicolumn{6}{|c|}{ random } \\
\hline$\ldots \ldots \ldots \ldots \ldots \ldots \ldots$ & measurement & 5.2 & B7 & 0.030 & 0.002 \\
\hline 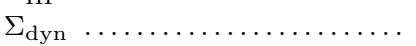 & corrected measurement & 5.2 & B7 & 0.280 & 0.321 \\
\hline$\Sigma_{*} \ldots \ldots \ldots \ldots \ldots \ldots \ldots \ldots \ldots \ldots$ & total random error & 5.2 & B7 & $\ldots$ & 0.321 \\
\hline$\mu_{\lambda} \ldots \ldots \ldots \ldots \ldots \ldots \ldots \ldots$ & measurement & 5.2 & B4 & 0.050 & 0.022 \\
\hline internal extinction, $A_{B} \ldots \ldots$ & estimated correction & 5.2 & B4 & 0.180 & 0.074 \\
\hline internal extinction, $\mathrm{A}_{I} \ldots \ldots \ldots$ & estimated correction & 5.2 & B4 & 0.060 & 0.025 \\
\hline internal extinction, $\mathrm{A}_{K} \ldots \ldots$ & estimated correction & 5.2 & $\mathrm{~B} 4$ & 0.008 & 0.003 \\
\hline$\Upsilon_{*, B}^{\text {disk }} \ldots \ldots \ldots \ldots \ldots \ldots \ldots$ & total random error & 5.2 & B3 & $\ldots$ & 0.331 \\
\hline$\Upsilon_{*, I}^{\text {disk }}$ & total random error & 5.2 & B3 & $\cdots$ & 0.323 \\
\hline$\Upsilon_{*, K}^{\text {disk }} \ldots \ldots \ldots \ldots \ldots \ldots \ldots \ldots$ & total random error & 5.2 & B3 & $\cdots$ & 0.322 \\
\hline \multicolumn{6}{|c|}{ systematic } \\
\hline molecular:atomic mass ratio, $\eta$ & estimated correction & 5.2 & B8 & 0.400 & 0.040 \\
\hline$\Sigma_{\text {dyn }} \ldots \ldots \ldots \ldots \ldots \ldots \ldots \ldots$ & uncorrected measurement & 5.2 & B8 & 0.259 & 0.298 \\
\hline$\Sigma_{*}$ and $\Upsilon_{*}^{\text {disk }}$ & total systematic error & 5.2 & B8,B3 & $\ldots$ & 0.300 \\
\hline
\end{tabular}


Table 3. $\mathcal{F}_{*, \max }^{\text {disk }}$ Error Budget for Individual Galaxies at $i=30^{\circ}$

\begin{tabular}{|c|c|c|c|c|c|}
\hline \multirow[t]{2}{*}{ Quantity } & \multirow[t]{2}{*}{ Type } & \multirow[t]{2}{*}{ Section } & \multirow[t]{2}{*}{ Equation } & \multicolumn{2}{|c|}{ Log Error } \\
\hline & & & & Quantity & $\mathcal{F}_{*, \text { max }}^{\text {disk }}$ \\
\hline \multicolumn{6}{|c|}{ random } \\
\hline$\Sigma_{*} \ldots \ldots, \ldots, \ldots, \ldots$ & corrected measurement & 5.2 & B9 & 0.321 & 0.161 \\
\hline$V_{\text {obs }} \ldots \ldots \ldots \ldots \ldots$ & measurement & 5.3 & B9 & 0.020 & 0.020 \\
\hline velocity deprojection, $i$ & measured correction & 5.3 & B9 & 0.116 & 0.105 \\
\hline $\mathcal{F}_{*, \max }^{\text {disk }} \ldots \ldots \ldots \ldots \ldots$ & total random error & 5.3 & B9 & $\ldots$ & 0.193 \\
\hline \multicolumn{6}{|c|}{ systematic } \\
\hline$\Sigma_{*} \ldots \ldots \ldots \ldots \ldots \ldots$ & uncorrected measurement & 5.2 & B10 & 0.300 & 0.150 \\
\hline disk oblateness, $f_{*}^{\text {disk }}$. & estimated correction & 5.3 & B10 & 0.019 & 0.009 \\
\hline $\mathcal{F}_{*, \max }^{\text {disk }} \ldots \ldots \ldots \ldots$ & total systematic error & 5.3 & $\mathrm{~B} 10$ & $\ldots$ & 0.150 \\
\hline
\end{tabular}


Table A1. Kinematic-Inclination Error Coefficients

\begin{tabular}{lcc}
\hline \hline & $\mathrm{a}$ & $\mathrm{b}$ \\
\hline best & 0.84 & -0.019 \\
mid & 1.26 & -0.024 \\
worst & 1.74 & -0.030 \\
extreme & 2.49 & -0.039 \\
\hline
\end{tabular}



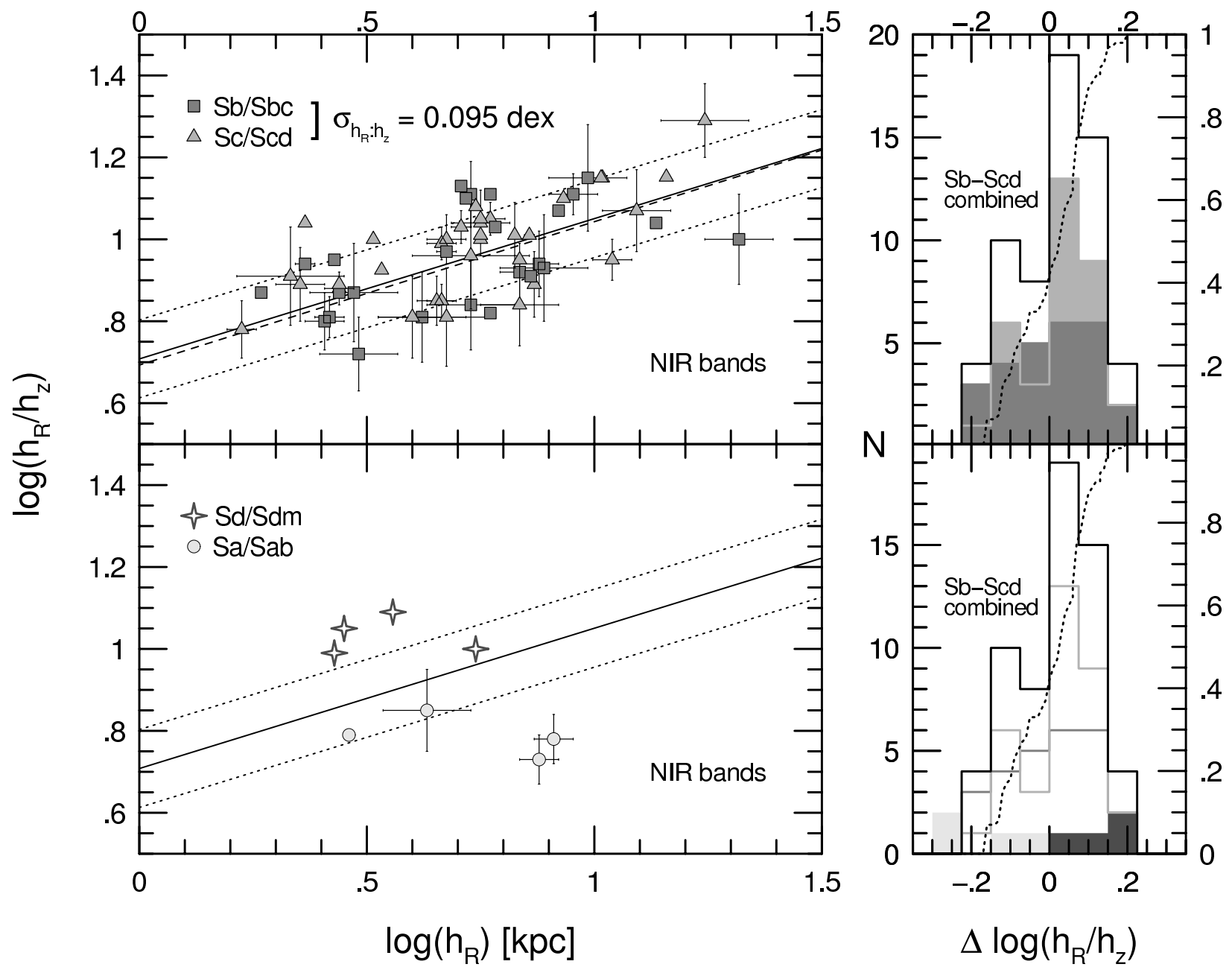

Fig. 1.- Radial to vertical scale-length ratio $\left(h_{R} / h_{z}\right)$ distribution in the $I$ or near-infrared bands for edge-on spiral galaxies. Top panels show intermediate types while bottom panels show early and late types based on a compilation described in the text. Left panels show the distribution versus radial scale length $\left(h_{R}\right)$. The solid line represents a linear leastsquares fit (no rejection) to the Kregel et al. sample for Sb-Scd Hubble-types, typical of the DMS. Dotted lines are the $1 \sigma$ dispersion about these fits, corresponding to 0.095 dex or $25 \%$ random uncertainty in $h_{z}$ per galaxy based on measured radial scale-lengths. The dashed line is a weighted regression with intrinsic scatter (Akritas \& Bershady 1996) to the same subset, yielding comparable results in slope and dispersion. Right panels are histograms of deviations for all galaxies about the best-fit to the Kregel et al. sample, broken down by type. Black solid and dotted lines represent differential and normalized cumlative distributions for the 60 galaxies typed between $\mathrm{Sb}$ and Scd. Intermediate light- and dark-gray histograms (top) and extreme light- and dark-gray historgram (bottom) are the differential distributions for $\mathrm{Sc} / \mathrm{Scd}, \mathrm{Sb} / \mathrm{Sbc}, \mathrm{Sd} / \mathrm{Sdm}$, and $\mathrm{Sa} / \mathrm{Sab}$ types respectively. 

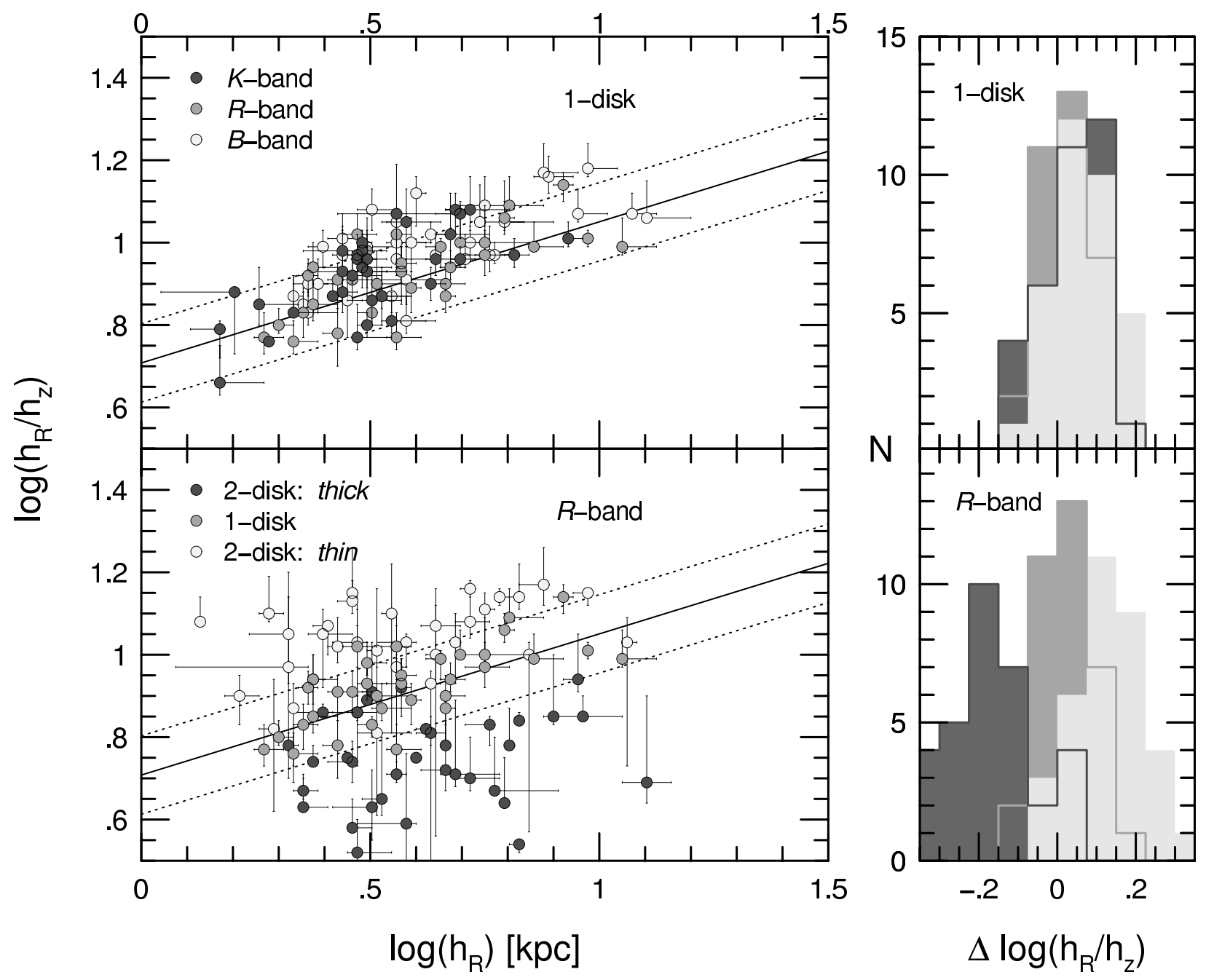

Fig. 2.- Radial to vertical scale-length ratio $\left(h_{R} / h_{z}\right)$ distribution for edge-on spiral galaxies from Yoachim \& Dalcanton (2006). Top panels show the distributions as measured for $B$, $R$, and $K$ bands assuming a single-component disk, as coded in the legend. Bottom panels show the distributions as measured in the $R$ band for a single-component disk and a twocomponent disk (thick and thin), as coded in the legend. Solid and dotted lines are the same as in Figure 1. Right panels are histograms of deviations for all galaxies about the best-fit to the Kregel et al. sample, broken down by band (top) or disk component (bottom). 

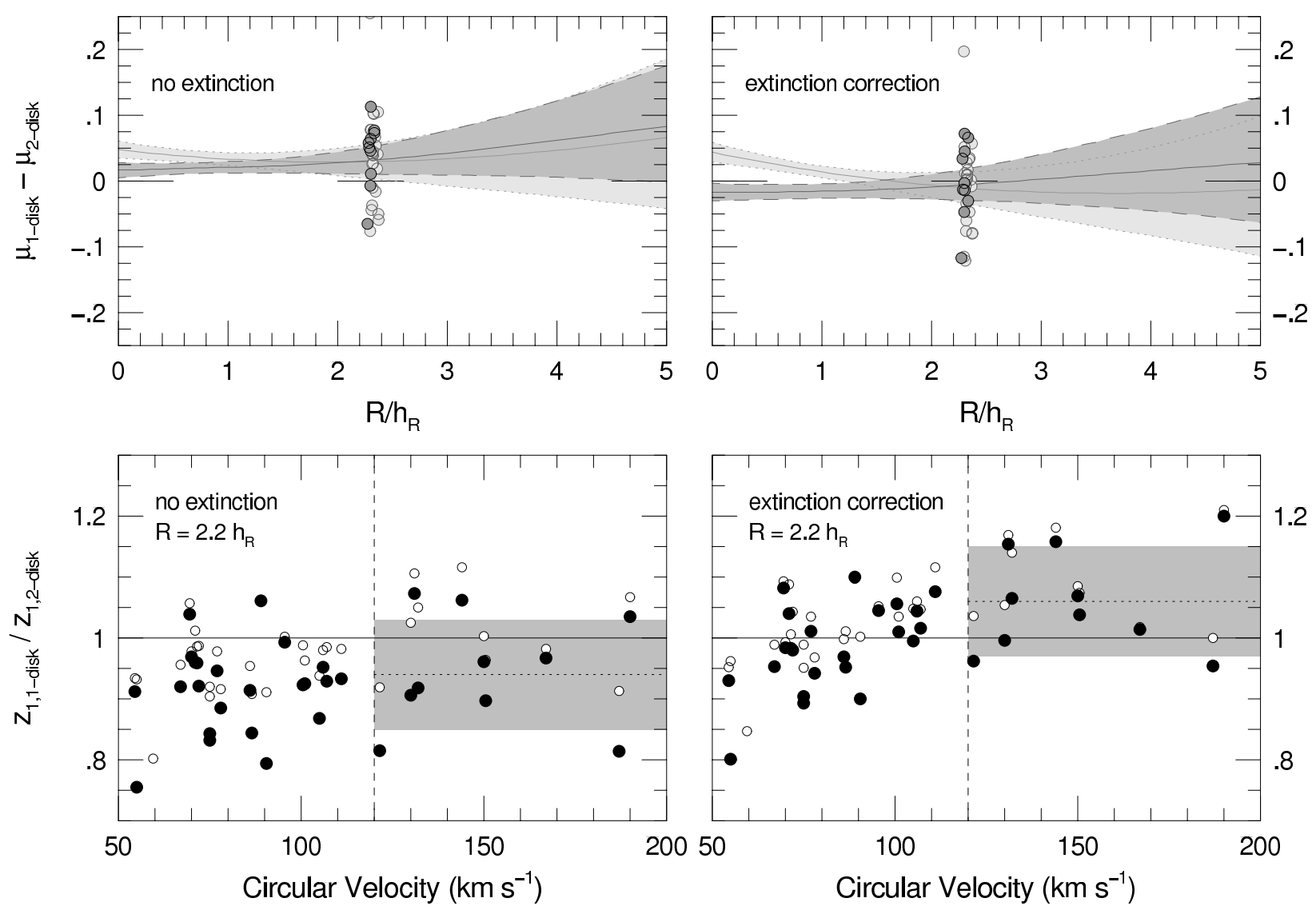

Fig. 3.- Comparison of light-profiles for one and two-component disk fits. Top panels: Face-on surface-brightness difference between one and two-component disk fits from YD, renormalized as described in the text. Dark-grey region represents weighted mean and errors for fast-rotators $\left(V_{\text {rot }}>120 \mathrm{~km} \mathrm{~s}^{-1}\right)$; light grey areas represent the same for slowrotators $\left(V_{\text {rot }}<120 \mathrm{~km} \mathrm{~s}^{-1}\right)$. Individual fits near $\mathrm{R} / \mathrm{h}_{R}=2.2$ are shown as shaded circles; their dispersion is large because of measurement error. Bottom panels: Ratio of the firstmoment of the vertical light-profile for one and two-component models at $\mathrm{R} / \mathrm{h}_{R}=2.2$. Open circles represent moment integrals taken out to $z=6 h_{z}$ of the one-component disk; filled circles represent moment integrals extended to convergence. Gray shaded areas and dotted horizontal lines represent standard deviation and mean for the fast-rotators. Left-hand panels are for fits without correction for extinction. Right-hand panels adopt an extinction model for the thin-disk component, as prescribed by YD. 

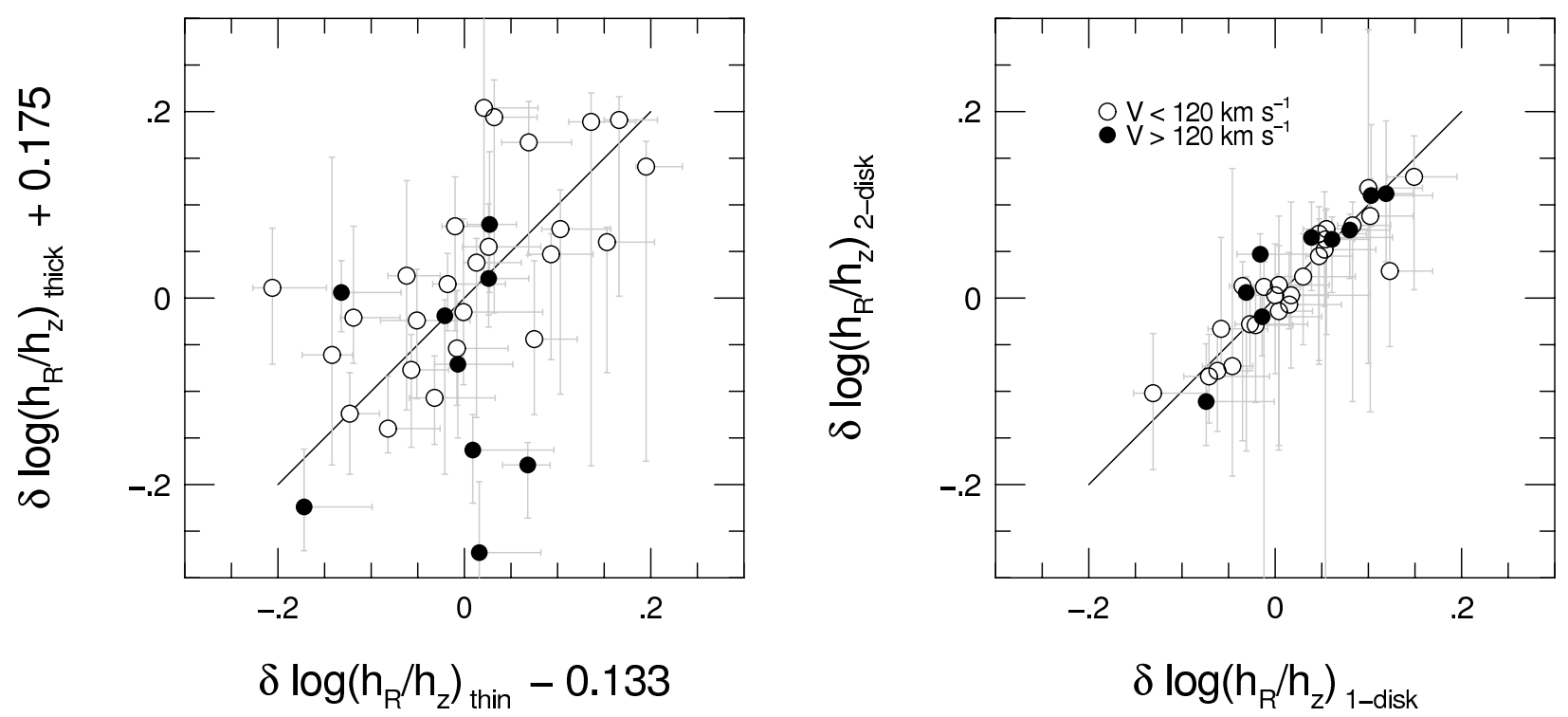

Fig. 4.- Comparison of deviations from mean oblateness-radial scale-length relationship for one and two-component disk fits to the YD sample. The left panel compares thin and thick disk oblateness deviations for the two-component model, where the mean relationship between oblateness and disk radial scale-length is taken to be equation (1) with zeropointadjustments giving zero mean deviation. The right panel compares deviations for the one and two-component disk oblateness, where the two-component value is the light-weighted sum of the deviations for the two components. There is no zeropoint adjustment with respect to equation (1). Solid and open symbols are for fast and slow-rotators, as keyed in the figure. 


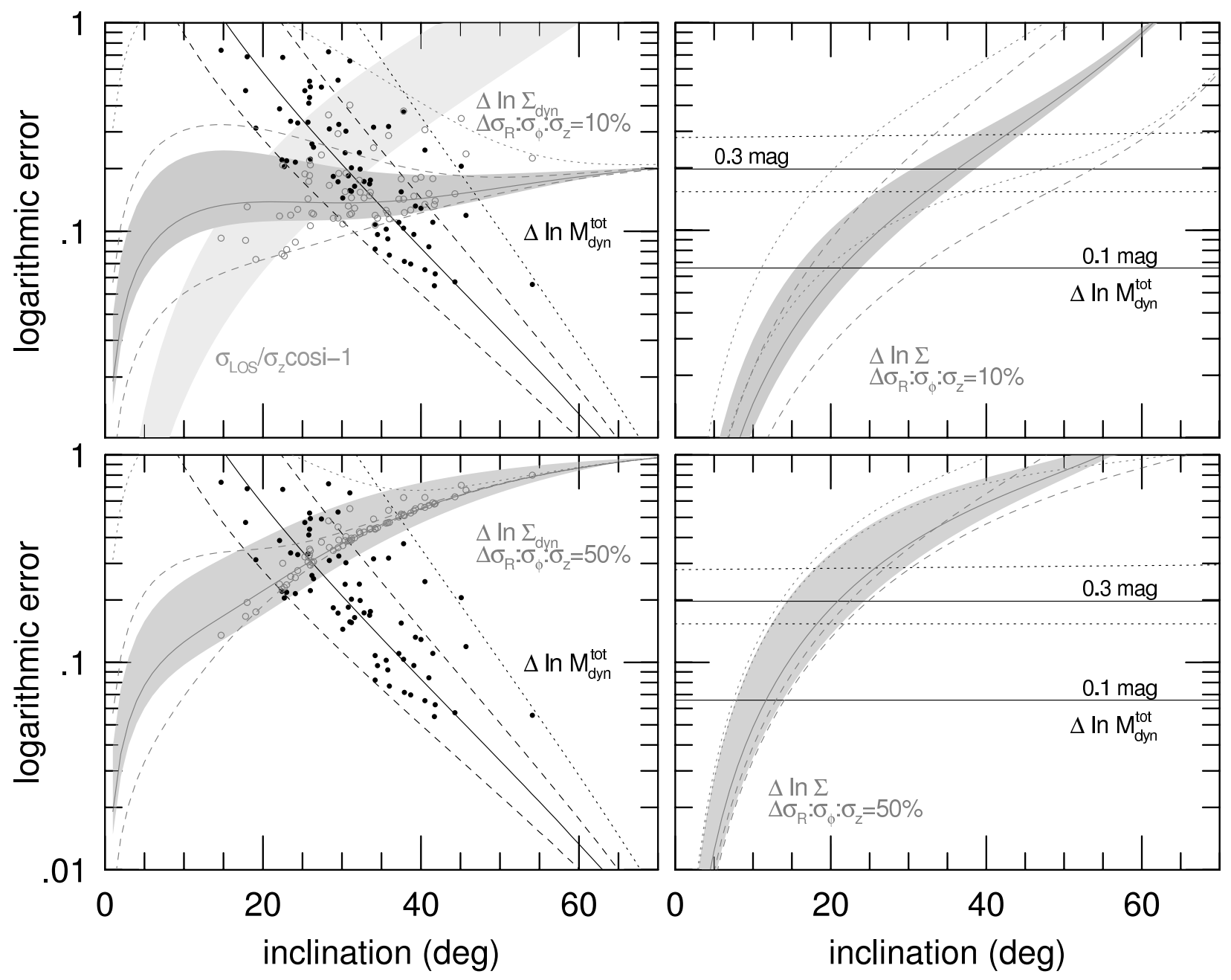

Fig. 5.- Partial DMS error-budget, expressed as logarithmic derivatives, illustrating tradeoffs with inclination and SVE decomposition. Left-hand panels adopt kinematic inclinations and inclination errors; right-hand panels adopt iTF inclinations and errors for a range of slopes and TF-scatter (mag). Top panels assume the SVE shape is known to 10\%; bottom panels assume a $50 \%$ precision. In all panels, black curves, horizontal lines, and filled points represent total-mass errors $\left(\Delta \ln \mathcal{M}_{\text {dyn }}^{\text {tot }}\right)$; dark-gray open points, curves and areas represent disk-mass errors $\left(\Delta \ln \Sigma_{\text {dyn }}\right)$. Light-gray area in top-left panel represents the fraction of the observed velocity dispersion $\left(\sigma_{\mathrm{LOS}}\right)$ to the projected vertical component $\left(\sigma_{z} \cos i\right)$ in a form proportional to the expected systematic error in deriving $\sigma_{z}$ from $\sigma_{\mathrm{LOS}}$. See text for details. 

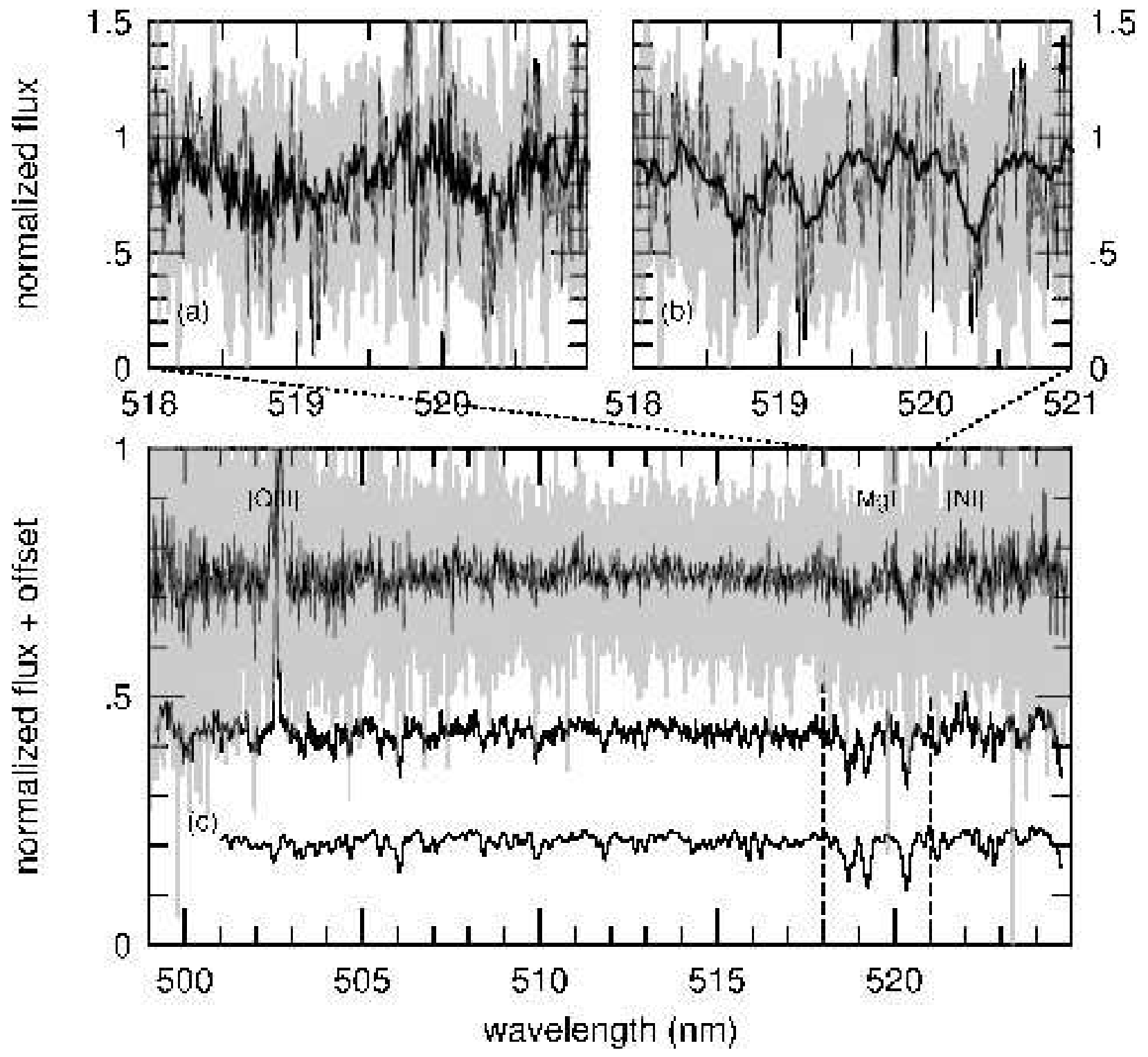

Fig. 6. - Fiber stacking in the Mg Ib-region for SparsePak "ring 4" observations of UGC 6918 (see text) in wavelength space for (a) un-registered spectra and (b) velocity-registered spectra - both over the redshifted window containing only the Mg Ib-triplet. Spectra are continuum-normalized to unity. Gray lines represent individual fiber spectra, with one fiber highlighted by a thin black line; the thick, black line represents the averaged spectrum. The full spectra are shown in (c) from top to bottom: the un-registered spectra and averaged spectrum, the velocity-registered spectrum, and the broadened, K1 III template used to register the galaxy spectra. Spectra are offset in flux for clarity. The location of the $\mathrm{Mg} \mathrm{Ib}-$ triplet absorption and nebular emission from [O III $] \lambda 5007$ and the $[\mathrm{N} \mathrm{I}] \lambda \lambda 5198,5200$ doublet are indicated. Many weak Fe and Ti absoption lines are also visible (see Figure 9). 


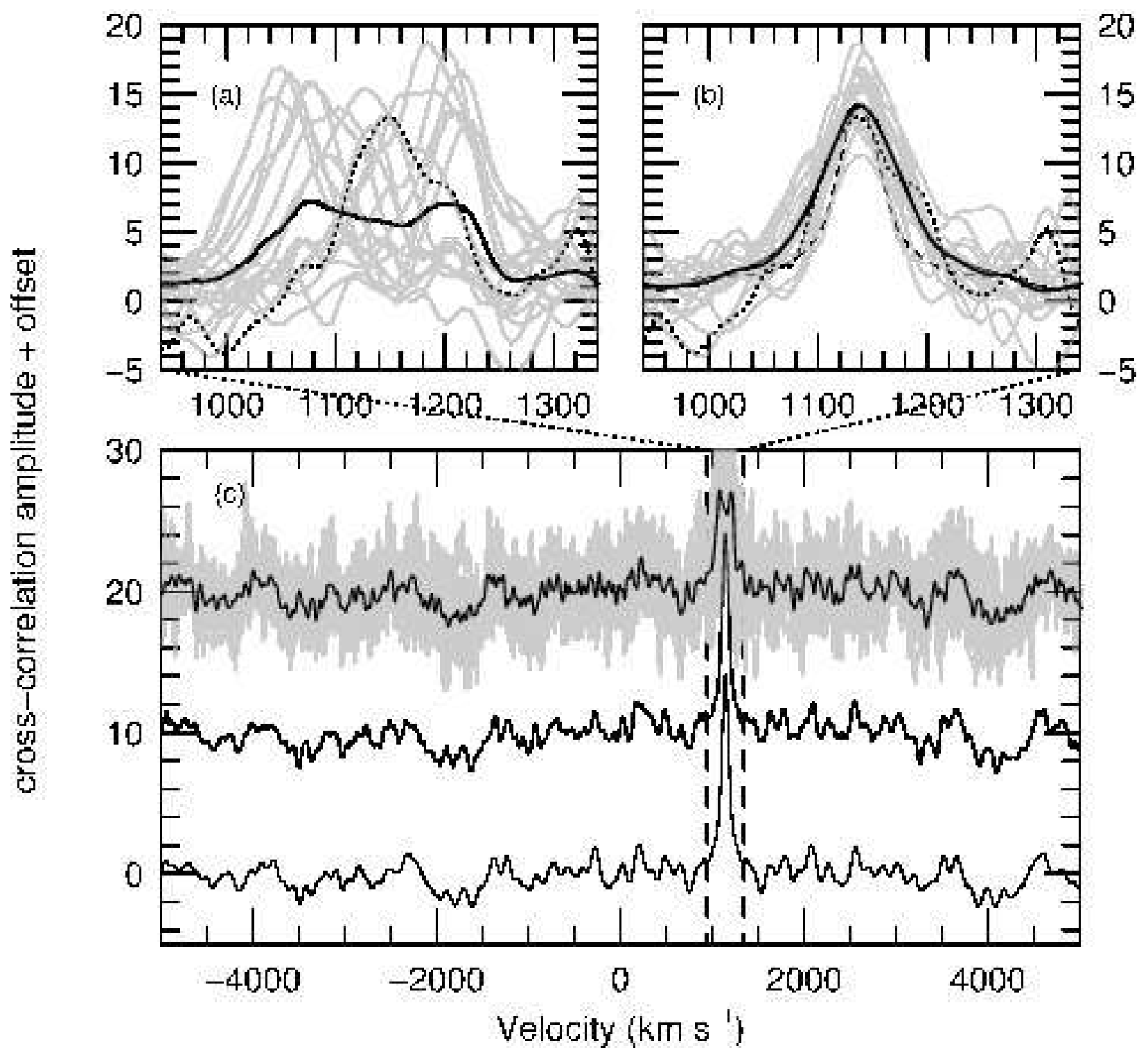

Fig. 7.- Fiber stacking in the Mg Ib-region for SparsePak "ring 4" observations of UGC 6918 in velocity space for (a) un-registered cross-correlations and (b) velocity-registered cross-correlations - both within $\pm 200 \mathrm{~km} \mathrm{~s}^{-1}$ of the galaxy recession velocity. The crosscorrelation template is the un-broadened K1 III star shown broadened in Figure 6. Gray lines represent individual fiber correlations, with one fiber highlighted by a dotted, black line, and the averaged spectrum correlation shown as a thick, solid line. Thin and dashed black lines in (b) represent the broadened-template correlation and the un-broadened template auto-correlation, respectively. Cross-correlations between $\pm 5000 \mathrm{~km} \mathrm{~s}^{-1}$ are shown in (c) from top to bottom: the un-registered spectrum, the velocity-registered spectrum, and the broadened, K1 III template used to register the spectra. Cross-correlations are offset in amplitude in panel (c) for clarity. 


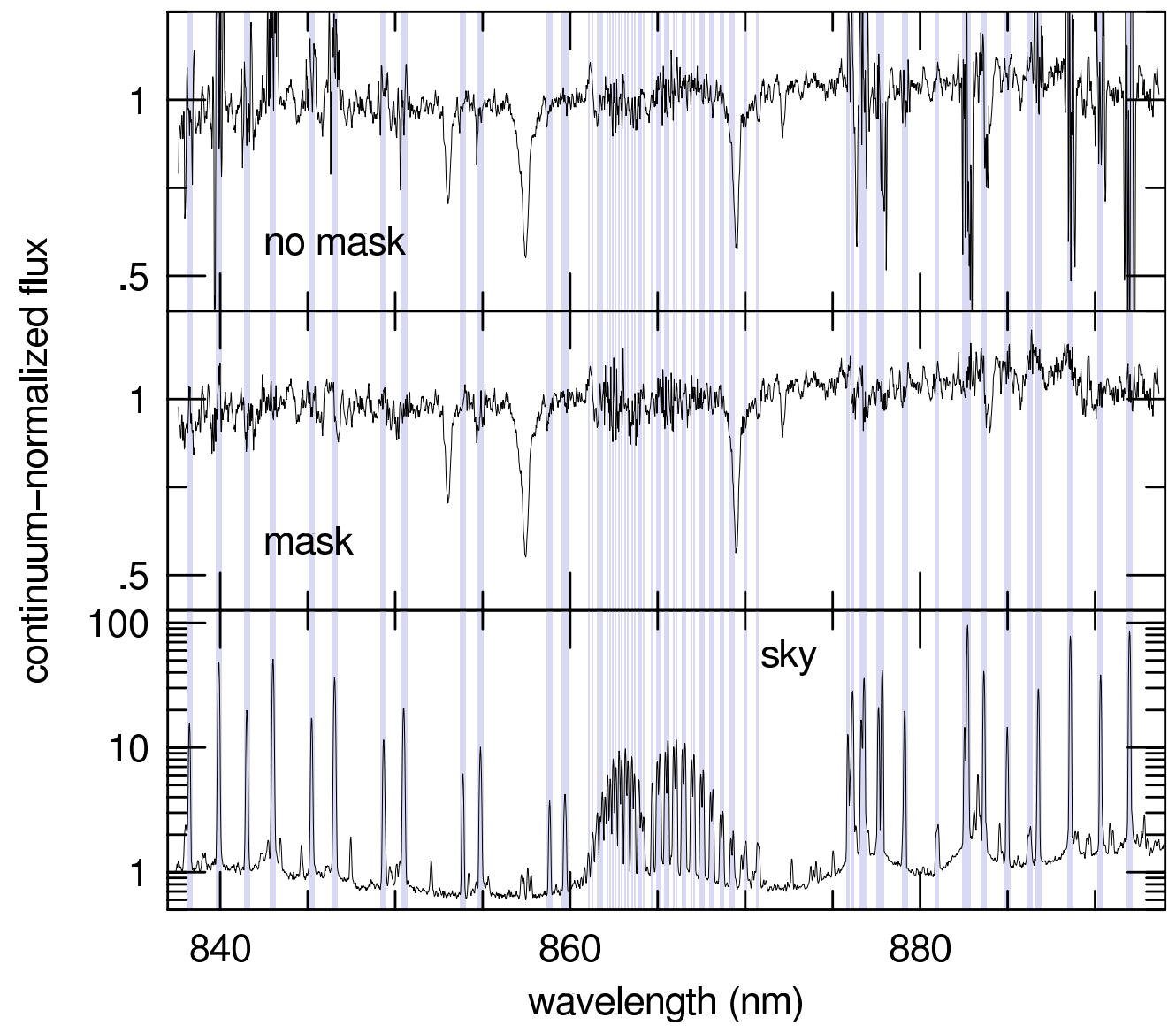

Fig. 8.- Fiber stacking in the Ca II-triplet region for SparsePak "ring 3" observations of UGC 6918, with an internal projected velocity spread of $153 \mathrm{~km} \mathrm{~s}^{-1}$. The top panel shows the velocity-registered co-addition of 12 fiber spectra without masking bright skyline regions. The middle panel has the same registration, but is co-added with masking. The bottom panel shows sky spectrum from the same data. Fluxes are normalized to the mean continuum level; sky-continuum and galaxy continuum at this radius are comparable. Gray-shaded regions indicates masks. Only the brighest lines have been masked (see text). 

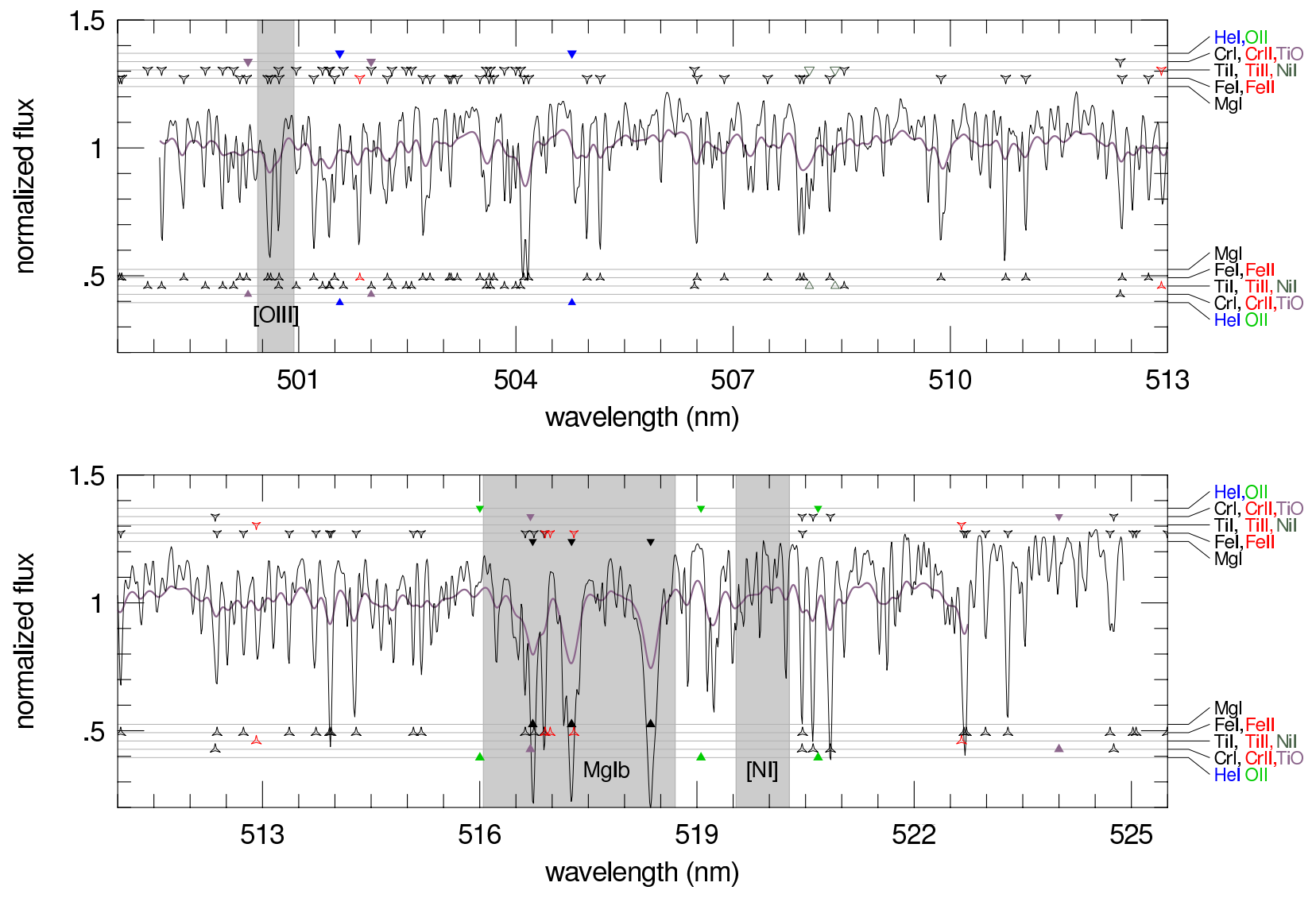

Fig. 9.- HR 6817 (K1 III) template star observed at $\mathrm{R}=11,750\left(\sigma_{\text {inst }}=10.8 \mathrm{~km} \mathrm{~s}^{-1}\right.$, thin black line), and smoothed to the measured broadening of the galaxy spectrum in Figure $6\left(\sigma_{\mathrm{LOS}}=28.9 \mathrm{~km} \mathrm{~s}^{-1}\right.$; thick line). Lines contributing to absorption in this spectrum are marked above and below in four tiers (inside out): Mg Ib triplet (filled triangles); Fe I (black open deltoids) and Fe II (red open deltoids); Ti I (black open deltoids), Ti II (red open deltoids), and Ni I (gray open triangles); Cr I (black open deltoids), Cr II (red open deltoids), and $\mathrm{TiO}$ (gray filled triangles). For reference a last outer tier marks HeI (blue filled triangles) and OII (green filled triangles) absorption features in hot stars. Line identifications are taken from the ILLSS Catalogue (Coluzzi 1993). Vertical shaded regions mark the "Mg Ib" subregion, and the masked regions around [O III] $\lambda 5007$ and $[\mathrm{N} \mathrm{I}] \lambda \lambda 5198,5200$ nebular lines. [COLOR FIGURE FOR ELECTRONIC EDITION ONLY.] 

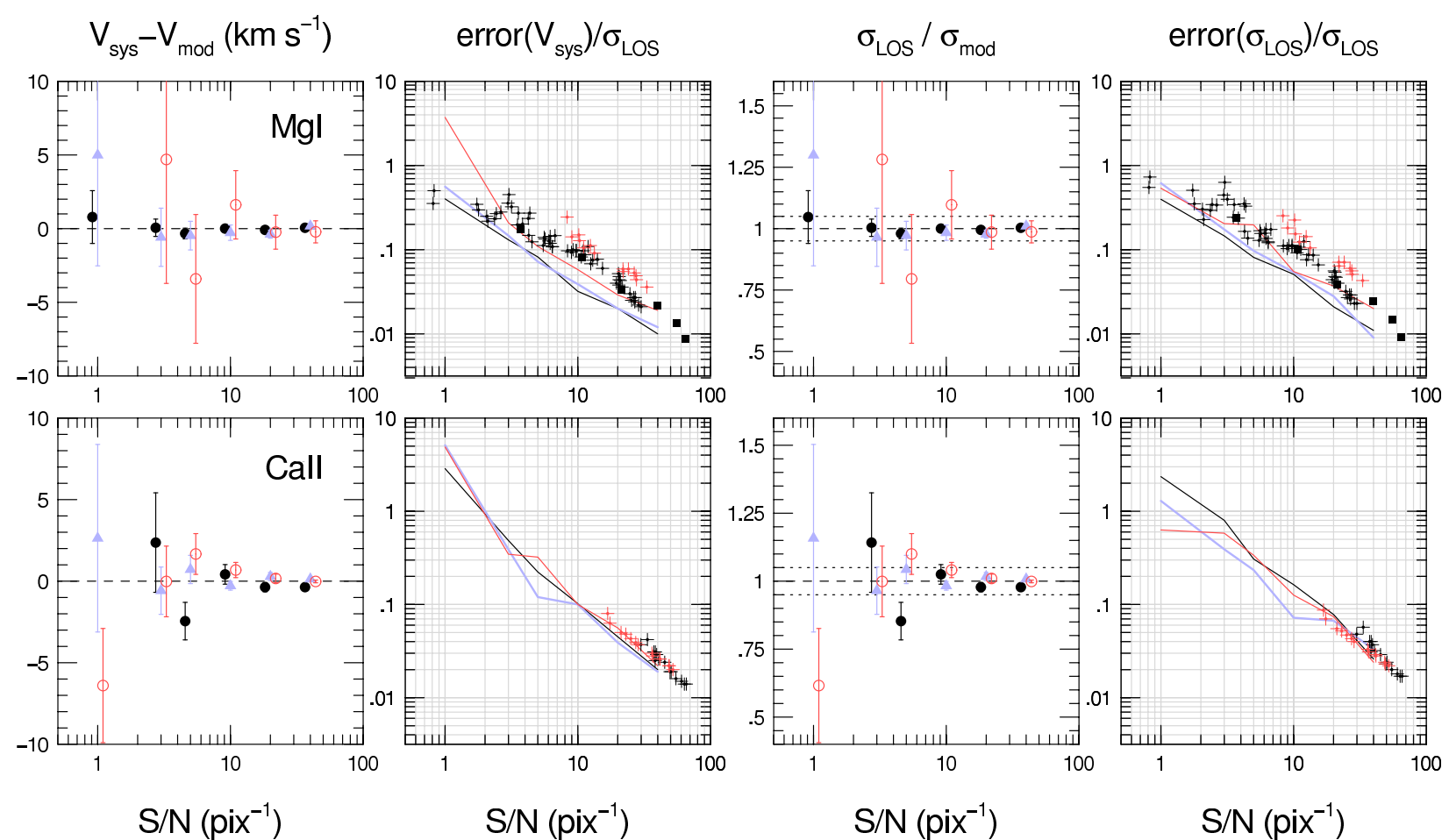

Fig. 10a.- Simulations and measurements of random errors in velocity $\left(V_{\text {sys }}\right)$ and velocity dispersion $\left(\sigma_{\mathrm{LOS}}\right)$ versus spectral-continuum $\mathrm{S} / \mathrm{N}$ (per pixel) in the $\mathrm{Mg}$ Ib region (top row) and Ca II-triplet region (bottom row). Measurements of simulated galaxy spectra (symbols in first and third column from left; lines in second and fourth columns from left), use the same methods applied to observed galaxy spectra, and are referenced to simulation-model values $\left(V_{\text {mod }}, \sigma_{\text {mod }}\right)$. Simulatios use a K1 III template. Colors and symbols indicate $\left(V_{\text {mod }}\right.$, $\left.\sigma_{\text {mod }}\right)$ in $\mathrm{km} \mathrm{s}^{-1}$ : black and filled circles $(1110,20)$; blue or light gray and filled triangles $(1110,60)$; and red or medium gray and open circles $(2350,180)$. The first two cases bracket the observed range for spiral galaxy UGC 6918; the latter characterizes the elliptical UGC 11356. Note the errors in $V_{\text {sys }}$ (second column) are normalized by the measured velocity dispersions, $\sigma_{\mathrm{LOS}}$, as are the errors in $\sigma_{\mathrm{LOS}}$ (fourth column); the latter is therefore equivalent to $\Delta \ln \sigma_{\text {LOS }}$. Horizontal dashed and dotted lines in first and third columns are for reference. Cross-correlation measurements using a K1 III tempate for SparsePak fibers sampling UGC 6918 (black symbols) and UGC 11356 (red or medium gray symbols) are shown in the second and fourth columns. Individual fiber measurements are shown as pluses; stacks of fibers are shown as filled squares. [COLOR FIGURE FOR ELECTRONIC EDITION ONLY.] 

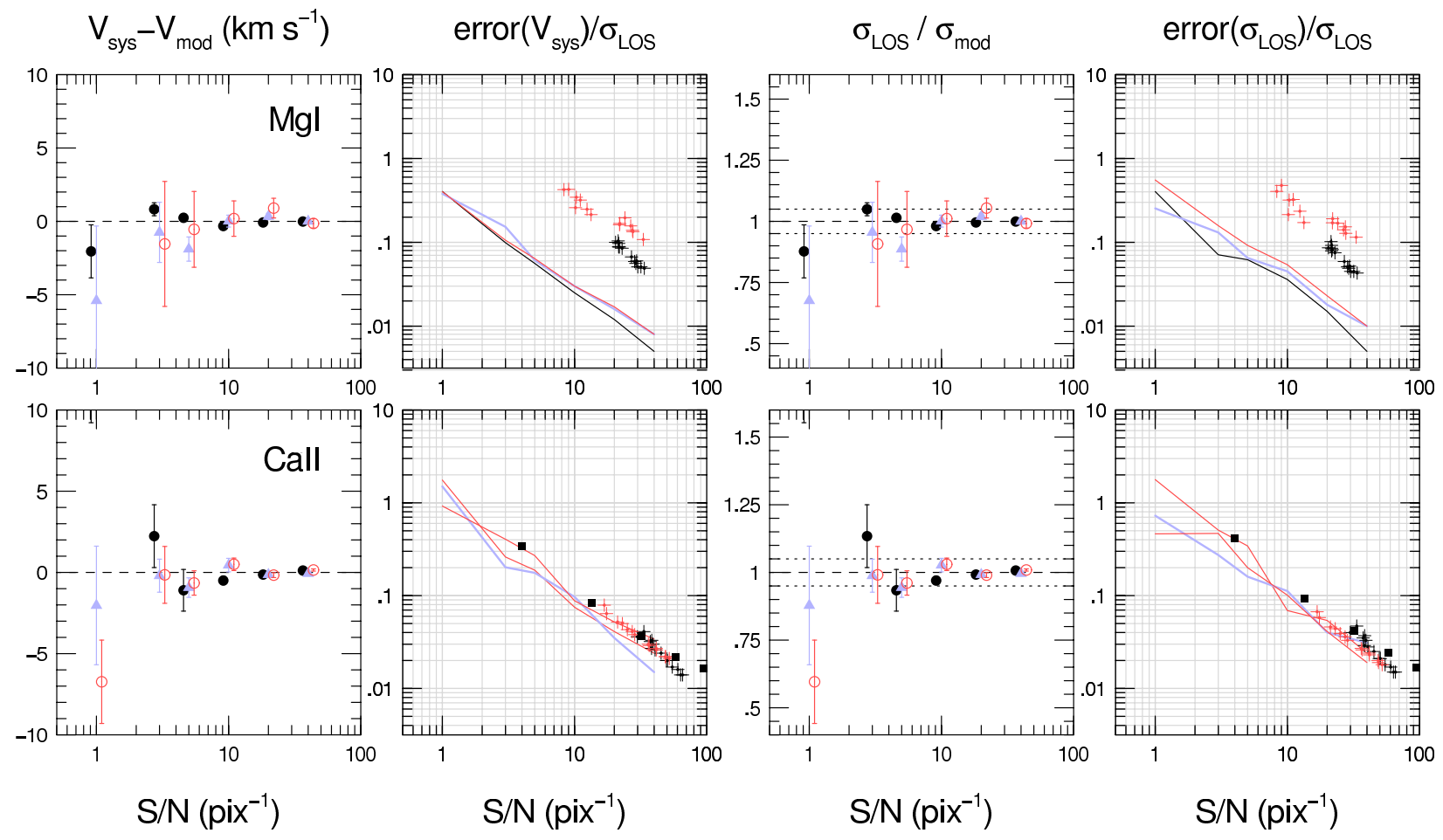

Fig. 10b. - The same as 10a, except using an M3 III template for simulations and crosscorretions with UGC 6918 and UGC 11356. [COLOR FIGURE FOR ELECTRONIC EDITION ONLY.] 


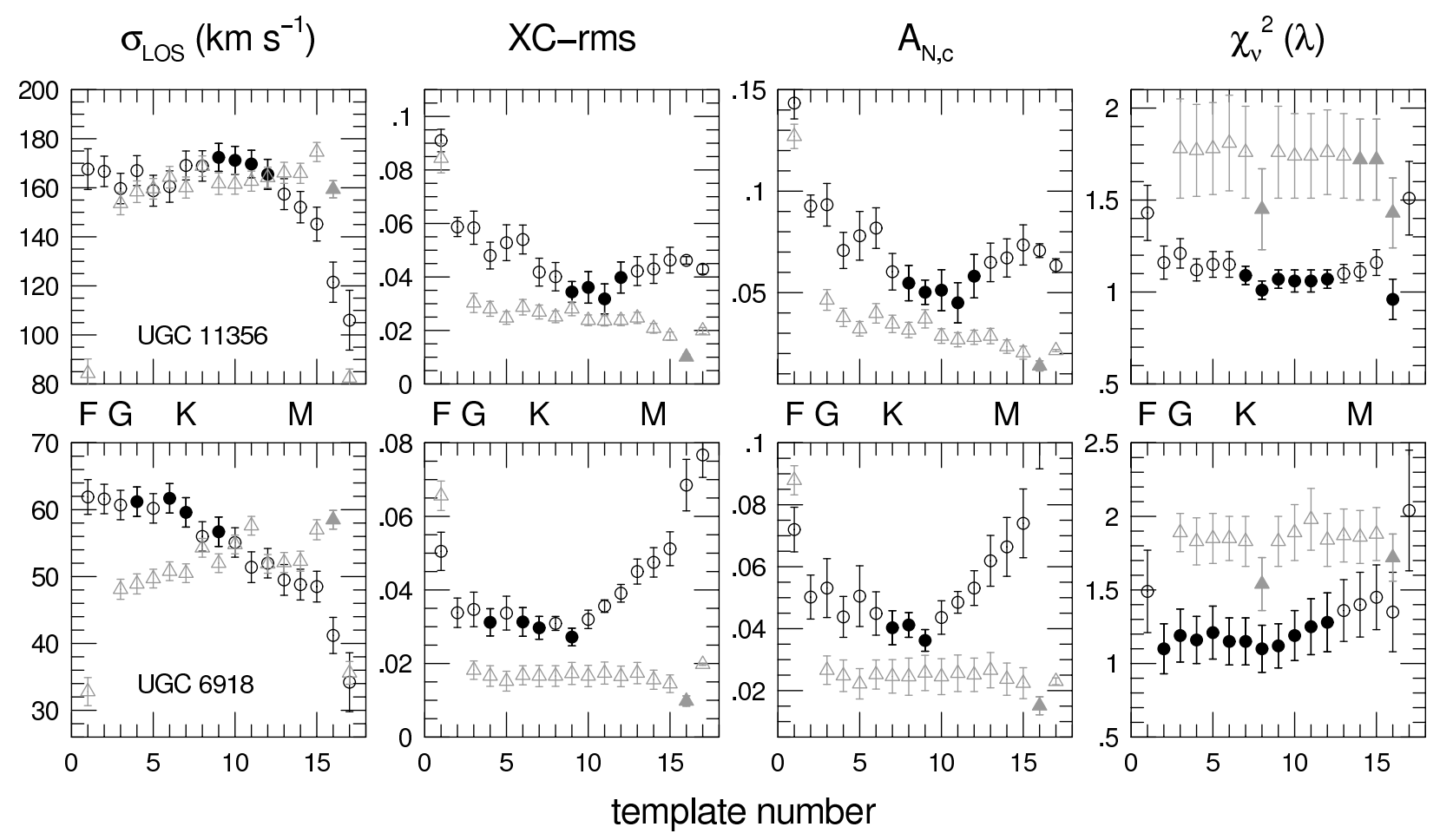

Fig. 11. - Trends of $\sigma_{\mathrm{LOS}}$ (left) and mismatch indices XC-rms, $\mathrm{A}_{\mathrm{N}, \mathrm{c}}$, and $\chi_{\nu}^{2}(\lambda)$ (left to right) with template for the central regions of the elliptical galaxy UGC 11356 (top row) and spiral galaxy UGC 6918 (bottom row). Means and standard deviations are determined in UGC 11356 for 7 fibers between 2 and 6.5 arcsec (effective radius between 19 and 30 arcsec; Bender et al. 1994, Fisher 1997, Gerhard et al. 1998), and in UGC 6918 for 5 fibers between 5 and $10 \operatorname{arcsec}\left(\mathrm{h}_{R}=9.4 \operatorname{arcsec} ;\right.$ Verheijen 1997). Average $\mathrm{S} / \mathrm{N}$ per pixel in the spectral continuum is 25 per fiber in the $\mathrm{Mg}$ Ib region and 45 per fiber in the Ca II-triplet region for both galaxies. Black circles represent Mg Ib-region measurements; gray triangles represent Ca II-triplet region measurements for the same fibers in approximately the same location. Filled symbols in $\sigma_{\mathrm{LOS}}$ and XC-rms columns represent templates that have XC-rms values statistically equivalent to the minimum XC-rms value; in other columns filled circles are keyed in the same way for their respective index. The templates are numbered and ordered by spectral type (giants only, luminosity class II-III), hot to cool, as indicated between the two rows. Templates shown here are near-solar metallicity with the exception of template 8 , (HR 4695, K1 IIIb), which is substantially sub-solar with $[\mathrm{Fe} / \mathrm{H}]=-0.48$. 

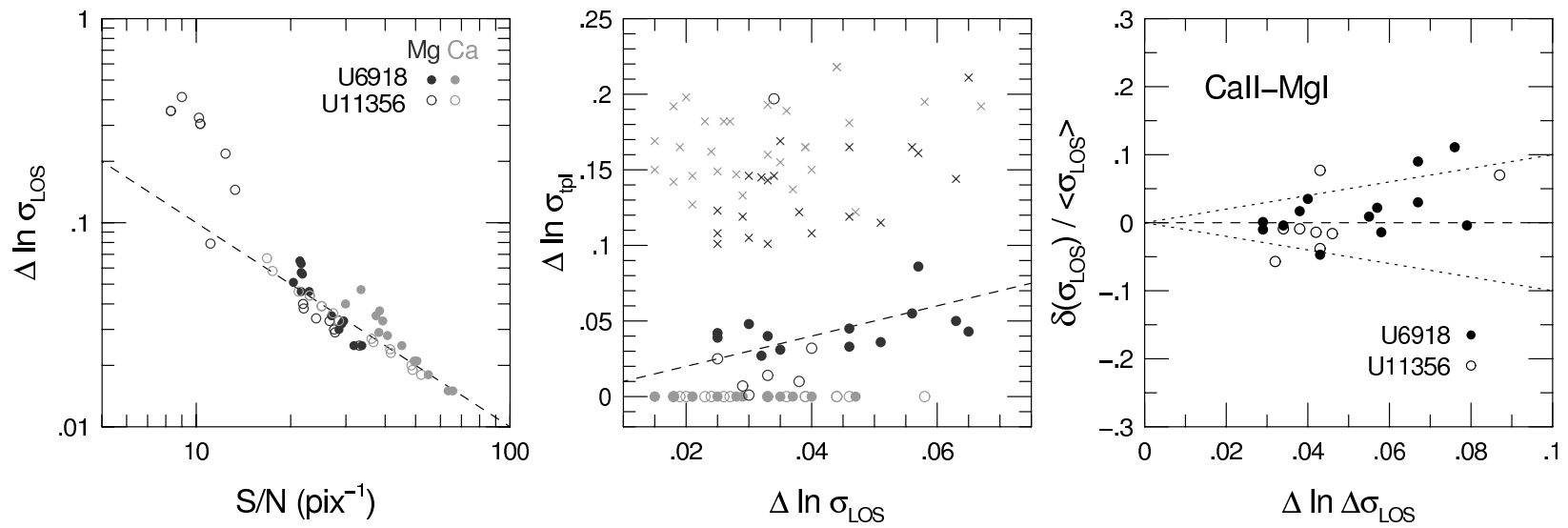

Fig. 12. - Random errors in $\sigma_{\text {LOS }}$ due to shot-noise in the spectra $\left(\Delta \ln \sigma_{\text {LOS }}\right)$ and template mismatch $\left(\Delta \ln \sigma_{\text {tpl }}\right)$ for 14 of the brightest individual fibers in UGC 6918 and UGC 11356 in the $\mathrm{Mg} \mathrm{Ib}$ and $\mathrm{Ca}$ II-triplet spectral regions. The left panel illustrates the dependence of $\Delta \ln \sigma_{\mathrm{LOS}}$ on spectral continuum $\mathrm{S} / \mathrm{N}$; the dashed line depicts the relation $\Delta \ln \sigma_{\mathrm{LOS}}=$ $(S / N)^{-1}$. Symbols are defined in the legend. The middle panel illustrates the amplitude of $\Delta \ln \sigma_{\mathrm{LOS}}$ and $\Delta \ln \sigma_{\mathrm{tpl}}$. Crosses denote systematic errors using our full range of templates (see text); circles denote systematic errors based on limiting templates using the XC-rms index; the dashed line depicts the 1:1 relation. The right panel shows the fractional difference between $\sigma_{\mathrm{LOS}}$ measured in the $\mathrm{Ca}$ II-triplet and $\mathrm{Mg}$ Ib regions using our XC-rms index method $\left[\delta\left(\sigma_{\mathrm{LOS}}\right) /\left\langle\sigma_{\mathrm{LOS}}\right\rangle\right.$, where $\delta\left(\sigma_{\mathrm{LOS}}\right)$ is the difference and $\left\langle\sigma_{\mathrm{LOS}}\right\rangle$ is the mean value for the two regions] versus the random error in this difference $\left[\Delta \ln \delta\left(\sigma_{\mathrm{LOS}}\right)\right]$. Dotted lines illustrate " $1 \sigma$ " boundaries in random error. 

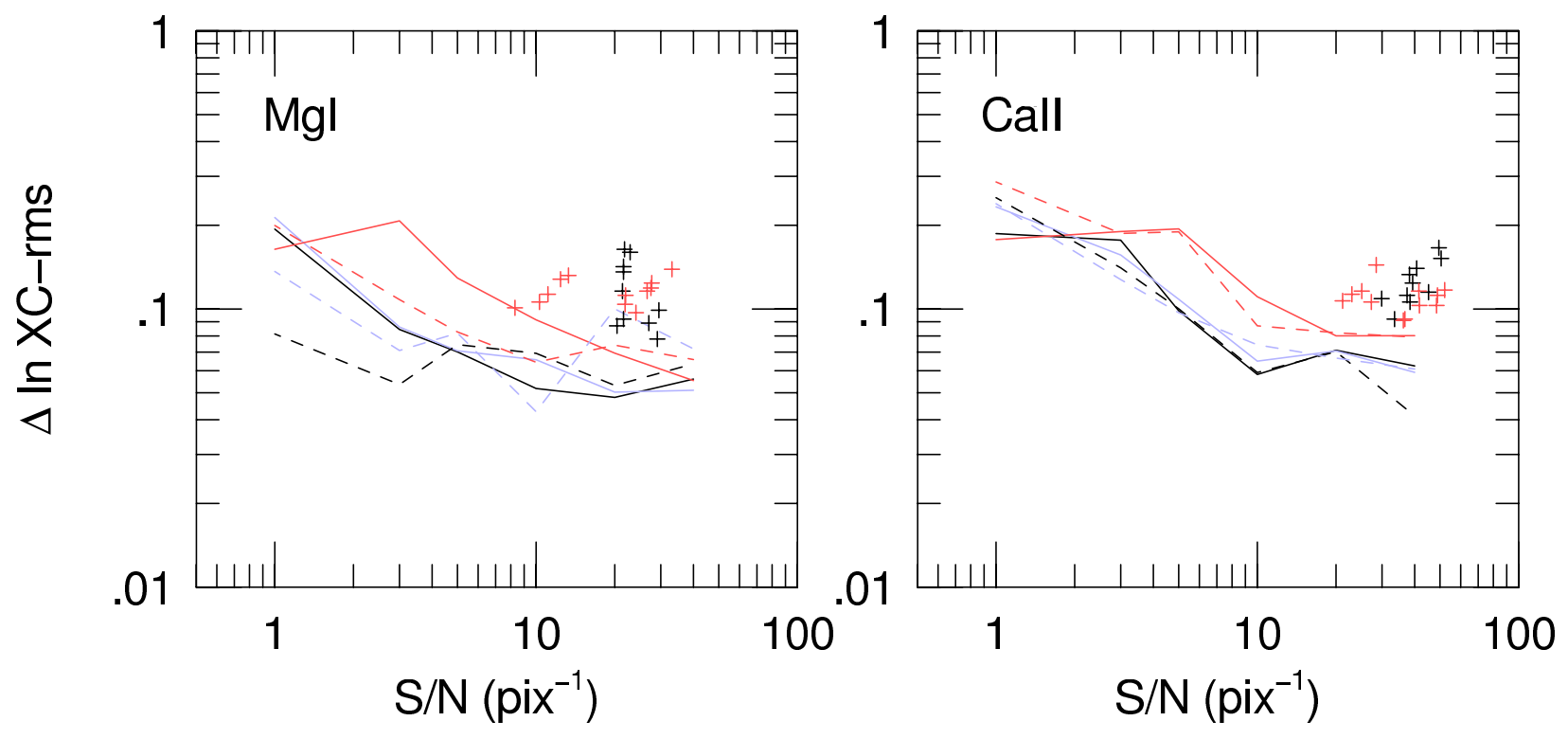

Fig. 13.- Simulations (lines) and measurements (points) of the logarithmic error in the template-mismatch index XC-rms (defined in the text) as a function of spectral continuum $\mathrm{S} / \mathrm{N}$. Lines are simulations using a K1 III template (solid lines) and an M3 III template (dashed lines) color-coded for different broadening as given in Figure 10. Measurements of $\Delta \ln$ XC-rms for individual fibers (plus symbols) use the K1 III template in the MgI-region and the M3 III template in the Ca II-region. UGC 6918 measurements are in blue; UGC 11356 measurements are in red. [COLOR FIGURE FOR ELECTRONIC EDITION ONLY.] 

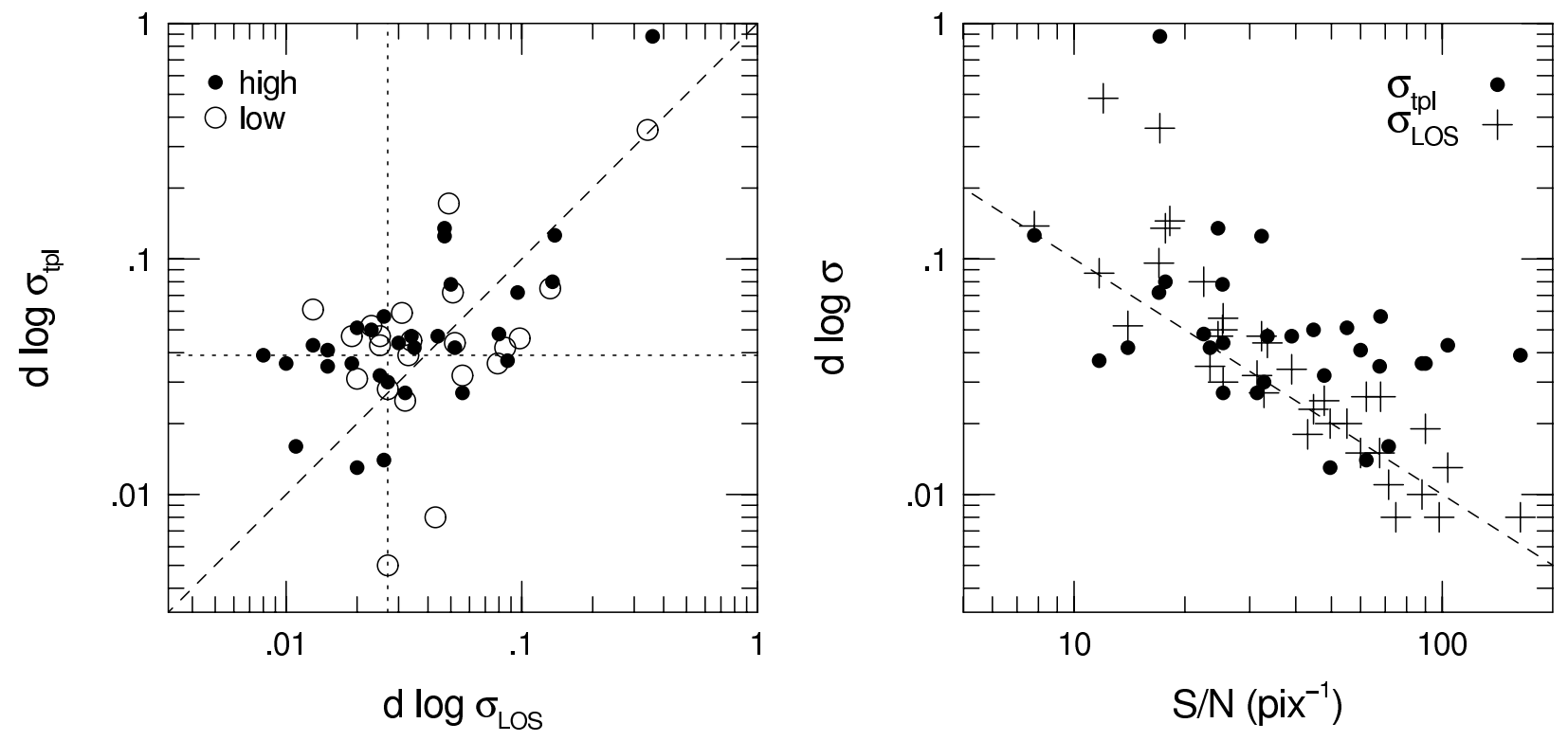

Fig. 14. - Distribution of random errors due to shot-noise in the spectra $\left(\Delta \ln \sigma_{\text {LOS }}\right)$ and template mismatch $\left(\Delta \ln \sigma_{\text {tpl }}\right)$ for azimuthally averaged spectra for 7 spiral galaxies (see text) in our Phase B sample. "High" and "low" identify template-errors assuming $\Delta \ln \mathrm{XC}-\mathrm{rms}$ values of 0.12 and 0.06 , respectively (Figure 13 and text). Horizontal and vetical dotted lines in the left-hand panel are the median values; the dashed line is a 1:1 relation provided for reference. The $\mathrm{S} / \mathrm{N}$ range spanned in the right panel is representative of our survey (Paper I). The diagonal dashed line is the same relation between $\Delta \ln \sigma_{\mathrm{LOS}}$ and $\mathrm{S} / \mathrm{N}$ as adopted in the left panel of Figure 12. 

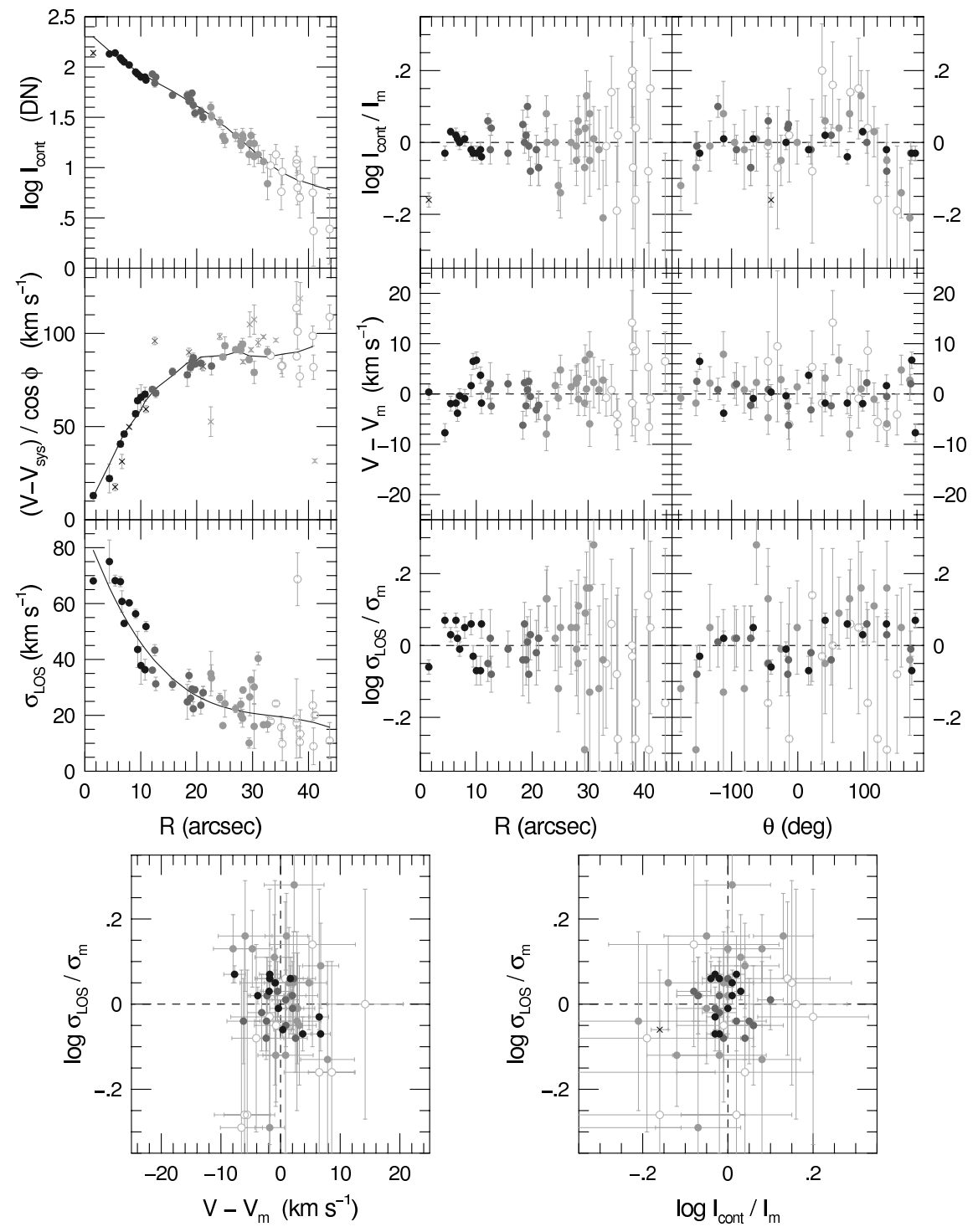

Fig. 15.- Correlations of surface-brightness, velocity and velocity-dispersion residuals: checks on uncorrected systematics for UGC 6918. Top three left-most panels show radial trends of spectral continuum intensity $\left(\mathrm{I}_{\text {cont }}\right)$ in the MgI region, projected velocity $\mathrm{V}$ (deprojected for azimuth but not inclination) and corrected for the estimated systemic recession velocity $\left(V_{\text {sys }}\right)$, and the line-of sight velocity dispersion $\sigma_{\text {LOS }}$. Polynomial fits (curves) define smooth model values $\mathrm{I}_{\mathrm{m}}, \mathrm{V}_{\mathrm{m}}$, and $\sigma_{\mathrm{m}}$ respectively. The inner-most surface-brightness datum is excluded from the fit and marked by $\mathrm{x}$. Velocities are plotted as circles for azimuthal angles within $60^{\circ}$ of the major axis, and x's otherwise. Ratios or differences of observed and model values for all points are shown versus radius $(R)$ and azimuth $(\theta)$ in the galaxy plane in the middle and right top three rows. The model $\sigma_{\mathrm{m}}$ is modulated in azimuth as described in the text. Bottom panels correlate these ratios and differences against each other. Points in all panels represent individual fiber measurements from SparsePak, shaded by radius. 


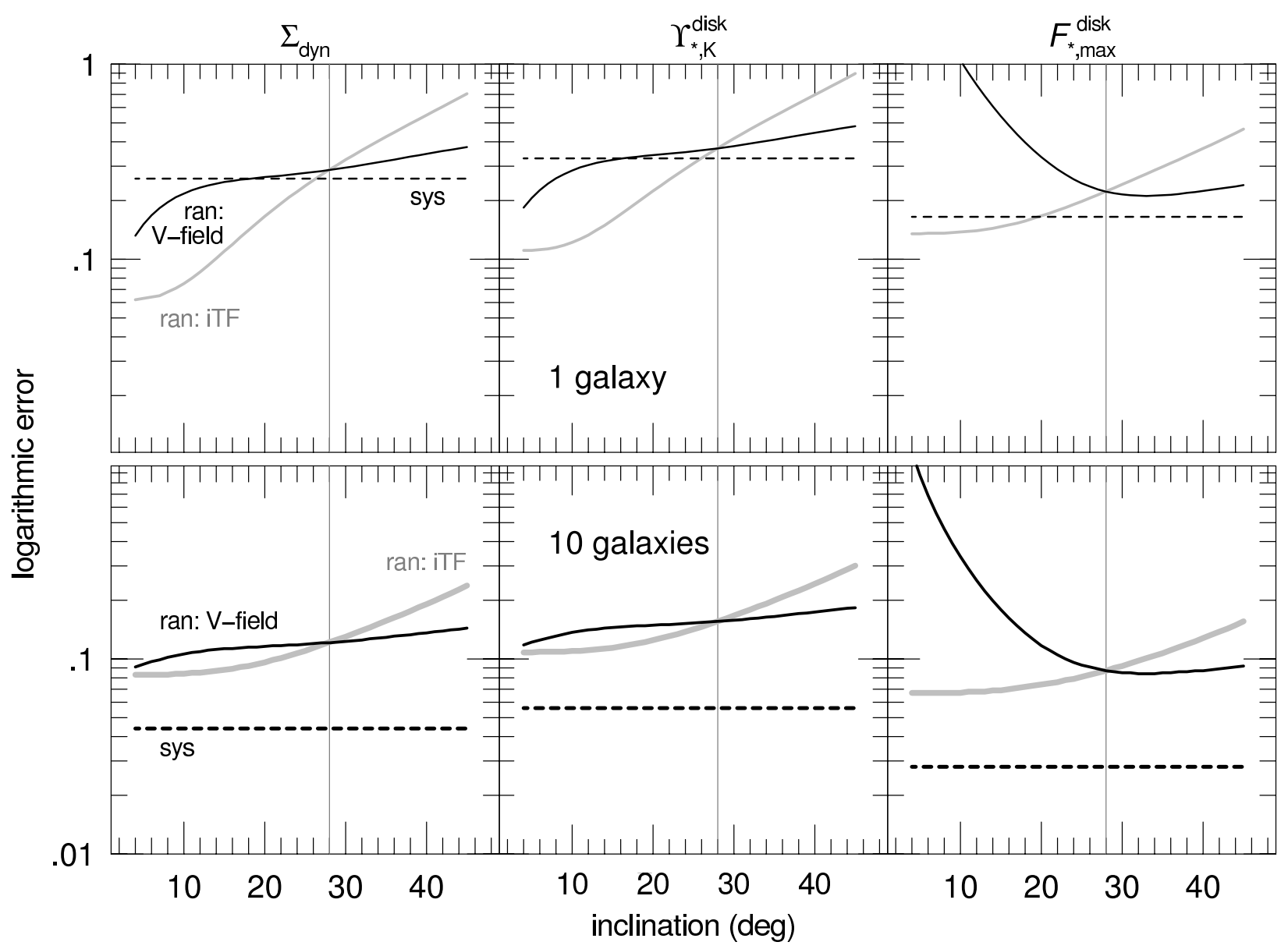

Fig. 16. - Logarithmic errors for $\Sigma_{\text {dyn }}$ (left), $\Upsilon_{*, K}^{\text {disk }}$ (middle), and $\mathcal{F}_{* \text {,max }}^{\text {disk }}$ (right) versus inclination for an single galaxy (top panels) and for averages of 10 galaxies (bottom panels). Systematic errors (sys) are shown as horizontal dashed lines. Random errors (ran) are calculated using estimated inclination errors based on kinematic determinations (black curves), and inverse Tully-Fisher (iTF) determinations (gray curves). The vertical line at $28^{\circ}$ marks the cross-over between these two estimates. 


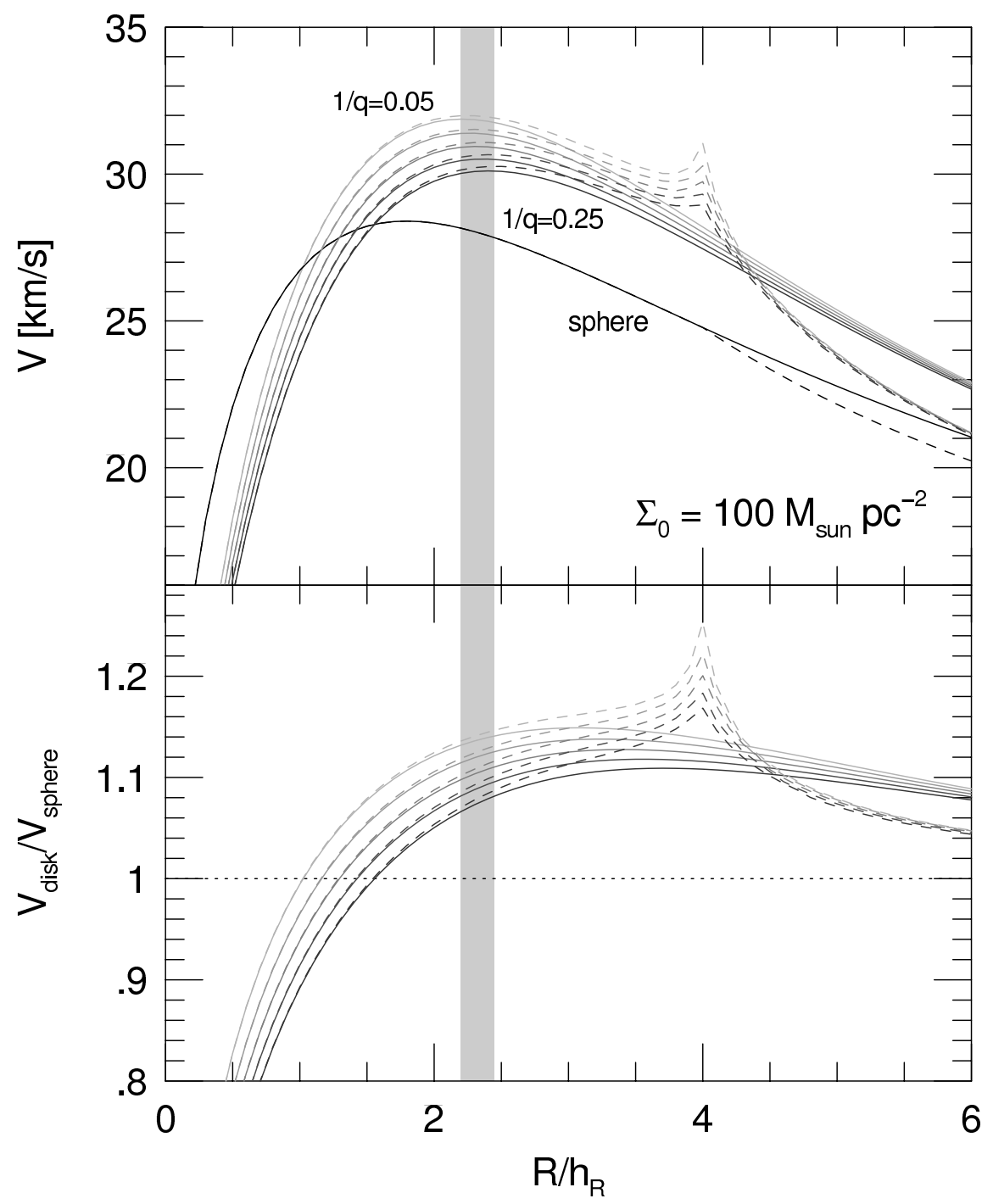

Fig. 17.- Rotation speed of an exponential disk with central mass surface density of 100 $\mathcal{M}_{\odot} \mathrm{pc}^{-2}$ and oblateness $0.05<q<0.25$ versus radius normalized by scale-length, compared to a spherical density distribution with the same enclosed mass. Bottom panel shows the ratio of spherical to disk velocities. Dashed and solid lines show disks truncated at $\mathrm{R} / \mathrm{h}_{R}=4$ and 10, respectively. The radial range where these disks have peak velocities is shaded in gray. 


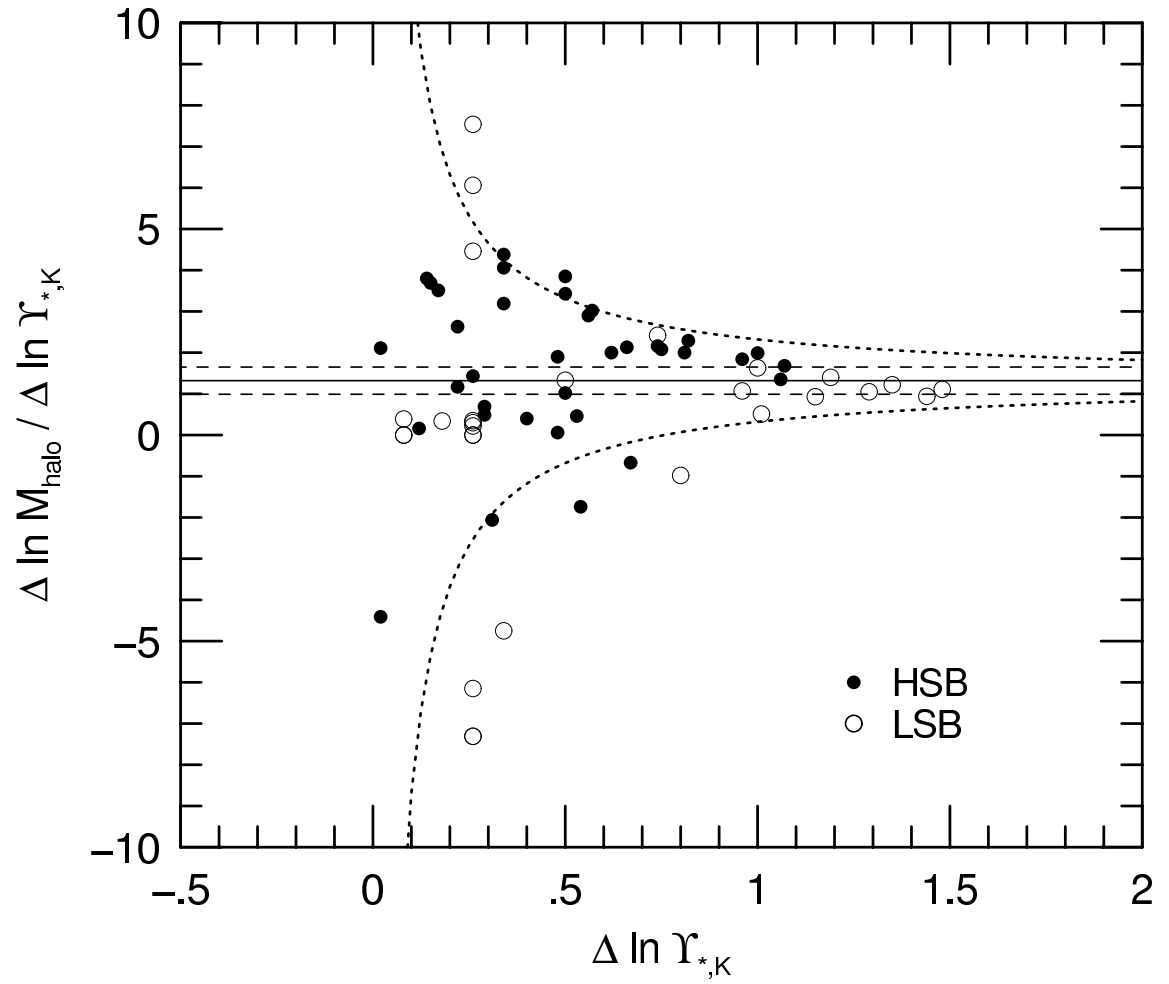

Fig. 18. - Changes in halo mass as a function of stellar mass-to-light ratio for the Ursa Major galaxy sample from Verheijen (1997). Dotted lines illustrate a plausible error-model for this distribution. Solid and dashed lines give the error-weighted mean and error in the mean. Low and high surface-brightness galaxies (LSB and HSB) are marked. 


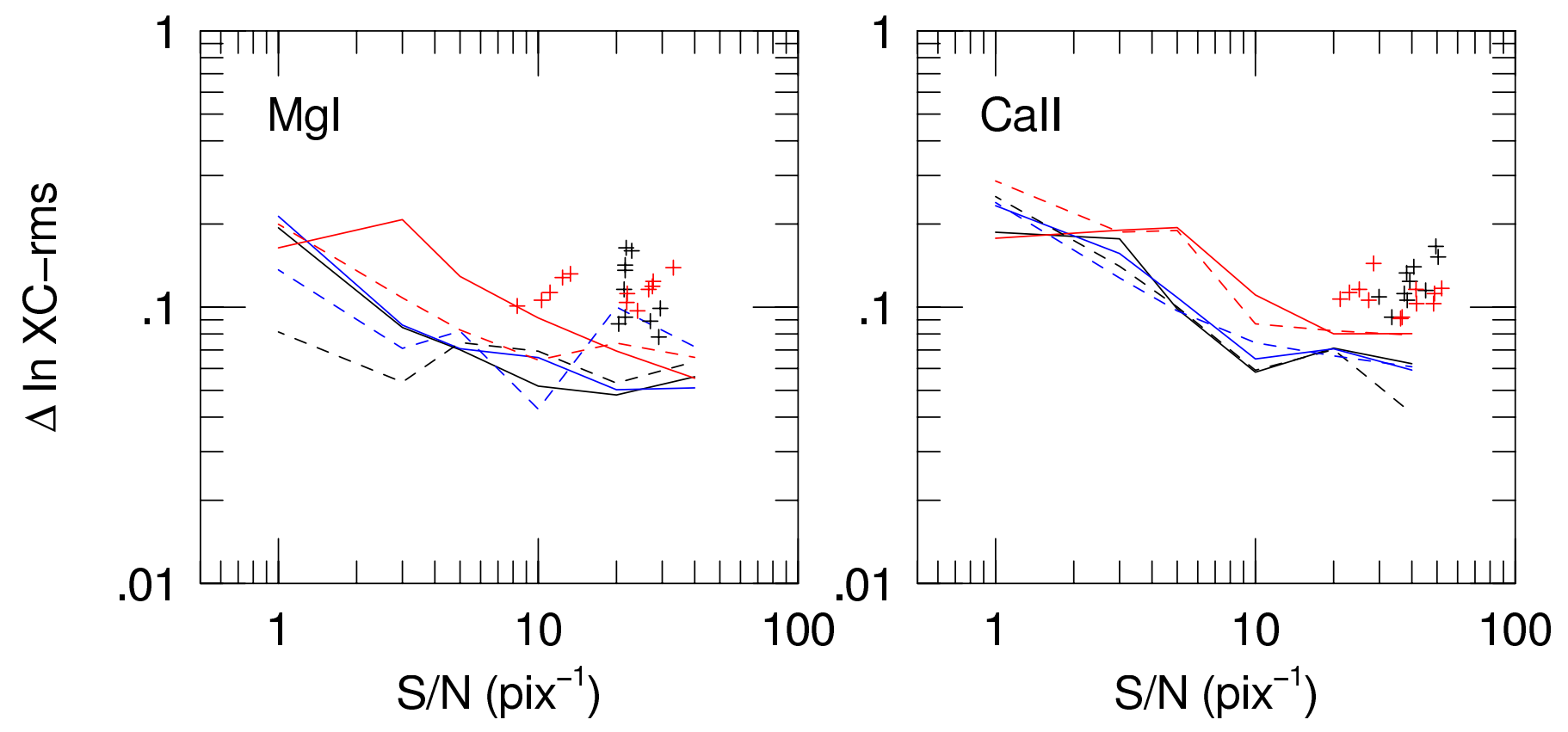

